\title{
Exzerpieren. Eine empirische Studie an Exzerpten von GymnasialschülerInnen der Oberstufe ${ }^{*}$
}

\author{
Gefion Fix/Jürgen Dittmann (Freiburg i. Br.)
}

\begin{abstract}
Taking into account that excerpting texts is a major task for university students of all disciplines, in this study we will investigate pupils' abilities to excerpt texts at the end of their schooling. For that purpose we have analyzed 31 excerpts made by pupils who were currently in their last two years of secondary school. Furthermore we analyzed 33 questionnaires which allowed us to assess pupils' subjective appraisal of the specific excerpting task given to them as well as of their general skills to make excerpts. Most importantly, our findings show that the majority of pupils lack the ability to excerpt texts in a way allowing them to use excerpting as an efficient tool during their university career. It seems that pupils' problems are not primarily rooted in difficulties understanding primary texts but are rather the result of a lack of awareness of what constitutes a good excerpt. We therefore conclude that it is mandatory for universities to teach excerpting texts to their students.
\end{abstract}

\section{$1 \quad$ Einleitung}

Textzusammenfassungen spielen in so gut wie allen Lebensbereichen eine Rolle, so in der privaten Kommunikation, wenn wir Freunden den Inhalt eines Films oder eines Romans erzählen, oder im Betrieb, wenn es gilt, Informationen aus unterschiedlichen Quellen für eine Präsentation aufzubereiten. Von unbestrittener Bedeutung sind Zusammenfassungen auch im wissenschaftlichen Bereich, wo das Abstract einen ersten Zugang zum Inhalt eines Artikels liefert oder der Forschungsstand bezüglich einer Fragestellung in Form kurzer Zusammenfassungen bereits vorliegender Studien referiert wird. Und nicht zuletzt auf dem Feld des "akademischen Schreibens" sind Zusammenfassungen omnipräsent, so bei der Vorbereitung von Referaten, Haus- und Abschlussarbeiten oder von mündlichen Prüfungen und Klausuren. Unter "akademischem Schreiben" verstehen wir demgemäß mit Jakobs (1999: 173) die Formen des Schreibens im Studium, zum Beispiel von Hausarbeiten, die streng genommen noch kein wissenschaftliches Schreiben sind, sondern sich nur an der Normen der wissenschaftlichen Textproduktion orientieren (vgl. auch Gruber et al. 2006: 27f. zum "universitären Schreiben").

Die Forschung hat sich in den vergangenen Jahren denn auch mit Zusammenfassungen beschäftigt: So hat, um einige Beispiele zu nennen, Keseling (1993) Zusammenfassungen im Rahmen der 'prozessorientierten Schreibforschung' behandelt, Endres-Niggemeyer/Schott (1992) untersuchten die Strategien, die erfahrene SchreiberInnen beim Zusammenfassen

\footnotetext{
* Es handelt sich hier um die im Dezember 2007 revidierte und aktualisierte Fassung eines Papiers, das wir im Juli 2004 auf der Homepage von J. D. online gestellt hatten. Aufgrund des vielfältigen Echos auf diese erste Fassung haben wir eine revidierte Fassung für Linguistik online erstellt. Wir danken allen KollegInnen, die sich zur ersten Fassung des Artikels geäußert haben, vor allem aber den beiden anonymen Reviewern für ihre konstruktiven kritischen Hinweise.
} 
anwenden, und Oldenburg (1992) analysierte Zusammenfassungen von Fachtexten, um so die linguistischen Grundlagen für eine effektive Gestaltung des fachbezogenen Fremdsprachenunterrichts zu schaffen. Auch die große Bedeutung von Textzusammenfassungen für Studierende wurde in der Forschung durchaus gesehen (vgl. u. a. Dittmann et al. 2003; Ehlich 1981; Ehlich/Steets 2000; ; Keseling 1993; Kruse/Jakobs 1999). Das studentische Exzerpt "als zusammenfassende Verschriftung eines wissenschaftlichen Textes" thematisiert Moll (2002), im empirischen Teil analysiert sie exemplarisch zwei Exzerpte. Auf diese Arbeit wird im Folgenden mehrfach eingegangen.

Unseres Wissens hat man bislang noch nicht untersucht, wie gut AbiturientInnen bzw. StudienanfängerInnen die für sie so relevante Fähigkeit des Textzusammenfassens/Exzerpierens beherrschen. Die vorliegende Studie entwickelt das Design für eine entsprechende empirische Untersuchung und gibt anhand eines Exzerpt-Korpus' eine erste Antwort auf diese Frage.

\section{Zusammenfassung und Exzerpt - Begriffsbestimmungen und Prozessanalyse}

\subsection{Begriffsbestimmungen}

Die Zusammenfassung ist, mit der Terminologie von Ehlich (1981: 379), als "sekundärer Text" die Reproduktion eines "Primärtextes". Nach Rickheit/Strohner (1989: 220) handelt es sich um einen "Typ von Textreproduktion, bei dem es nicht auf die wörtliche Wiedergabe, sondern auf sinngemäße Wiedergabe des wichtigsten Textinhalts ankommt". Von einer "Textreproduktion" wiederum sprechen die Autoren, "wenn sich die Intention der Textproduktion auf einen früheren ähnlichen Text beziehen lässt" (221). Die Zusammenfassung als sinngemäße Wiedergabe des wichtigsten Inhalts eines Primärtextes unterscheidet sich demnach von solchen Formen der Textreproduktion, bei denen es auf wörtliche Wiedergabe ankommt, wie dem Schreiben nach Diktat, der Rezitation einer Theaterrolle oder dem Erzählen eines Witzes. Sie unterscheidet sich somit auch vom Zitieren, der wörtlichen Wiedergabe von Passagen aus dem Primärtext, das bekanntlich im Bereich des wissenschaftlichen und akademischen Schreibens eine große Rolle spielt.

Zusammenfassungen können sich, wie in der Einleitung angedeutet, auf ganz unterschiedliche Typen von Texten beziehen. So können wir zum Beispiel den Inhalt eines narrativen Textes, z. B. eines Romans, für AdressatInnen, die den Text nicht kennen, oder in einer Prüfungssituation zusammenfassen. Das heißt: wir gewichten den Inhalt im Hinblick auf die Relevanz von Handlungen, Ereignissen und Personenbeschreibungen für das Gesamtgefüge des Textes, produzieren eine Inhaltsangabe. Die einzelnen Handlungen etc. gewinnen bei der Inhaltsangabe ihre Relevanz prinzipiell aus dem Kontext des Primärtextes, was selbstverständlich nicht heißt, dass die Relevanz nicht auch interpretativ strittig sein könnte. Von Bedeutung ist selbstverständlich auch die "Granularisierung" der Inhaltsangabe - ich kann den Inhalt eines Romans prinzipiell in zwei oder auch in 100 Sätzen wiedergeben. Dasselbe gilt z. B. für die Inhaltsangabe eines Spielfilms.

Für Texte, deren Zweck, mit Ehlich (1981: 383) die "Weitervermittlung von Wissen" ist, stellt sich das Problem der Zusammenfassung in anderer Weise. Solche Texte weisen "argumentative Grundstrukturen auf (oder sind auf sie hin orientiert), die die Repräsentierung des Wissens im Text organisieren" (384). Wir werden sie im Folgenden nach Rickheit/Strohner (1989: 237f., vgl. auch Ballstaedt 1999: 110) als "expositorische Texte", im Unterschied zu "narrativen Texten", bezeichnen. Die Zusammenfassung eines Textes des expositorischen Typs, zum Beispiel eines wissenschaftlichen Artikels, setzt mithin, nach Ehlich (1981: 384) "die mentale Rekonstruktion dieser argumentativen Strukturen voraus" (Hervorhebung im Text). "Rekonstruktion" deutet darauf hin, dass die argumentativen Strukturen und damit die 
Gewichtungen der Informationen im Primärtext keineswegs an der Oberfläche des Primärtextes aufscheinen müssen, wie das am ehesten für quasi-standardisierte Darstellungsformen empirischer Beiträge in den Naturwissenschaften oder der Psychologie gelten mag ${ }^{1}$. Sie müssen im Prozess des Textverstehens von den RezipientInnen erschlossen werden, was in der Regel ein gewisses Maß an Übung im Umgang mit den Texten des entsprechenden Wissensgebietes voraussetzt.

Zusammenfassungen von expositorischen Texten muss man also im Kontext von Wissensvermittlung und Wissensverarbeitung sehen, und für diesen Typ von Zusammenfassungen bietet sich die Bezeichnung "Exzerpt" an. Moll (2002: 106) formuliert in diesem Sinne: "Exzerpte werden i. d. R. zu solchen Texten angefertigt, die der Vermittlung von Wissen dienen und daher eine argumentative Grundstruktur aufweisen." Ehlich (1981: 384) charakterisiert das Exzerpieren als "eine Operation zwischen dem Primärtext und weiteren Verwendungen des Wissens, das er enthält" - es leiste "eine Bearbeitung des Primärtextes, durch die die Gewichtung des Wissens im Text erkannt und mentale Anschlussoperationen mit dem im Text verbalisierten Wissen in gewichteter, sortierter Form möglich gemacht werden". Anders als das bloße Zitieren erfordert das Exzerpt ein Reformulieren der Inhalte des Primärtextes und erfordert mithin vor allem "die Fähigkeit des Sich-Distanzierens und des eigenständigen Formulierens" (Steets 1999: 408). Das Exzerpt ist ein "wichtiges Mittel der Wissensaufbereitung", es findet "eine Reduktion von Wissen statt, die der Optimierung des Wissenstransfers zugeordnet ist" (382, Hervorhebung von uns). Bestandteile des so gewonnenen Wissens können dann auch von der Textgrundlage losgelöst in das Langzeitgedächtnis der RezipientInnen überführt werden. Letztlich ist das sogar der normale Gang des Erwerbs "deklarativen" Wissens: Man erinnert sich in der Regel nicht mehr daran, woher man weiß, dass Wasser chemisch $\mathrm{H}_{2} \mathrm{O}$ ist oder "Morphem" die "kleinste bedeutungstragende sprachliche Einheit" meint. Dies im Unterschied zum literarischen Text: Einzelne Ereignisse oder Handlungen haben nur im Kontext des literarischen Werkes (oder im intertextuellen Rahmen etwa der Motivgeschichte) ihren Stellenwert. Wenn ich aus einem literarischen Werk deklaratives Wissen gewinne - also zum Beispiel aus Thomas Manns "Doktor Faustus" lerne, warum Beethovens Klaviersonate op. 111 keinen dritten Satz hat -, dann geschieht dies eben in einem anderen als dem literarischen Rezeptionsmodus.

Die Reduktion hängt also, nach dem, was wir oben über expositorische Texte gesagt haben, zum einen von den Gewichtungen $a b$, die der Primärtext selbst vorgibt und die die RezipientInnen erkennen müssen. Ehlich (1981: 383, Hervorhebung im Text) spricht von dieser Fähigkeit als einer "Kulturtechnik in Gesellschaften, die über komplex organisiertes Wissen erheblichen Umfangs verfügen und gezwungen sind, spezifische Institutionen zur Reproduktion des gesellschaftlichen Gesamtwissens zu unterhalten". Diese Form von "Aktantenwissen" wird von den Mitgliedern der entsprechenden Institutionen verlangt, ohne dass immer klar wäre, wie sie dieses Wissen erlangen können. Wir kommen darauf in Abschnitt 3 zurück.

Doch zielt, wie wir gesehen haben, Exzerpieren auf "mentale Anschlussoperationen mit dem im Text verbalisierten Wissen": Der Erwerb des Wissens ist letztlich auf einen dem Primärtext externen Kontext gerichtet - sei es durch Überführung ins Langzeitgedächtnis, sei es durch Integration in einen selbst verfertigten Text, oder beides. Und deshalb hängt die Reduktion zum anderen von den Gewichtungen ab, die sich aus dem Zweck des Exzerpierens im Prozess des Erkenntnisgewinns für die RezipientInnen ableiten lassen. Wir betonen hier den doppelten Sinn von "Gewichtung": Zum einen die Gewichtung der Aussagen

\footnotetext{
${ }^{1}$ Man denke etwa an die standardisierten "style sheets" von Zeitschriften wie Nature oder Science, die auch die Gliederung von wissenschaftlichen Artikeln vorgeben. Vgl. exemplarisch die Vorgaben von Nature http://www.nature.com/nature/authors/index.html, Stand 02.01.2008.
} 
im Primärtext qua Rekonstruktion des Primärtextsinns, zum anderen die Gewichtung im Hinblick auf den Zweck des Exzerpts (vgl. auch Moll 2002: 107). Es ist sinnvoll, mit Moll (2002: 109) in dieser Hinsicht zwei Typen von Exzerpten zu unterscheiden, wobei diese u. E. die Enden eines Kontinuums denkbarer Übergangsformen markieren:

(1) Exzerpieren für mündliche oder schriftliche Prüfungsvorbereitung: Der Primärtext soll in seiner Gesamtheit komprimiert erfasst werden, damit später ein schneller Zugriff auf die wichtigsten Inhalte möglich ist. In der Regel erfolgt dieses Exzerpieren "nicht unter einer bestimmten Fragestellung, die nur ausgewählte Teilaspekte fokussiert, sondern zielt auf eine verkürzte 'Wiedergabe' des Gesamttextes" (Moll 2002: 109). Diesen Typ von Exzerpt bezeichnet Ehlich (1981: 396) als "objekt-orientiert".

(2) Exzerpieren für Seminararbeiten oder Referate (wir ergänzen: oder Abschlussarbeiten): Hier steht nicht der Argumentationsverlauf des Gesamttextes im Vordergrund, "vielmehr werden in Hinblick auf eine spezifische Fragestellung bestimmte Wissenselemente als relevant erachtet, aus dem Gesamtzusammenhang ausgewählt und entsprechend [...] weiterverarbeitet". Typischerweise werden in Exzerpten dieser Form auch wörtliche Zitate, die später in die eigene Arbeit übernommen werden sollen, aufgenommen (vgl. Moll 2002, 110). Wegen der im Vergleich zu (1) größeren Relevanz der individuellen Gewichtungskriterien nennt Ehlich (1981: 396) diesen Typ von Exzerpt "subjekt-orientiert".

Für die Form des Exzerpts ist schließlich generell von Belang, dass es in der Regel auf die/den VerfasserIn selbst zugeschnitten ist - Ausnahmen sind Exzerpte für eine Arbeitsgruppe oder andere SeminarteilnehmerInnen (vgl. Steets 1999:410) - und deshalb nicht adressatenorientiert formuliert wird: Es spiegelt gleichsam ungebrochen das individuelle Vorwissen und die Gewichtung nach dem jeweiligen Zweck des Exzerpierens, in der Form sind individuelle Gestaltungen möglich, z. B. Abkürzungen, Symbole usw. (vgl. Moll 2002: 108).

\subsection{Prozessanalyse}

Exzerpieren ist, wie jede Form der Textreproduktion, eine komplexe Tätigkeit mit den Komponenten Primärtext, reproduzierter Text (= Exzerpt), ReproduzentIn und mentale Repräsentation des Primärtextes. Beim Reproduzieren wirken sich der Primärtext, die/der ReproduzentIn und die Textrepräsentation auf den reproduzierten Text aus (vgl. Rickheit/ Strohner 1989: 231-236). Damit der Primärtext reproduziert werden kann, muss er zunächst einmal gelesen werden. Das klingt trivial, ist es aber nicht: Die Form des Lesens, um die es hier geht, ist das "Lesen beim Verfassen von Texten", fachsprachlich auch als "Source reading" bezeichnet (vgl. Jakobs 1997: 75). Damit sind Leseprozesse gemeint, die der Einbeziehung von Fachliteratur in die Entwicklung von Positionen und fachlichen Darstellungen dienen" (ebd.) - gerade das ist ja definitionsgemäß beim Exzerpieren der Fall. Genauer geht es um Leseprozesse, "die sich auf andere, bereits vorhandene Texte in ihrer Eigenschaft als Vorlage, Bezugsobjekt oder Informationsquelle für das intendierte Textprodukt beziehen" (Jakobs 1997: 76). ${ }^{2}$ Source reading wiederum wird von unterschiedlichen Faktoren beeinflusst; dazu gehören die individuellen Voraussetzungen der/des RezipientIn (z. B. Motivation und fachliche Expertise, also das Vorwissen der LeserInnen; vgl. Moll 2002: 106f.), die Beschaffenheit des Textes (z. B. die Qualität seiner Strukturierung) und das aktuell zu lösende Problem (vgl. Jakobs 1997: 82f.). Von der Beschaffenheit des Letzteren hängt wiederum ab, "wie gründlich die Quelle konsultiert wird, um welche Inhalte es geht und welche anderen Aktivitäten (Evaluationsprozesse, Elaborationen etc.) das Source reading begleiten bzw.

\footnotetext{
2 Dies im Unterschied zu Leseprozessen, "die sich auf das in Entstehung begriffene Textprodukt selbst richten" (Jakobs 1997 ${ }^{\mathrm{b}}$ :76) - eine Form des Source reading also, die in jeder Form von Textproduktion eine notwendige Voraussetzung der Textrevision darstellt.
} 
ergänzen" (ebd.: 83). An dieser Stelle wird deutlich, dass sich der oben angesprochene Zweck des Exzerpierens als Faktor, der die Primärtext-Reduktion mitdeterminiert, bereits auf das Source reading auswirkt, indem er Evaluationsprozesse steuert.

Wir haben gesehen, dass für die Reproduktion expositorischer Texte die Rekonstruktion der argumentativen Strukturen des Primärtextes entscheidend ist. Nur wenn diese Rekonstruktion gelingt, kann das Exzerpieren als Handlung erfolgreich ausgeführt werden (vgl. Ehlich 1981: 384). Die mentale Repräsentation des Primärtextes als Resultat des Source reading kann man zum Beispiel nach den Modellvorschlägen von Kintsch und van Dijk (vgl. u. a. van Dijk 1980; Kintsch/van Dijk 1983) mittels des Konzepts der Proposition beschreiben, weshalb man auch von "Propositions-Theorie" spricht: Unter einer "Proposition" versteht van Dijk (1980: 44) "eine abstrakte Repräsentierung dessen [...], was wir normalerweise (semantische) Information nennen". ${ }^{3}$ Wir verstehen den Primärtext, indem wir seine Bedeutung im episodischen Gedächtnis als "Textbasis" und als "Makrostruktur" repräsentieren. Textbasis meint hierbei die (an der Oberfläche des Textes orientierte) Folge der Bedeutungseinheiten, der Aussagen oder Propositionen des Textes. Die Makrostruktur dagegen besteht aus einer Menge von Makropropositionen, die die wesentlichen oder globalen Aussagen des Textes wiedergeben und die durch die Anwendung von sog. Makroregeln aus Reihen von (Mikro-)Propositionen des Textes abgeleitet werden. Dies geschieht durch sog. Makroregeln (vgl. van Dijk 1980: 45-49). Eine solche Makroregel ist das "Auslassen": Eine Proposition, die keine Interpretationsbedingung für eine vorhergehende oder nachfolgende Proposition ist, kann getilgt werden. Eine weitere Makrooperation ist das "Generalisieren": Eine Folge von Propositionen kann durch eine allgemeine Proposition ersetzt werden, die beispielsweise den Oberbegriff der Mikropropositionen enthält. Wir werden auf die Makroregeln im empirischen Teil genauer eingehen (vgl. Abschnitt 4.2). Die Anwendbarkeit der Makroregeln wird wiederum durch die "schematische Superstruktur" des Primärtextes mitgesteuert. Darunter versteht van Dijk (1980: 128-139) die globalen Strukturen, die, unabhängig von konkreten Inhalt des Textes, den Typ oder die Textsorte kennzeichnen - also zum Beispiel die Erzählung im Unterschied zum psychologischen Aufsatz oder dem Zeitungsbericht. Die erinnerte mentale Repräsentation eines gelesenen Textes besteht nach dieser Theorie aus der Makrostruktur und einigen Mikrostruktur-Propositionen, die mit den übergeordneten Makrostruktur-Propositionen assoziiert sind.

Nach Kintsch/van Dijk gehen also das Lesen/Source reading bzw. Verstehen eines Textes Hand in Hand mit der Bildung von Makrostrukturen (vgl. Keseling 1993: 5) - und eben diese Makrostrukturen sind per definitionem gewichtet, da sie je nach hierarchischer Ebene die im Text enthaltenen Informationen von jeweils übergeordneten Gesichtspunkten erfassen. Wir kommen darauf in Abschnitt 4.2 zurück. Wenn es zutrifft, dass die Makrostrukturen Resultat solcher Makroregeln wie Auslassen und Generalisieren sind, dann liegen ihrer Bildung offensichtlich Prozeduren zugrunde, wie sie auch für das Schreiben von Zusammenfassungen charakteristisch sind. Man sieht, dass offensichtlich bei der Textreproduktion textrezeptive und textproduktive Prozesse miteinander verflochten sind.

Die repräsentierten Makrostrukturen können dann mit Hilfe unterschiedlicher "Produktionsregeln" in die Zieltextstruktur umgewandelt werden. Diese Produktionsregeln - z. B. lexikalische Substitution und Perspektivenwechsel - sind, anders als die Makrooperatoren, "nicht ohne den (geplanten) Zieltext denkbar" (Keseling 1993: 6), also nicht ohne die konkrete Zielsetzung der Textreproduktion und ohne das relevante Wissen um die Superstruktur eines Zieltextes. In Bezug auf das Exzerpt sei hier auf die Unterscheidung der beiden Typen (vgl.

\footnotetext{
${ }^{3}$ Die Propositions-Theorie und andere Theorien der Textrezeption und -reproduktion diskutieren Rickheit/Strohner (1989: 222-231); zur Theorie von Kintsch/van Dijk vgl. auch Keseling (1993: 3-7).
} 
Abschnitt 2.1) verwiesen, die je unterschiedliche Umsetzungen in den Zieltext zur Folge haben.

Die Komplexität der Prozesse beim Textreproduzieren führt, wie zu erwarten ist, zu großen Unterschieden in der Performanz zwischen erfahrenen und weniger erfahrenen SchreiberInnen (in der Schreibforschung gerne als "ExpertInnen" und "NovizInnen" bezeichnet). ${ }^{4}$ Keseling (1993: 12-15) gibt eine Überblick über die wichtigsten Ergebnisse einschlägiger empirischer Untersuchungen. ExpertInnen gehen etwa folgendermaßen vor: Sie überfliegen den Primärtext zunächst ohne Stichworte zu notieren, um sich ein grobes Bild vom Inhalt zu machen. In einer "postreading/prewriting"-Phase lesen sie den Primärtext noch einmal durch, suchen gezielt nach den zu verwendenden Informationen, machen Notizen und planen auf diese Weise den Zieltext. In der Niederschriftphase unterbrechen sie ihren Schreibfluss des öfteren (ca. doppelt so oft wie NovizInnen!), um nochmals im Primärtext und den Notizen zu lesen. ExpertInnen können besser als NovizInnen zwischen wichtigeren und unwichtigeren Informationen unterscheiden - sie produzieren also eher korrekte und effiziente Makrostrukturen und hierarchisieren sie angemessener, wozu auch gehört, dass sie Informationen aus unterschiedlichen Abschnitten des Primärtextes rekombinieren können.

\section{$3 \quad$ Die Bedeutung des Exzerpierens für Studierende}

Eine zentrale Aufgabe, die Studierende aller Fachrichtungen an den Hochschulen zu bewältigen haben, ist die Verwertung und Weiterverarbeitung des Wissens, das in der jeweiligen Forschungsliteratur "steckt". Rienecker (1999: 96) behauptet mit Recht: "Knowledge transformation is the hall-mark of what is requested from our students." Und hier hat das Exzerpt seinen Ort. Mit Steets (1999: 408): "Überall dort, wo in kurzer Zeit viel Wissen zur Kenntnis genommen und individuell verarbeitet werden muss, hat das Exzerpt seine besondere Wichtigkeit." Anders gesagt: Das Exzerpt ist ein Mittel, Fachwissen auf dem Wege der Textreproduktion in individuelles Wissen der/des Lernenden zu überführen (vgl. Ehlich 1981: 381). Gerade dies ist aber essenziell für das akademische Schreiben: Fachliteratur muss im Hinblick auf ein bestimmtes Thema - z. B. einer Hausarbeit oder einer Abschlussarbeit - kritisch wiedergegeben werden bzw. rezipierte Texte müssen reformuliert werden. Dabei ist die Fähigkeit zur Herstellung von Text-Text-Bezügen wichtig, da Intertextualität ein konstituierendes Element wissenschaftlicher Texte schlechthin ist (vgl. Jakobs 1997: 83). Das Exzerpieren relevanter Texte vor dem Schreibprozess ermöglicht den gezielten, zeitsparenden Rückgriff auf Primärtexte: Im Gelingensfall enthält das Exzerpt selbst die Informationen, an welcher Stelle der entstehenden Arbeit die in ihm enthaltenen Wissensbestände einzufügen sind, und es ermöglicht die stärkere Konzentration auf den entstehenden Text, weil es, wiederum im Gelingensfall, den Rückgriff auf den - längeren, komplexeren usw. - Primärtext überflüssig macht. Letzteres gilt selbstverständlich auch für Exzerpte zur Prüfungsvorbereitung.

Angesichts der Relevanz, die dem Exzerpieren für das akademische Schreiben zukommt, kann man vermuten, dass Studierende mit wenig entwickelter Fähigkeit des Exzerpierens Probleme mit der Anfertigung von Hausarbeiten haben werden. Dabei sei eingeräumt, dass das Exzerpieren selbstverständlich nicht die einzige Anforderung an Studierende ist, die akademisch schreiben müssen. Probleme kann es deshalb an jeder erdenklichen Stelle des Schreibprozesses geben (vgl. Dittmann et al. 2003: 159-164, für eine Problemanalyse). Umgekehrt ist es denkbar, dass ExpertInnen Text-Text-Bezüge ohne ausformuliertes Exzerpt

\footnotetext{
${ }^{4}$ Vgl. auch Endres-Niggemeyer/Schott (1992) zur professionellen Erstellung von 'Abstracts'. Eine kontrstive Analyse wissenschaftlicher Abstracts findet man in Baßler (2007). Ein differenziertes Prozessmodell für das Zusammenfassen von Texten, das die Komplexität dieser Tätigkeit widerspiegelt, wird in Keseling (1993: 85105) vorgestellt.
} 
- sozusagen auf der Grundlage eines mentalen Exzerpts - realisieren können. Doch fand Keseling (1997: 228) mittels der Befragung von KlientInnen der Marburger Schreibberatung heraus, dass der Störungstyp "Schwierigkeiten bei der Konzeptbildung und beim Abarbeiten von Konzepten" in "etlichen" Fällen mit Problemen beim "Zusammenfassen von Texten" einherging. Diese Probleme beschreibt Keseling (ebd.) wie folgt: "Statt einen Text in eigenen Worten zusammenzufassen, wird unterstriche, 'herausgeschrieben', oder es werden Karteikarten angelegt. In anderen Fällen werden zwar Zusammenfassungen geschrieben, diese werden aber viel zu lang, enthalten auch Unwichtiges, wie die Autoren oft selbst bemerken, und manchmal bestehen sie hauptsächlich aus Zitaten. Bei der vorausgehenden Lektüre stellt sich der Leser nicht oft genug die Frage nach dem Wichtigen bzw. worum es überhaupt geht." Die TextproduzentInnen gewinnen nicht genügend Abstand vom Text, lösen sich nicht vom Primärtext - sei es aus Unsicherheit, sei es, weil sie gar nicht auf die Idee kommen -, und kürzen den Text nur, vermeiden aber "Generalisierungen, Substitutionen und umorganisierende Operationen" (Keseling 1997: 231f., mit Bezug auf van Dijk/Kintsch 1983). Operationen dieser Art - auf die wir im Einzelnen in Abschnitt 4.2 eingehen - sind aber konstitutiv für jede Art von Textproduktion, denn "ob nun aus mehreren Primärtexten ein neuer Text 'zusammengeschrieben' wird, ob Forschungsergebnisse dargestellt werden, ob eine Argumentation entwickelt wird oder ob aus Stichworten ein endgültiger Text konzipiert wird, stets erfordert dies, dass sich der Autor von vorgegebenen Formulierungen und/oder Schemata löst, eigene Schemata konstruiert und diese dann für eigenen Formulierungen nutzt. Mit anderen Worten: Alte Texte lassen sich nicht direkt in neue umwandeln, sondern nur über mentale Zwischenstationen." (Keseling 1997: 232) Diese Auffassung impliziert, dass das Üben von Textzusammenfassungen immer auch ein Üben von Textproduktion allgemein und speziell der planvollen Konzeptbildung - bedeutet.

Auch Furchner et al. (1999: 43) stellen einen Zusammenhang zwischen Schreibschwierigkeiten im Studium und dem Exzerpieren her. Sie berichten von dem Problem, dass es Studierenden oft nicht bewusst sei, "was im einzelnen dazugehört, eine größere wissenschaftliche Arbeit zu schreiben". Dies könne bedeuten, dass nur gelesen werde, allenfalls Stichworte notiert, aber keine Exzerpte in einer solchen Form angelegt würden, dass sie später auch direkt verwendet werden könnten. Gerade am Studienanfang wissen Studierende oft nicht, nach welchen Regeln sich neue Texte aus vorhandenen wissenschaftlichen Erkenntnissen herstellen lassen (vgl. Kruse/Ruhmann 1999: 109). Vielen falle es deshalb schwer, so Furchner et al. (1999 $:$ 64), "sich durch Exzerpieren ein sicheres Textverständnis zu erarbeiten, die Textinformation unter dem Gesichtspunkt ihrer eigenen Fragestellungen zu reduzieren, verlässlich und eigenständig Bericht über die gelesenen Positionen zu erstatten und sie von den eigenen Gedanken zum Thema zu trennen".

Moll (2002) stellt eine differenzierte Liste von "Problembereichen" auf, wobei sie zunächst bereits von Steets genannte Schwierigkeiten nennt: Gliederungsprobleme, Textverstehen, Nachvollzug der Argumentation und Erkennen des Wesentlichen. Die Liste sei hier wiedergegeben, weil sie das Spektrum der Probleme weitgehend abdeckt:

- Argumentationsverlauf durch zu starke Verkürzung und Auslassungen nicht mehr nachvollziehbar;

- Argumentationsverlauf durch fehlende Verknüpfungen (logische Relationen) nicht mehr nachvollziehbar;

- das Exzerpt ist so umfangreich und eng an den Primärtext angelehnt, dass es seinen Zweck des "optimierten Wissenstransfers" nicht mehr erfüllt;

- starres Festhalten an der Gliederung des Primärtextes, was häufig einer sinnvollen Reduktion entgegensteht;

- beim Versuch, sich von Primärtext zu lösen und eigene Formulierungen zu finden, kann sich die "Informationsqualität" verändern;

- Schwierigkeiten bei der Verwendung von Indikativ/Konjunktiv (indirekte Redewiedergabe) bzw. Präsens/ Präteritum; 
- Schwierigkeiten bei der Verwendung der "alltäglichen Wissenschaftssprache";

- keine vollständige Literaturangabe auf dem Exzerpt;

- wörtliche Zitate sind optisch nicht von eigenen Formulierungen zu unterscheiden;

- fehlende Seitenangaben bei wörtlichen Zitaten;

- unübersichtliche Seitengestaltung und fehlende Gliederung durch Absätze.

Bei einer Umfrage an der ALU Freiburg berichteten 230 von 284 befragten Studierenden von Schreibproblemen (vgl. Dittmann et al. 2003). Von diesen wiederum kannten 43,8\% Probleme mit dem Zusammenfassen von Texten der Forschungsliteratur. Damit nimmt diese Problemkategorie auf einer Rangliste von 15 vorgegebenen Problemen den siebten Rang ein. Eine nahe liegende Vermutung wäre nun, die Probleme mit dem Exzerpieren auf den oft komplexen und deshalb schwer verständlichen Inhalt der Forschungsliteratur zurückzuführen. Allerdings stehen bei der genannten Umfrage Probleme mit dem Inhalt der Forschungsliteratur erst auf Rang 11; d. h., nur 35,7\% der Studierenden mit Schreibproblemen haben auch Schwierigkeiten mit dem Inhalt der Forschungsliteratur. Dies zeigt, dass solche Probleme zwar durchaus vorhanden sind, so dass die Schwierigkeiten, die manche Studierende mit dem Exzerpieren haben, (auch) hierauf zurückgeführt werden können. Das Gros der Studierenden scheint aber, so der Schluss von Dittmann et al. (2003: 181), "zumindest in der Selbsteinschätzung [...] mit den fachlichen Inhalten durchaus zurande [zu] kommen", so dass "entgegen einem landläufigen Vorurteil" die Probleme der Studierenden mit dem akademischen Schreiben "nicht primär im Zugang zum Fachwissen wurzeln". Empirisch abgesichert sind weiterhin Zusammenhänge zwischen einzelnen Aspekten des Schreibprozesses und dem Exzerpieren: Dittmann et al. (2003: 178) fanden bei ihrer Befragung signifikante Zusammenhänge

- zwischen Schwierigkeiten mit dem Exzerpieren von Forschungsliteratur und dem Problem, den Einstieg in das Schreiben einer Hausarbeit zu finden, und

- zwischen Schwierigkeiten mit dem Exzerpieren und dem Problem, den Überblick über den Inhalt der entstehenden Arbeit zu behalten.

Denkt man darüber nach, wie die Exzerpierfähigkeit der Studierenden verbessert werden kann, so stellt sich zunächst die Frage, ob der Stellenwert dieser Fähigkeit an den Hochschulen überhaupt erkannt wird. Ehlich (1981: 379) kam noch zu dem Resultat, Texte über Texte seien in der allgemeinen Wertschätzung von vornherein belastet, was bedeute, dass die Textgruppe, zu der das Exzerpt gehört, in der wissenschaftlichen Analyse kaum Beachtung finde. Die Einschätzung dieser Textgruppe muss sich in den folgenden 20 Jahren geändert haben, denn eine 1999 an der LMU München durchgeführte Umfrage förderte zutage, dass immerhin $60 \%$ der befragten HochschullehrerInnen die Fähigkeit zu exzerpieren als relevant für den Studienerfolg einstuften; nur 10\% erachteten ihn als weniger wichtig (vgl. Ehlich/Steets 2000 ${ }^{\mathrm{a}}$. Mit den praktischen Konsequenzen für die Didaktik sieht es allerdings anders aus: Danach befragt, ob das Exzerpieren im Grundstudium vermittelt werde, gaben nur $11 \%$ der HochschullehrerInnen eine positive Antwort. Damit einher geht die Tatsache, dass nur äußerst selten Übungsexzerpte eingefordert werden, nämlich nur von $12 \%$ der Lehrenden im Grundstudium und von 11\% im Hauptstudium. Entsprechend schlecht informiert sind die Lehrenden denn auch über die Exzerpierfähigkeit der Studierenden: 57\% der Befragten gaben an, diese nicht einschätzen zu können, $5 \%$ halten sie für gut, $27 \%$ für hinreichend und $11 \%$ für schlecht. Die Diskrepanz zwischen der Einschätzung der Bedeutung des Exzerpierens und der Lehrpraxis ist also groß: Selbst bei den Naturwissenschaftlern, den Mathematikern und den Juristen der LMU, bei denen die Technik des Exzerpierens überhaupt nicht vermittelt wird, sind immerhin $45 \%$ bzw. $40 \%$ bzw. 25\% der Ansicht, dass Exzerpieren-Können den Studienerfolg maßgeblich mitbestimmt. 
Wie wird den Studierenden geholfen? Zum einen gibt es Schreibberatungen an deutschen Hochschulen, deren Angebote sich an Studierende mit Schreibproblemen richten (vgl. den Sammelband Kruse et al. 1999). Das Exzerpieren-Lernen sollte freilich als Normalfall gelten, darf also nicht nur im Kontext von Schreibproblemen thematisiert werden. Überhaupt führt die gängige Praxis dazu, dass Schreibprobleme eher als individuelles Versagen und nicht "als individueller Ausdruck eines allgemeinen Missstandes" empfunden werden (Kruse 1998: 29), was meist passender wäre. Weiterhin gibt es eine breite Ratgeberliteratur. Hier ist es für die Studierenden nicht einfach, Brauchbares von weniger Brauchbarem $\mathrm{zu}$ unterscheiden: Probleme der Ratgeberliteratur diskutiert u. a. Jakobs (1999: 179-182). ${ }^{5}$ Auch das Angebot im Internet ist durchwachsen. So findet man auf einer Seite der Universität Salzburg - also mit einer gewissen Autorisierung - seit einigen Jahren den von Klaus Zelewitz verfassten, auf einer falschen Auffassung vom Exzerpieren basierenden und irreführenden Hinweis, dieses sei anachronistisch geworden 6 :

Im Zeitalter von Fotokopie und Scannen ist auch beim Exzerpieren ein Wandel eingetreten: Das tageoder wochenlange fein-säuberliche Abschreiben von seitenlangen Texten in Kanzlistenart ist anachronistisch geworden. Das eigene Exemplar ist in vielen Fällen erschwinglich, die Photokopie ist manuellen [sic] oder maschinellen [sic] Abschreiben sowohl aus arbeitsökonomischen Gründen als auch aus solchen der geringeren Fehleranfälligkeit weit überlegen. Zusätzlich zeigen nur Original (fotomechanische Reproduktion, Scan) auch das Layout, insbesondere Tabellen und Abbildungen!

Nach Einschätzung von Moll, wohl auf der Grundlage informeller Befragungen an der LMU, korrespondiert hiermit eine negative Bewertung des Exzerpierens durch Studierende: Im Zeitalter schneller elektronischer Reproduktionsmöglichkeiten sei das Exzerpieren aus studentischer Sicht "zu einer unpopulären Form der Wissensaneignung geworden, die als 'altmodisch' und 'ineffizient' in Misskredit geraten" sei. Erkennbar sei dies nicht zuletzt daran, dass den Studierenden der Begriff "Exzerpt" häufig unbekannt sei (Moll 2002: 104).

In der Regel setzt, so Furchner et al. $\left(1999^{\mathrm{b}}\right.$ : 62) die Universität voraus, "dass die Studierenden durch ihre Schulausbildung hinreichend auf das Schreiben wissenschaftlicher Texte vorbereitet seien" - und dies, "obwohl das Verfassen von Studienarbeiten Fertigkeiten verlangt, die im schulischen Kontext nicht relevant sind und dementsprechend dort nicht angeeignet und eingeübt werden". Ludwig (2003: 240) meint darüber hinaus, im Aufsatzunterricht an Deutschlands Schulen sei "der Bezug zum akademischen Schreiben [...] völlig aus dem Blick geraten", und die Untersuchung der Vorgaben durch die "Einheitlichen Prüfungsanforderungen im Fach Deutsch" durch Beste (2003) ergab das Fazit, die typischen Schreibaufgaben in der Oberstufe seien nur mit Einschränkung als Vorbereitung auf die Hochschule zu betrachten (vgl. Beste 2003: 280). Ihre prinzipielle Grenze hat die Vermittlung der Fähigkeit akademischen Schreibens in der Schule ohnehin dort, wo die wissenschaftlichen Stile der Einzeldisziplinen ins Spiel kommen - so gesehen ist das akademische Schreiben eine Tätigkeit sui generis und muss dementsprechend auch an der Universität vermittelt werden (vgl. Dittmann et al. 2003: 169f.). Zwar sollten die angehenden Studierenden von der Schule schriftsprachliche Basiskompetenzen mitbringen - es sei dahingestellt, ob dieser Anspruch eingelöst wird; doch bleibt, wie Steets (1999: 399-402) zeigt, in den gymnasialen DeutschLehrplänen unklar, "welche Rolle dem Schreiben in wissenschaftspropädeutischer Hinsicht zugeordnet ist". Das Exzerpieren betreffend stellt sie fest, dass in den Lehrplänen und Unterrichtswerken dessen Beherrschung in der Kollegstufe schlicht vorausgesetzt werde. Außerdem komme eine Geringschätzung dieser als "Arbeitstechnik" klassifizierten Fähigkeit zum Ausdruck, wie sie auch in der wissenschaftlichen Literatur zu finden sei (ebd.: 406f.).

\footnotetext{
5 Gute Tipps zum Exzerpieren finden sich bei Stary/Kretschmer (1994: 114-120.), Bünting et al. (1996: 32-34.) und Esselborn-Krumbiegel (2006: 84-86.). Für die Sekundarstufe vgl. Becker-Mrotzek/Böttcher (2006). Die Exzerpt-Definition von Karmasin/Ribing (2006: 84) hingegen ist höchst problematisch.

${ }^{6}$ http://www.sbg.ac.at/ger/zelewitz/lv/ws0102/exzerpt.htm, Stand 02.01.2008.
} 
Dem hält Steets entgegen, dass die "Tätigkeit des Exzerpierens [...] grundlegende Kompetenzen [schult], die man gern als Schlüsselqualifikationen bezeichnet, die überall dort erforderlich sind, 'wo es um intellektuell anspruchsvolle Tätigkeiten, um selbstständiges Denken, um eigenständiges Tun im Studium und im Beruf geht'" (ebd.: 408). Unter didaktischem Gesichtspunkt schlägt sie eine Verbindung zwischen dem Erwerb der Exzerpierfähigkeit mit der Behandlung von Argumentationsverfahren vor - aus unserer Sicht ist das eine sehr sinnvolle Kopplung.

Inwieweit Studierende die Technik des Exzerpierens als Teilfähigkeit des akademischen Schreibens bereits von der Schule mitbringen, ist die leitende Fragestellung der im Folgenden referierten empirischen Studie.

\section{Die empirische Studie}

\subsection{Fragestellungen, Textauswahl und Durchführung der Studie}

Die folgende Studie verfolgt den Zweck herauszufinden, welche Exzerpier-Kenntnisse SchülerInnen während ihrer Schulzeit vermittelt bekommen, welche Kenntnisse sie also an die Universität mitbringen. Die Ergebnisse sind deshalb sowohl für die Schule als auch für die Universität interessant: Für die Schule z. B. in Bezug auf die Frage, ob die hinsichtlich der Vermittlung von Textreproduktionskenntnissen gesteckten Ziele erreicht wurden, für die Universität - und hierauf liegt unser Hauptaugenmerk - im Hinblick auf die Frage, welche Kenntnisse bei den Studierenden vorausgesetzt werden können. Als Arbeitshypothese setzen wir voraus, dass Studierende in der Regel Probleme mit dem Exzerpieren haben. Auf der Grundlage dieser Hypothese lautet die mit der Studie verfolgte Fragestellung:

Wie gut können SchülerInnen der Jahrgangsstufe 13 exzerpieren? D. h.: Inwieweit sind sie in der Lage, die Argumentationsstrukturen eines anspruchsvollen expositorischen Textes nachzuvollziehen, die wichtigsten Aussagen zu rekonstruieren und diese sinnvoll und strukturiert wiederzugeben?

Der empirische Ansatz dieser Studie ist ein zweifacher, nämlich erstens eine textlinguistisch fundierte Analyse von Exzerpten (Abschnitte 4.2-4.5) und zweitens eine Fragebogenstudie zur subjektiven Einschätzung der konkreten Exzerptaufgabe sowie zum Exzerpieren allgemein (Abschnitt 4.6). In der textlinguistischen Analyse wurden von SchülerInnen verfasste Exzerpte auf ihre Qualität hin analysiert und es wurde erfasst, welche Arten von Fehlern in welchem Ausmaß auftraten. Zugleich wollen wir mit diesem Vorgehen zeigen, dass die Textlinguistik Mittel bereitstellt, die Qualität von Textexemplaren intersubjektiv reliabel zu bestimmen: Jeder Analyseschritt ist präzise definiert und kann somit zur Überprüfung der Interraterreliabilität auch von mehreren AuswerterInnen vollzogen werden. ${ }^{7}$

Als Primärtext musste ein expositorischer Text ausgewählt werden, dessen Inhalt nicht zu spezifisch sein durfte - ein wissenschaftlicher Fachtext, wie man ihn Studierenden hätte vorlegen können, kam deshalb nicht in Frage - sondern der mit einem durchschnittlichen Weltwissen, wie es 18- bis 19-jährige SchülerInnen in der 13. Klasse eines allgemeinbildenden Gymnasiums besitzen, zu verstehen war. Diese Voraussetzung erfüllt der ausgewählte Text aus der "Süddeutschen Zeitung" vom 15.01.2002, Rubrik "Themen aus Deutschland", mit dem Titel "Die Debatte über die Zulässigkeit der Forschung an Embryonen. Ein zelluläres Gebilde wie du und ich" von Heidrun Graupner. ${ }^{8}$ Die SchülerInnen hatten vorgängig im Biologieunterricht, z. B. in den Lehrplaneinheiten "Aspekte der Molekulargenetik" und

\footnotetext{
${ }^{7}$ In dieser Studie haben wir auf eine strenge Überprüfung der Interraterreliabilität verzichtet und uns mit der Diskussion jedes einzelnen Analyseschrittes begnügt.

${ }^{8}$ Der Text ist in Anhang 1 abgedruckt.
} 
"Angewandte Genetik", so viel Wissen zum Thema erlangen können, dass das Verständnis des Textes gewährleistet sein sollte. Unterstellt, dass das Textverständnis für alle ProbandInnen in gleicher Weise gegeben war, sollte die Aufgabenstellung es ermöglichen, die reine Exzerpierfähigkeit zu messen. Eine solche Voraussetzung ist erkennbar eine Idealisierung: Tatsächlich ist der Kenntnisstand auch in derselben Klasse/im selben Kurs unterschiedlich. Deshalb haben wir die Vorkenntnisse erhoben und das Ergebnis in die Auswertung einbezogen (vgl. Abschnitt 4.6.2, zu Fragebogen 2, Frage 11). Die Aufgabenstellung war definiert als Anfertigung eines Exzerpts als Grundlage eines Referates für den Ethik- und Gemeinschaftskundeunterricht.

Für die Erfassung der subjektiven Einschätzung durch die ProbandInnen - dem zweiten empirischen Ansatz dieser Studie - wurden zwei Fragebögen entwickelt. Sie sollten Aufschluss darüber geben, welche externen und internen Faktoren zum Gelingen bzw. zum Scheitern der Exzerpier-Aufgabe beigetragen hatten. Hierzu wurden den SchülerInnen sowohl geschlossene, quantitativ auszuwertende Fragen (z. B. die Frage nach vorhandenen Kenntnissen) als auch offene, qualitativ auszuwertende Fragen (z. B. die Aufforderung zur Erläuterung von Problemen bei der Erstellung des Exzerpts) gestellt. Außerdem sollte mit den Fragen herausgefunden werden, welche Aspekte des Primärtextes einerseits (z. B. Argumentationsstrukturen, Sprache etc.) und des Exzerpierens selbst andererseits (z. B. Herausarbeiten der einzelnen Argumente, Kürzen des Textes etc.) den SchülerInnen Probleme bereiteten (Fragebogen 2).

Neben diesen Fragen zum konkreten Exzerpt wurde ein weiterer Fragebogen mit allgemeinen Fragen zum Exzerpieren entwickelt, der unter anderem Aufschluss darüber geben sollte, inwieweit sich die SchülerInnen der Wichtigkeit des Exzerpierens bewusst waren bzw. inwiefern sie es in ihrem Schulalltag nutzten. Auch war es wichtig zu erfahren, wie viele und welche SchülerInnen nach dem Abitur ein Studium beginnen wollten, da die Studie ja vor allem zeigen möchte, welche Exzerpierkenntnisse StudienanfängerInnen von der Schule mitbringen (Fragebogen 1). ${ }^{9}$ Es erübrigt sich, hier auf die Fragen im Einzelnen einzugehen, da diese bei der Auswertung besprochen werden (vgl. Abschnitt 4.6).

Für die Durchführung der Studie wurden an einem süddeutschen allgemeinbildenden Gymnasium ein Deutsch-Grundkurs (GK) mit 21 SchülerInnen und ein Deutsch-Leistungskurs (LK) mit 12 SchülerInnen ausgewählt.

Die Zusammensetzung der Gruppe nach Alter und Geschlecht gibt die folgende Tabelle wieder, und zwar in absoluten Zahlen und prozentual ${ }^{10}$

\begin{tabular}{|c|c|c||c|c|}
\hline & 18 Jahre & 19 Jahre & Männlich & Weiblich \\
\hline $\begin{array}{c}\text { Anzahl der } \\
\text { SchülerInnen (abs.) }\end{array}$ & $13(3)$ & $20(9)$ & $15(4)$ & $18(8)$ \\
\hline Anteil (in \%) & $39,4(25)$ & $60,6(75)$ & $45,5(33,3)$ & $54,6(66,7)$ \\
\hline
\end{tabular}

Tabelle 1: Die ProbandInnen (in Klammern: LK)

Fragebogen 1 wurde einige Tage vor dem eigentlichen Test ausgefüllt, nachdem die LehrerInnen der Kurse sicher gestellt hatten, dass alle ProbandInnen wussten, was man unter "Exzerpt" versteht. Für den eigentlichen Test, den G. F. selbst leitete, standen in jedem Kurs 105 Minuten während der regulären Unterrichtszeit zur Verfügung. Nach der Lektüre des Primärtextes und der Aufgabenstellung konnten die SchülerInnen Fragen stellen. Die im Primärtext enthaltenen Fremdwörter Zäsur und Nidation wurden in beiden Kursen erklärt. Zum Erstellen des Exzerptes hatten die SchülerInnen ca. 75 Minuten Zeit, in den verblei-

\footnotetext{
${ }^{9}$ Die Fragebögen sind in Anhang 2 und Anhang 3 abgedruckt.

${ }^{10}$ Summen über 100 gehen auf Rundungsdifferenzen zurück.
} 
benden ca. 15 Minuten füllten sie Fragebogen 2 aus. Fragebögen und Exzerpte wurden anonymisiert, doch bekam jede/r SchülerIn eine zweistellige Zahl zugeordnet, so dass Exzerpt und Fragebogen aufeinander bezogen werden konnten.

\subsection{Die textlinguistische Analysemethode}

Die Auswertung der Exzerpte orientiert sich zunächst an der Propositions-Theorie nach Kintsch und van Dijk und den von diesen Autoren vorgeschlagenen Makroregeln (vgl. Abschnitt 2.2). Weitere Analyseparadigmen sind der "Rhetorical Structure Theory" (vgl. Mann/Thompson 1988; vgl. dazu Abschnitt 4.3.3) und dem "Zürcher Textanalyseraster" (vgl. Nussbaumer/Sieber 1994; vgl. dazu Abschnitt 4.3.3) entnommen. Das Ansetzen bei der Propositions-Theorie lässt sich erstens mit der Relevanz der Proposition als Verarbeitungseinheit in der Sprachrezeption rechtfertigen (vgl. u. a. Rickheit/Strohner 1993: 231-233.), zweitens und vor allem aber damit, dass die Makroregeln nach van Dijk (1980: 44) als eine "Rekonstruktion des Teils unseres Sprachvermögens" zu verstehen sind, "mit dessen Hilfe wir Bedeutungen zu größeren Bedeutungsganzheiten zusammenfügen" - wie wir gesehen haben, eine entscheidende Teiltätigkeit beim Exzerpieren. Auch Keseling (1993: 5f.) stellt in seiner Arbeit über Zusammenfassungen fest, dass das Verstehen eines Textes mehr oder weniger automatisch mit der Bildung von Makropropositionen einhergehe, und er folgert, dass auch die Schreiber von Zusammenfassungen solche Operationen - nämlich die Makroregeln anwenden, "und zwar [...] nicht erst beim Schreiben, sondern bereits beim (ersten) Lesen des Primärtextes" (ebd.: 6). Für unsere Zwecke können wir die Makroregeln demnach als eine Rekonstruktion der gedanklichen Leistung ansehen, die die SchülerInnen beim Exzerpieren vollbringen müssen.

Um die Schülerexzerpte nicht nur analysieren, sondern auch bewerten zu können, unterzogen wir den Zeitungstext zunächst einer Musteranalyse mittels der Propositions-Theorie. Dabei wurde die Makropropositions-Analyse auf drei hierarchische Ebenen beschränkt. Die Bildung noch höherer, 'globalerer' Makropropositionen führt zu einer Verknappung der Information des Textes, die kein nachvollziehbares Exzerpt mehr liefert. Konkret wurden folgende Makroregeln im Sinne van Dijks (1980: 45-56.) zur Analyse verwendet: ${ }^{11}$

\section{Regel I: AUSLASSEN}

Jede in Bezug auf die Interpretation des Textes auf einer jeweils globaleren Ebene irrelevante, nicht-essenzielle Information kann ausgelassen werden. Bei gegebener Propositionenreihe $\langle\alpha, \beta, \gamma\rangle$ dürfen $\alpha$ und $\gamma$ gestrichen werden, wenn sie für den Text - in einer globaleren Bedeutung als der der einzelnen Propositionen - keine 'Funktion' mehr haben, beispielsweise als Präsuppositionen für die Interpretation folgender Propositionen. Schematisch also: $\langle\alpha, \beta, \gamma\rangle \rightarrow \beta$. Die Propositionenreihe (i) EIN MäDCHEN LIEF VORBEI. (ii) SIE TRUG EIN KLEID. (iii) DAS KLEID WAR GELB. kann nach Regel I zu (iv) EIN MÄDCHEN LIEF VORBEI. reduziert werden, wenn es für den Text im Weiteren keine Rolle mehr spielt, dass das Mädchen ein Kleid und keine Jeans trug und, a fortiori, dass das Kleid gelb war. Dieses Beispiel geht auf van Dijk (1980: 46) zurück. Propositionen kennzeichnen wir im Folgenden in der Regel durch Kapitälchen. Für die Interpretation des Textes ist insoweit diese Information irrelevant. Außerdem sind die ausgelassenen Informationen insofern nicht-essenziell sondern quasi "zufällig", als "trägt ein Kleid" keine wesentlicher Teil des Begriffs 'Mädchen' und "ist gelb" kein wesentlicher Teil des Begriffs "Kleid" ist. Nach der Anwendung von Regel I ist ein Teil

11 Auch van Dijk (1980: 56-67) setzt diese vier Regeln zur Analyse eines Zeitungsartikeltextes ein. In anderen Untersuchungen nennt er weitere Regeln, doch ist das für unseren Zusammenhang ohne Belang. 
der Information der Mikroproposition unwiederbringlich verloren, kann also nicht zurückerschlossen werden.

\section{Regel II: SELEKTIEREN}

Das ist bei der Regel SELEKTIEREN anders: Zwar wird auch hier eine gewisse Informationsmenge ausgelassen, aber sie kann aufgrund allgemeiner Kenntnisse der Situation, der Handlung oder des Geschehens bzw. aufgrund semantischer Postulate für Konzepte rekonstruiert werden. Schematisch sieht das aus wie bei Regel I: $\langle\alpha, \beta, \gamma\rangle \rightarrow \beta$, nur dass jetzt $\alpha$ und $\gamma$ nur gestrichen werden dürfen, wenn $\beta$ die Reihe $\langle\alpha, \gamma\rangle$ impliziert, $\alpha$ und $\gamma$ also Bedingungen, Bestandteile, Präsuppositionen oder Folgen der nicht ausgelassenen Proposition $\beta$ sind. Mit einem Beispiel von van Dijk (1980: 47): Die Propositionenreihe (i) PETER LIEF ZU SEINEM AUTO. (ii) ER STIEG EIN. (iii) ER FUHR NACH FRANKFURT. kann durch SELEKTIEREN auf (iii) reduziert werden, da (i) und (ii) Bedingungen bzw. Bestandteile von (iii) enthalten.

\section{Regel III: GENERALISIEREN}

Hier werden mehrere Propositionen durch eine neue ersetzt, wobei, wie in der Anwendung von Regel I, Information verloren geht. Schematisch: $\langle\alpha, \beta, \gamma\rangle \rightarrow$ @. Im Unterschied zu Regel I werden hier aber essenzielle, "merkmalskonstituierende" Komponenten eines Konzepts ausgelassen, weshalb van Dijk (1980: 48) diesen Vorgang auch als "Abstraktion" bezeichnet. Das ist paradigmatisch dann der Fall, wenn sich Begriffe mehrerer Propositionen unter einen Oberbegriff subsummieren lassen. Mit van Dijks Beispiel: (i) EINE PUPPE LAG AM Boden. (ii) EIne Holzeisenbahn lag am Boden. (iii) BAusteine lagen AM Boden. Reduziert zu: (iv) SPIElzEug LAG AM BodEN.

\section{Regel IV: KONSTRUIEREN oder INTEGRIEREN}

Diese Regel ähnelt nach van Dijk $(1980,48)$ in der Funktion Regel II, operiert aber wie Regel III nach dem Schema $\langle\alpha, \beta, \gamma\rangle \rightarrow \mathrm{Q}$ : Information wird durch neue Information ersetzt, nicht ausgelassen oder selegiert. Zwischen den Begriffen der Propositionenreihe besteht ein inhärenter Zusammenhang, nämlich "gewöhnliche Bedingungen, Umstände, Komponenten, Folgen u.ä. eines Zustands, Geschehens, Prozesses, einer Handlung u.ä.". Mit van Dijks Beispiel: (i) ICH GING ZUM BAHNHOF. (ii) ICH KAUFTE EINE FAHRKARTE. (iv) ICH STIEG IN DEN Zug EIN. (v) DeR Zug FUHR AB. wird reduziert zu: (v) ICH NAHM DEN Zug. Die Propositionen (i) - (iv) enthalten obligatorische und optionale Elemente unseres "konventionellen Wissens, des Rahmens ["frames"] ZugREISE. Der Begriff "Zugreise" muss also im Text gar nicht vorkommen, wir können ihn aus den im Text vorgefundenen Bestandteilen ableiten.

Regel IV ist von großer Relevanz: Durch sie wird die Integration von Wissensrepräsentationskonzepten in die Propositionstheorie möglich, die deren Beschränkungen überwinden können. Diese Beschränkungen einer reinen Propositionstheorie des Textverstehens können wir hier nicht diskutieren, verweisen aber auf die Darstellung bei Schnotz (1988), der eine Erweiterung mittels des Konzepts der "mentalen Modelle" im Sinne von Johnson-Laird (1983) vorschlägt: Die/der TextrezipientIn bildet "neben der propositionalen Repräsentation [...] auch ein sog. Situationsmodell [...] Dabei handelt es sich um ein mentales Modell des im Text beschriebenen Sachverhalts, das durch Integration der Textinformation mit dem bereits vorhandenen Sachwissen des Lesers gebildet wird" (Schnotz 1988: 306).

Auf die Bildung der Makropropositionen wurden zwei weitere Kriterien angewendet. Erstens wurde die Textsorte des Primärtextes berücksichtigt: Bei einem Zeitungsartikel, der über ein aktuelles, kontroverses gesellschaftspolitisches Thema mit wissenschaftlichem Hintergrund berichtet, spielen auch für eine Zusammenfassung die Aktualität der wiedergegebenen Sachverhalte und die sich daraus ergebenden kontroversen Standpunkte eine zentrale Rolle, 
der chronologische Verlauf der beschriebenen Debatte hingegen ist zweitrangig. Zweitens wurde die Relevanz der wiedergegebenen Informationen im Hinblick auf die schulische Aufgabenstellung - ein Exzerpt für ein Referat - bewertet. Dazu gehört z. B., dass für eine an das Referat anschließende Diskussion des Themas im Ethik- oder Gemeinschaftskundeunterricht die jeweiligen Argumente und Gegenargumente klar herausgestellt werden.

Die Zusammenfassung des Textes auf drei Ebenen führte zu 47 Makropropositionen auf der ersten, 25 auf der zweiten und 9 auf der dritten Ebene (siehe Anhang 4). Auf der Grundlage dieser Analyse wurde ein "ideales Exzerpt" erstellt, das sich vorwiegend auf Makropropositionen der 2. Ebene stützt, und es wurde festgelegt, dass die Schülerexzerpte nicht detaillierter als dieses "ideale Exzerpt" sein sollten ${ }^{12}$ (siehe Anhang 5).

\subsection{Vorgehensweise bei der Auswertung}

Die Auswertung der Schülerexzerpte erfolgte nach drei Hauptbewertungskriterien: Ein Exzerpt durfte nicht zu detailliert sein, es musste dennoch alle wichtigen Informationen des Primärtextes enthalten, und diese mussten sinngemäß richtig wiedergeben werden.

\subsubsection{Ermittlung fehlender Makropropositionen}

Die Frage, welche Informationen - Makropropositionen - im Schulerexzerpt fehlen, kann nur vor dem Hintergrund der Detailliertheit des Exzerpts beantwortet werden: je detaillierter ein Exzerpt ist, desto mehr Makropropositionen der zweiten, nicht aber der dritten Ebene muss es beinhalten. Dabei kommt es selbstverständlich nicht auf die wörtliche Übereinstimmung der im Schülerexzerpt enthaltenen Makropropositionen mit denen unserer Propositionsanalyse an: die Makropropositionen der Tabelle in Anhang 4 können theoretisch auf unbegrenzt viele Arten in konkreten Text umgesetzt werden. So lautet M2;16 aus der Tabelle: "Gegner der ESForschung sind der Ansicht, dass der Embryo von Anfang an das Recht auf Leben habe." Der folgende Satz aus einem Schülerexzerpt wurde als M2;16 - diese mithin als "vorhanden" gewertet (Exzerpt 34): "Die Gegner sind allerdings der Meinung, dass ein Embryo von Anbeginn [seiner Entwicklung] 'Träger der Menschwürde' sei."

Des Weiteren ist es möglich, Propositionen auf andere Weise zu Makropropositionen zusammenfassen, als in unserer Analyse geschehen. Für jede im Schülerexzerpt enthaltene Information, die nicht direkt einer Makroproposition aus der Tabelle (Anhang 4) entsprach, wurde deshalb geprüft, ob sie - z. B. aufgrund der Anwendung einer anderen Makroregel durch eine mögliche alternative Zusammenfassung von Propositionen zustande gekommen sein konnte. In der Auswertung wurde diese Information dann der Makroproposition aus der Tabelle zugeordnet, der sie am ehesten entsprach.

Schließlich wurde berücksichtigt, dass durch Kontext und Reihenfolge der Präsentation von Informationen in den Schülerexzerpten Makropropositionen impliziert sein können. So formuliert z. B. der Schüler in Exzerpt Nr. 24: "Nur einen theoretischen Lösungsansatz konnte ein Neurophysiologe schon nennen: es müsste auch möglich sein, von einem wenige Tage alten Embryo Stammzellen zu gewinnen, ohne ihn zu töten." Da diese theoretische Möglichkeit am Schluss genannt wird und durch die Verwendung von nur, ist impliziert, dass dies die (bisher einzige) Lösung des "ethischen Dilemmas von Forschung, Politik und Justiz" ist - so das Zitat aus dem Primärtext in M2;5 unserer Tabelle. Diese Makroproposition wird also als "vorhanden" gewertet.

Die Ermittlung fehlender Makropropositionen wurde vor dem Hintergrund der theoretischen Möglichkeiten vorgenommen, ein "vollständiges" Exzerpt zu formulieren. Diese Möglichkeiten sind:

\footnotetext{
12 Auf Ausnahmen von dieser Forderung gehen wir in Abschnitt 4.3.2 ein.
} 
1. Der Exzerpierende verwendet alle Makrooppositionen der zweiten Ebene aus der Tabelle detailliertes Exzerpt.

2. Die Exzerpierende verwendet alle Makropropositionen der ersten Ebene der Tabelle - zu detailliertes Exzerpt.

3. Der Exzerpierende verwendet alle Makropropositionen der dritten Ebene der Tabelle sehr knappes Exzerpt.

4. Die Exzerpierende verwendet Makropropositionen aller (oder von zwei der drei) Ebenen der Tabelle, aber so, dass folgende Kriterien berücksichtigt werden: (i) Jede Makroproposition der dritten Ebene musste in irgendeiner Form vorhanden sein, wobei "in irgendeiner Form" bedeutet, dass sie auch durch entsprechende Propositionen einer niedrigeren Ebene ausgedrückt werden konnte. (ii) Bestimmte Makropropositionen konnten weggelassen werden, wenn die Auslassung systematisch herleitbar war. Für eine Pro- und Kontraargumentation z. B. bedeutet dies, dass ein Pro-Argument mit etwa demselben Grad an Detailliertheit (wie Hintergrundinformation oder Begründungen) dargestellt werden musste wie ein Kontraargument. Entsprechende Informationen mussten also symmetrisch vorhanden sein oder fehlen. (iii) Der Grad der Detailliertheit musste ungefähr beibehalten werden: Wenn beispielsweise in der ersten Hälfte des Exzerpts alle Makropropositionen der zweiten Ebene genannt wurden, durfte gegen Ende z. B. die Einheit M2;2513 nicht ausgelassen werden.

\subsubsection{Ermittlung von "Detailliertheitsfehlern"}

Ein Exzerpt kann in dreierlei Hinsicht zu detailliert sein:

1. Es kann Aussagen enthalten, die keiner Aussage des Primärtextes entsprechen, die aber auch nicht eine - sinnvolle - Funktion der Textorganisation übernehmen (z. B. als Kommentar). In den untersuchten Schülerexzerpten kamen z. B. Kommentar vor, die mit dem ursprünglichen Text nichts zu tun hatten; diese zählten als Fehler. So lautet etwa der erste Satz von E 35: "Der Afghanistan-Krieg ist in den Hintergrund getreten." Man darf vermuten, dass dieser Satz, der keinerlei Bezug zum Thema hat - eben weil er keinen Bezug zum Thema hat - als Provokation intendiert war.

2. Es kann Aussagen zu wenig oder auch gar nicht zusammengefasst wiedergeben, indem es

- (partiell) aus Makropropositionen der 1. Ebene zusammengesetzt ist,

- Aussagen direkt von der Textebene übernimmt.

Nicht in allen Fällen galt allerdings die Übernahme von Makropropositionen der 1. Ebene in das Schülerexzerpt als Fehler, auch wenn sie eine Abweichung vom idealen Exzerpt darstellten. Es sollte den SchülerInnen nämlich gestattet sein, gewisse Akzentsetzungen, z. B. entsprechend einem bestimmten Interesse am Thema, vorzunehmen. So konnten z. B. die in M1;19 genannten drei Zeitpunkte, ab denen der Embryo als Mensch gilt, als Ergänzung zu M2;9 ins Exzerpt aufgenommen werden, denn in der Betonung der biologischen Dimension der Thematik drückt sich ein legitimer Interessenbereich aus.

3. Es kann Wiederholungen bzw. sinngemäß identische Aussagen enthalten. So wurde z. B. in E 23 vor der Darstellung der unterschiedlichen Meinungen zur Frage, wann ein Embryo ein Mensch sein, gesagt, dass es "mehrere unterschiedliche Antworten" gebe - eine Aussage, die das Folgende vorstrukturiert und somit nicht als zu detailliert gewertet wurde. Etwas weiter unten im Text heißt es dann: "Die Antworten sind umstritten." Diese Aussage wurde als Wiederholung und damit als Detailliertheitsfehler gewertet, allerdings nur als halber Fehler,

13 M2;25: "Vergleiche mit Verhütung oder Abtreibung (wie sie oft gemacht wurden) sind in diesem Zusammenhang nicht sinnvoll." 
da die Vergabe eines ganzen Fehlers für die vollständige Wiederholung eines längeren Textsatzes reserviert war.

\subsubsection{Ermittlung von Sinnfehlern}

Methodologisch betrachtet ist die Ermittlung von Sinnfehlern heikel, in der Auswertungspraxis, also bei gegebenem Primärtext, ist gleichwohl eine reliable Bestimmung möglich. In der vorliegenden Studie wurde zwischen schweren (oder "ganzen") und leichteren (oder "halben") Sinnfehlern unterschieden. Ein ganzer Fehler wurde berechnet, wenn eine Aussage die Information des Primärtextes so sehr entstellte, dass dies entweder das Verständnis des weiteren Exzerpttextes stark erschwerte oder die LeserInnen des Exzerpts keine Rückschlüsse auf die Bedeutung der entsprechenden Aussage im Primärtext hätten ziehen können. Als halber Sinnfehler wurde gewertet, wenn eine Information des Primärtextes zwar leicht sinnentstellt wiedergegeben worden war, dadurch aber das Verständnis des Exzerpts als Ganzes nicht beeinträchtig war bzw. die Leserin die globalere Bedeutung einer Aussage trotzdem noch hätte erschließen können.

An drei Typen von Sinnfehlern wollen wir das Vorgehen erläutern:

1. Die Beziehungen der unterschiedlichen 'Textspannen' ("text span" im Sinne von Mann/ Thompson 1988: 245) im Primärtext zueinander - bzw. der daraus ermittelten Makropropositionen (also u. a. der Argumente) zueinander - mussten im Exzerpt entsprechend wiedergegeben werden. Ob die jeweiligen Relationen in den Exzerpten mit denen des Primärtextes übereinstimmten, wurde analysiert, indem sämtliche Relationen von Primärtextspannen zueinander mit Hilfe der "Rhetorical Structure Theory" von Mann/Thompson (1988) ermittelt wurden und anschließend diese Relationen mit den in den Schülerexzerpten gefundenen verglichen wurden. ${ }^{14}$ So musste beispielsweise die Relation erkannt werden, durch die die Primärtext-Zeilen 1-12 mit den Zeilen 13-20 verbunden sind: Die Zeilen 13-20 stellen eine Art Hintergrundinformation für die Zeilen 1-12 dar, liefern also den Hintergrund, vor dem (bzw. auf Grund dessen) das, was in den Zeilen 1-12 beschrieben wird, stattfindet. Wenn die in Z. 1-12 beschriebenen anfänglichen Reaktionen aber beispielsweise falsch eingeordnet wurden, galt dies als Sinnfehler.

So heißt es am Beginn von E 35:

(8) "[...] kann man in den Medien eine Diskussion von ungeheurer Brisanz mitverfolgen. Forscher sprechen seit den 40. Bitburger Gesprächen gar von einem 'casus cnaxus', von einem 'entschlüsselten Menschen'."

Hier bezieht sich der Ausdruck "casus cnaxus", der im Originaltext nur auf die anschließend dargestellte neue "Möglichkeit" (Z. 13-16) gemünzt ist, auf die gesamte Debatte, auf die "Diskussion von ungeheurer Brisanz" - und jedenfalls nicht ausdrücklich auf die von J. Hescheler vorgestellte "Möglichkeit". Insofern ist die Relation, nämlich dass diese "Möglichkeit" den Hintergrund für die beschriebenen Reaktionen bildet bzw. auch für Teile davon, wie hier die Reaktion Roman Herzogs, nicht übernommen worden. Das allein wäre nur als halber Sinnfehler gewertet worden, weil der Satz die Information des Primärtextes nicht völlig sinnentstellt wiedergibt. Auch im Primärtext heißt es ja, bei dieser Debatte sei von einem "casus cnaxus" (Z.7) gesprochen worden, allerdings eben in einem deutlicheren Zusammenhang mit einem Ereignis als dies im Exzerpt dargestellt wird. Dieser Sinnfehler ist im vorliegenden Exzerpt aber mit einem weiteren verbunden: dass nämlich die Forscher "seit den 40. Bitburger Gesprächen [...] von einem 'entschlüsselten Menschen'" sprächen - was nicht stimmt; es war ja das "Thema" (Z. 5) der Gespräche, welches so formuliert war. Mithin

14 Dies im Einzelnen darzustellen würde den Rahmen des vorliegenden Artikels sprengen, doch wird das Vorgehen im Folgenden beispielhaft verdeutlicht. 
wurde für Satz (8) ein ganzer Sinnfehler berechnet, der sich, genau betrachtet, aus zwei halben Sinnfehlern zusammensetzt.

2. Der Sinn einzelner Aussagen des Primärtextes ist sinnentstellend wiedergegeben. Dieser Fehlertyp ist weitaus häufiger als der erstgenannte (sinnentstellende Wiedergabe von Relationen zwischen Aussagen).

In E 40 wird z. B. gefragt, "[...] ob für Abtreibung nicht derselbe Maßstab [wie für die Forschung mit embryonalen Stammzellen] gelten müsse". Hier ist der Versuch fehlgeschlagen, aus den Textzeilen 71-74 eine sinnvolle Makroproposition zu bilden. Von den Befürwortern der Forschung an embryonalen Stammzellen wird laut Primärtext ja gerade nicht gefordert, dass für Abtreibung derselbe Maßstab gelten solle; vielmehr führen sie die Abtreibung als Beispiel dafür an, dass der Staat die "Schutzpflicht" (Z. 27) für das Leben, auf das sich wiederum die Gegner berufen (Z. 29f.), durchaus nicht immer erfülle (ohne dass dies gegen das Gesetz gehe). Eine sinngemäß richtige Zusammenfassung hätte demnach fragen können, "[...] ob für die Forschung mit Embryonen nicht derselbe Maßstab gelten müsse wie für die Abtreibung". Da die LeserInnen von E 40 den Sinn der Primärtextstelle aus dem Exzerpt nicht erschließen können, wurde dieser Teilsatz als ganzer Sinnfehler gewertet.

Als Beispiel für einen "leichten" (halben) Sinnfehler sei die folgende Aussage aus E 32 angeführt: "Wann beginnt das menschliche Leben? fragt sich das Bundesverfassungsgericht." Hier wird nahe gelegt, das Bundesverfassungsgericht habe die entsprechende Debatte angestoßen; tatsächlich heißt es im Primärtext lediglich, "auch das Bundesverfassungsgericht [habe die für die Forschung entscheidende Frage] bisher nicht klar beantwortet" (Z. 28). Zwar ist die Gewichtung der Rolle des Bundesverfassungsgerichts in E 32 nicht korrekt, doch ist dies für das Verständnis der folgenden Aussagen nicht essentiell. Deshalb wurde hier nur ein halber Sinnfehler berechnet.

3. Im Exzerpt muss die Information des Primärtextes in einer schlüssigen Art und Weise präsentiert werden, damit die LeserInnen das Exzerpt sinnrichtig verstehen können - der Text muss kohärent sein. Als LeserInnen befragen wir nämlich, mit Nussbaumer/Sieber (1994: 162), "ein uns angebotenes sprachliches Gebilde danach, ob es uns als Rezipienten Anleitung genug ist, uns ein kohärentes Verständnis aufzubauen". Genauer betrachtet fragen wir uns also eigentlich nicht, ob ein untersuchter Text (hier: ein Exzerpt) kohärent sei, sondern "ob und wie er uns als Rezipienten anleitet und uns hilft, uns eine kohärente Textwelt im Kopf aufzubauen" (Nussbaumer/Sieber 1994: 162).

Aus dem von Nussbaumer und Sieber entwickelten "Zürcher Textanalyseraster" wurde für die vorliegende Untersuchung der Kohärenz-Aspekt der "thematischen Entfaltung" verwendet. Er erfasst die an den Text zu stellende Frage, ob "man den einzelnen Textschritt im Anschluss an die vorausgegangenen Textschritte [versteht], also "ob die Textschrittfolge folgerichtig, 'logisch' ist" (Nussbaumer/Sieber 1994: 163). Die Autoren unterscheiden zwischen einer "Texthintergrundslogik" (THL) und einer "Textvordergrundslogik" (TVL). Einem Text wird die intakte THL abgesprochen, wenn wir nicht verstehen können, was für einen Schritt eine bestimmte Textsequenz in Relation zum globalen Textziel darstellt. In solchen Fällen bricht das Verständnis, metaphorisch gesprochen, ein - es befinden sich "Brüche" im Text (ebd.: 163). Die TVL hingegen ist nicht eingehalten, wenn wir einen Text verstehen, obwohl er uns wenig dabei hilft, weil er zum Beispiel zu viele Fragen unbeantwortet lässt etc. In solchen Fällen sprechen Nussbaumer/Sieber (1994: 164) von "Sprüngen" im Text.

In der vorliegenden Untersuchung wurden "Brüche" und "Sprünge" im Text als Fehlertypen verwendet und erstere als ganze, letztere als halbe Sinnfehler gewertet. Ein Beispiel aus E 35 soll das Vorgehen illustrieren. Dort heißt es: 
"Verfassungsrechtler sehen in der derzeitigen Lage einen Gesetzesbruch. Denn wann ist der Embryo ein Mensch. Laut $\S \S 1$ und 2 des Grundgesetzes ist oberstes Gebot die Gewährleistung der Menschenwürde und das Recht auf Leben."

Diese Textstelle weist folgende Probleme auf: Abgesehen davon, dass die Aussage "Verfassungsrechtler sehen in der derzeitigen Lage einen Gesetzesbruch" so nicht stimmt, ${ }^{15}$ impliziert der Beginn des nächsten Satzes mit der Konjunktion denn, dass nun eine Begründung für das gerade Gesagte folgt. Mit der Frage, wann der Embryo ein Mensch sei, wird diese Begründung allerdings nicht eindeutig gegeben. Allerdings könnte ein Rezipient mit viel Phantasie folgern, es sei gemeint, die missliche Lage resultiere daraus, dass die erwähnte Frage so schwer zu beantworten sei. Eine/n solch phantasievolle/n LeserIn unterstellt, wurde diese Überleitung als "Textsprung", nicht als "Bruch" bewertet und deshalb ein halber Sinnfehler berechnet.

Als Satz, der auf "Denn wann ist der Embryo ein Mensch" folgt, würde man eine Antwort erwarten. Tatsächlich hat die folgende Aussage ("Laut $\S \S 1$ und $2(\ldots)$ "] mit der Frage nichts zu tun, der Zusammenhang ist nicht erkennbar. Hier liegt ein "Textbruch" vor, der mit einem ganzen Fehler bewertet wurde. Auch "Kohäsionsfehler", d. h. die falsche Anwendung von Kohäsionsmitteln wie Pronomina, Konjunktionen etc. (vgl. Nussbaumer/Sieber 1994: 165), wurden als Kohärenzfehler bewertet, allerdings nur, wenn sie zu einer sinnentstellenden Wiedergabe von Primärtextaussagen führten. Ansonsten wurden sie als "sprachliche Fehler" vernachlässigt.

\subsubsection{Gesamtbewertung der Exzerpte: der Auswertungsmaßstab}

Für jedes Exzerpt wurde ein Gesamtnote, eine Punktzahl zwischen 1 und 10, berechnet, die sich aus den drei Einzelnoten für die Kategorien "fehlende Einheiten", "Sinnfehler", "Detailliertheitsfehler" zusammensetzt. Die Einzelnoten, ebenfalls auf einer Gradierungsskala von 1-10 erstellt, flossen unterschiedlich stark in die Gesamtbewertung ein. Da der Hauptzweck des Exzerpts darin liegt, den Primärtext sinnvoll zu kürzen, sind "Sinn" und "Detailliertheit" die entscheidenden Bewertungskriterien. Die entsprechenden Fehler gingen mit je 35\% in die Gesamtnote ein. Etwas niedriger gewichtet wurden Auslassungsfehler, da der Rezipient aus einem sinnrichtigen, angemessen detaillierten Exzerpt, das im Vergleich mit dem Primärtext einige inhaltliche Lücken aufweist, eher Nutzen ziehen kann im umgekehrten Fall. Die Kategorie "fehlende Einheiten" ging mit 30\% in die Gesamtnote ein.

Für die einzelnen Kategorien wurden Punkte ("Noten") folgendermaßen vergeben:

1. Punkteverteilung für die fehlenden Einheiten:

\begin{tabular}{|l|l|}
\hline Fehlende Einheiten & Punkte \\
\hline 0 & 10 \\
\hline 1 & 8 \\
\hline 2 & 6 \\
\hline 3 & 4 \\
\hline 4 & 2 \\
\hline 5 & 0 \\
\hline
\end{tabular}

Jede Makroproposition ist eine Zusammenfassung mehrerer Informationen (da mehrerer Propositionen) der Textebene, d. h. wenn eine Makroproposition überhaupt nicht in einem Exzerpt auftaucht, gehen mehrere Informationen des Primärtextes verloren, die für die globale

\footnotetext{
15 Im Primärtext wird lediglich davon gesprochen, dass "manche Juristen [befürchten], dass der Streit über die Embryonenforschung [...] an die Fundamente der Verfassung" rühre (Z. 25f.). An keiner Stelle heißt es, es bestehe bereits ein "Gesetzesbruch". Für diese Aussage wurde denn auch ein Sinnfehler berechnet.
} 
Bedeutung des Textes wichtig sind. Deshalb muss pro fehlender Einheit selbstverständlich mehr von der Gesamtpunktzahl 10 subtrahiert werden als dies beispielsweise pro Sinnfehler der Fall ist. Wäre die Einheit im Exzerpt ausgedrückt, könnte ja auch mehr als nur ein Sinnfehler in der jeweiligen Textspanne vorhanden sein.

2. Punkteverteilung für die Sinnfehler:

\begin{tabular}{|l|l|}
\hline Sinnfehler & Punkte \\
\hline 0 & 10 \\
\hline 1 & 9 \\
\hline 2 & 8 \\
\hline 3 & 7 \\
\hline 4 & 6 \\
\hline & \\
\hline
\end{tabular}

\begin{tabular}{|l|l|}
\hline Sinnfehler & Punkte \\
\hline 5 & 5 \\
\hline 6 & 4 \\
\hline 7 & 3 \\
\hline 8 & 2 \\
\hline 9 & 1 \\
\hline 10 & 0 \\
\hline
\end{tabular}

Der Länge des Textes entsprechend durften bis zu 10 Sinnfehler gemacht werden, bevor es die Note 0 gab. Pro Sinnfehler wurde ein Notenpunkt von der Gesamtpunktzahl 10 abgezogen, wobei es bei der Punktevergabe auch entsprechende Zwischenschritte bei halben Fehlern gab (d. h., bei 1,5 Sinnfehlern wurden beispielsweise 8,5 Punkte vergeben).

3. Punkteverteilung für Fehler, die Detailliertheit betreffend:

\begin{tabular}{|l|l|}
\hline Fehler & Punkte \\
\hline 0 & 10 \\
\hline 0,5 & 9,375 \\
\hline 1 & 8,75 \\
\hline 1,5 & 8,125 \\
\hline 2 & 7,5 \\
\hline 2,5 & 6,875 \\
\hline 3 & 6,25 \\
\hline 3,5 & 5,625 \\
\hline & \\
\hline
\end{tabular}

\begin{tabular}{|l|l|}
\hline Fehler & Punkte \\
\hline 4 & 5 \\
\hline 4,5 & 4,375 \\
\hline 5 & 3,75 \\
\hline 5,5 & 3,125 \\
\hline 6 & 2,5 \\
\hline 6,5 & 1,875 \\
\hline 7 & 1,25 \\
\hline 7,5 & 0,625 \\
\hline 8 & 0 \\
\hline
\end{tabular}

Was die Detailliertheitsfehler betraf, so wurde bereits bei 8 Fehlern die Note 0 vergeben. Während man nämlich bei beispielsweise acht Sinnfehlern immer noch Teile des Primärtextes erkennen und verstehen kann, ist bei dieser Zahl von Detailliertheitsfehlern die Aufgabe deutlich verfehlt bzw. ist das Exzerpt - was die Detailliertheit anbelangt - ungenügend. Tendenziell wurde für Äußerungen, die direkt der Textebene entnommen waren, ein Fehler, für solche der ersten Makroebene ein halber Fehler berechnet. Um also tatsächlich acht Detailliertheitsfehler zu erhalten, konnten acht Sätze der Textebene einfach kombiniert werden (dies würde schon fast ein ganzes Exzerpt ergeben) - ohne sie auf irgendeine Weise zusammenzufassen -, was in jedem Fall eine ungenügende Leistung wäre (bezogen auf das Zusammenfassen der Informationen). Andererseits konnten z. B. auch 16 Sätze bzw. Makropropositionen der ersten Makroebene zu einem Text zusammengestellt werden, was in Anbetracht der sehr geringen Eigenleistung (was das Zusammenfassen betrifft) ebenfalls eine ungenügende Leistung wäre. 


\subsection{Exemplarische Auswertung des Exzerpts Nr. 24}

Im Folgenden wird die Bewertung eines Exzerpts ausführlich so dargestellt, wie sie für alle Exzerpte vorgenommen wurde. Damit soll gezeigt werden, wie die Ergebnisse im Einzelnen gewonnen wurden und wo die Hauptschwierigkeiten bei der Bewertung lagen.

Das Exzerpt wird zunächst in voller Länge abgedruckt, wobei Rechtschreib- und Zeichenfehler verbessert, alle übrigen Fehler aber wiedergegeben wurden, auch die Absätze wurden so getreu wie möglich dem handschriftlichen Bild angepasst. 
1 Forschung und Justiz befinden sich im ethischen Zwiespalt; sie können sich nicht entscheiden, ab wann das zelluläre Gebilde Embryo als Mensch bezeichnet werden kann bzw. ob dessen Tötung zur Gewinnung von Stammzellen zu rechtfertigen ist.

Auch in der Politik können die großen Parteien wegen ihrer Zerrissenheit keine einheitliche 5 Position zu diesem Thema beziehen.

Während die Befürworter der Stammzellenforschung, ungeachtet der Frage, ab wann der Embryo als Mensch gilt, die These aufstellen, dass der Staat das Leben nicht mehr schützen solle als die Natur, sprechen Gegner vom bewussten Töten von Menschen, das niemals rechtmäßig oder moralisch und ethisch vertretbar sein kann.

Ein anderes Argument für die Zulassung der Stammzellenforschung beinhaltet die Frage, ob die Zelle mehr als erwachsenes Leben geschützt werden müsse; durch die Forschung an Stammzellen würden die Chancen zur Heilung schwerer Krankheiten ja sicherlich verbessert.

Bei der Formulierung der genannten Frage, ob die Zelle mehr wert sei, muss man das ethische Hauptproblem der Stammzellendebatte betrachten: Ab wann gilt der Embryo nicht nur als "Zellhaufen" zu Forschungszwecken, sondern als Mensch mit dem Recht auf Leben?

Die Meinungen sind vielfältig; während für die einen das Leben mit der Befruchtung der Eizelle beginnt, setzen andere diesen Zeitpunkt erst bei der Nidation oder noch später an.

Begründet wird die Einstellung, dass das Leben sofort mit der Befruchtung der Eizelle beginne, dass ein individuelles Genom entstehe, in dessen weiterer Entwicklung keine Unterbrechung mehr stattfinde; das Menschwerden ist also von Anfang an gegeben.

Gegner dieser These behaupten, da ohne die Hilfe und Steuerung der Mutter kein menschliches Leben entstehen könne, die "Menschwerdung" fände erst bei der Nidation statt, wenn die Entwicklung der Embryonen mit Unterstützung des Körpers der Mutter beginnt.

Lösungs- und Kompromissvorschläge sind auf Grund von vielen verschiedenen, oft gegensätzlichen, Meinungen schwer zu finden.

Nur einen theoretischen Lösungsansatz konnte ein Neurophysiologe schon nennen: es müsste auch möglich sein, von einem wenige Tage alten Embryo Stammzellen zu gewinnen, ohne ihn zu töten.

Forschung in diese Richtung ist in Deutschland aber verboten.

30 (325 Wörter)

\section{Ermittlung der Fehleinheiten:}

Wie in 4.3.1 beschrieben, muss zur Ermittlung der fehlenden Einheiten erst einmal festgestellt werden, welche Makropropositionen überhaupt im Exzerpt vorhanden sind. Diese als vorhanden berechnete Einheiten (fett gedruckt) werden im Folgenden zusammen mit der 
jeweiligen Textstelle des Exzerpts genannt; eventuelle Erläuterungen werden direkt am Anschluss an das jeweilige Beispiel gegeben.

Vorhandene Makropropositionen:

M3;3: "Forschung und Justiz befinden sich im ethischen Zwiespalt; sie können sich nicht entscheiden, [...] ob dessen [des Embryos] Tötung zur Gewinnung von Stammzellen zu rechtfertigen ist." (Z. 1f.)

M2;9: "[...] ab wann das zelluläre Gebilde Mensch als Embryo bezeichnet werden kann." (Z. 1f.)

(M2;8 ist teilweise angedeutet, indem betont wird, dass "Forschung und Justiz" sich im "Zwiespalt" befinden bzw. in Entscheidungsnot sind; es wird also klar, dass die nun folgende Frage (M2;9) eine große Bedeutung haben muss.)

M3;3/M1;14: "Auch in der Politik können die großen Parteien wegen ihrer Zerrissenheit keine einheitliche Position zu diesem Thema beziehen." (Z. 4f.) - Dieser Satz entspricht keiner der beiden Makropropositionen ganz, da in M3;3 die Politiker nur unter anderem genannt werden, ohne dass die "großen Parteien" erwähnt sind - in M1;14 hingegen bezieht sich die "Zerrissenheit" der Politiker auf M1;13, ist also weniger allgemein.

M1;24: "Während die Befürworter der Stammzellforschung [...] die These aufstellen, dass der Staat das Leben nicht mehr schützen solle als die Natur [...]." (Z. 6ff.)

M2;24 (M3;9): "[...] sprechen Gegner vom bewussten Töten von Menschen, das niemals rechtmäßig oder moralisch und ethisch vertretbar sein kann." (Z. 8f.) - Dieser Satz entspricht keiner der Makropropositionen der Tabelle genau, aber er ist am ehesten noch mit M2;24 gleichzusetzen (obwohl er nicht eindeutig auf die Primärtextstelle Z. 64ff. zurückgeht, auf Grund derer die Makroproposition M2;24 entstand, sondern evtl. auch auf Z. 37ff.; der Satz könnte auch eine Zusammenfassung beider Stellen sein).

M2;23 (M1;37): "Ein anderes Argument für die Zulassung der Stammzellenforschung beinhaltet die Frage, ob die Zelle mehr als erwachsenes Leben geschützt werden müsse [...]." (Z. 10f.) - Hier bezieht sich der Exzerpierende auf ein Zitat aus dem Text, nämlich auf Z. 62, das so in keiner Makroproposition vorkommt; da diese Äußerung aber dieselbe Funktion übernimmt wie M2;23 (dass nämlich vorher Genanntes ${ }^{16}$ M2;21 und M2;22 - als Argument für die Embryonenforschung herangezogen wird), entspricht sie am ehesten dieser Makroproposition.

M2;22: "[...] durch die Forschung an Stammzellen würden die Chancen zur Heilung schwerer Krankheiten ja sicherlich verbessert." (Z. 11ff.) - Hiermit wird nun eine Begründung nachgeliefert für die direkt davor im Exzerpt gestellte Frage, d. h. hierdurch wird nun rechtfertigt, das Vorherige auch tatsächlich als M2;23 zu werten.

M2;8: "Bei der Formulierung der genannten Frage, ob die Zelle mehr wert sei, muss man das ethische Hauptproblem der Stammzellendebatte betrachten [...]." (Z. 14f.) - Dieser Satz entspricht zwar nicht völlig der Makroproposition M2;8; da aber auch hier noch einmal explizit ausformuliert wird, warum die nun im Exzerpt folgende Frage von Bedeutung ist, hat er zumindest dieselbe Funktion wie M2;8.

${ }^{16}$ D. h. im Zeitungsartikel vorher Genanntes bzw. in der Tabelle in Anhang 4 (cf. S. 69) vorher aufgeführte Makropropositionen. 
M2;9: "Ab wann gilt der Embryo nicht nur als 'Zellhaufen' zu Forschungszwecken, sondern als Mensch mit dem Recht auf Leben?" (Z. 15f.)

M2;10: "[...] sondern als Mensch mit dem Recht auf Leben?" (Z. 16) - Zumindest ein Teil der Informationen von M2;10, dass man nämlich als Mensch das Recht auf Leben habe, kommt hier zum Ausdruck.

M2;16: "[...] während für die einen das Leben mit der Befruchtung der Eizelle beginnt [...]" (Z. 17f.); "[...] Einstellung, dass das Leben sofort mit der Befruchtung der Eizelle beginne [...]" (Z. 19); "[...] das Menschwerden ist also von Anfang an gegeben." (Z. 21)

M1;28: "[...] dass ein individuelles Genom entstehe [...]." (Z. 20)

M1;29: "[...] in dessen weiterer Entwicklung keine Unterbrechung mehr stattfinde [...]." (Z. 20f.)

M2;12: "Gegner dieser These behaupten, da ohne die Hilfe und Steuerung der Mutter kein menschliches Leben entstehen könne [...]" (Z. 22f.); "[...] wenn die Entwicklung der Embryonen mit Unterstützung des Körpers der Mutter beginnt." (Z. 24)

M2;11: "[...] setzen andere diesen Zeitpunkt erst bei der Nidation oder noch später an" (Z. 18); "[...] die 'Menschwerdung' fände erst bei der Nidation statt." (Z. 23)

(M2;5: "Lösungs- und Kompromissvorschläge sind [...] schwer zu finden." (Z. 25f.) - Dies entspricht nur einem Teil von M2;5; es verdeutlicht die große Bedeutung von M2;3 als theoretische Lösung des "ethischen Dilemmas von Forschung, Politik und Justiz".)

M3;1 (M2;5): "Nur einen theoretischen Lösungsansatz konnte ein Neurophysiologe schon nennen [...]." (Z. 27)

M2;3: "[...] es müsste auch möglich sein, von einem wenige Tage alten Embryo Stammzellen zu gewinnen, ohne ihn zu töten." (Z. 27 ff.)

M2;6 (M3;2): "Forschung in diese Richtung ist in Deutschland aber verboten." (Z. 30)

Anhand der festgestellten Einheiten lässt sich nun sagen, dass das vorliegende Exzerpt ein eher knappes ist, d. h. es enthält vor allem Makropropositionen der zweiten und dritten Makroebene. Bei der Ermittlung der Fehleinheiten ist dies insofern von Bedeutung, als hier nur Einheiten der dritten Makroebene als fehlend berechnet würden bzw. dann auch Einheiten der zweiten Makroebene, falls es die Symmetrie erfordern würde, d. h. falls z. B. die nähere Begründung von einem Pro-, nicht aber die vom entsprechenden Contra-Argument gegeben würde.

Es fehlt hier jedoch eine Makroproposition der zweiten Ebene, nämlich die Einheit M2;13: Nur wenn gesagt wird, dass "viele frühe Embryonen [...] auf natürliche Weise verloren [gehen]" (M2;13), kann man die Äußerung, der Staat solle das Leben nicht mehr schützen als die Natur (E. 24, Z. 7f.), überhaupt verstehen. Ansonsten sind alle wichtigen Einheiten, d. h. mindestens die der dritten Makroebene, vorhanden. Allerdings weicht die Art und Weise der Gegenüberstellung der Argumente hier teilweise stark ab von der des 'idealen Exzerpts' in Kap. 2.3 (z. B. wird hier M2;14 (zusammen mit der ergänzten Einheit M2;13) der Einheit M2;24 (bzw. M3;9) gegenübergestellt; im Beispielexzerpt wurde die Einheit M2;14 der Einheit M2;15 gegenübergestellt). Diese andere Art der Darstellung ist aber ebenfalls möglich und wird nicht als falsch berechnet. Auch die Makropropositionen, die dem Exzerpt-Text zugrunde liegen könnten, unterscheiden sich teilweise von denen der Tabelle in Anhang 4, ohne dass sie aber als falsch berechnet werden müssen. Indem z. B. die Aussage, dass "Gegner vom bewussten Töten von Menschen [sprächen], das niemals [...] vertretbar sein 
kann" (E. 24, Z. 8f.) als M2;24 (bzw. zumindest eines Teils davon) gewertet wurde, wurde anerkannt, dass auch diese Formulierung eine Zusammenfassung der Textzeilen 64-70 sein kann. ${ }^{17}$

$\rightarrow$ In die Bewertung fließt ein: 1 fehlende Einheit.

\section{2. 'Detailliertheitsfehler':}

Diese Fehler werden im Folgenden nicht ihrer Art nach (Wiederholung vs. Textebene vs. erste Makroebene) dargestellt, sondern in der Reihenfolge, wie sie im Exzerpt auftauchen, da die Auswertung auch so vor sich ging, dass ein Satz nach dem anderen auf seine Detailliertheit hin untersucht wurde.

"Auch in der Politik können die großen Parteien wegen ihrer Zerrissenheit keine einheitliche Position zu diesem Thema beziehen." (Z. 4f.) - Diese Aussage entspricht in dieser ausführlichen Form keiner der Makropropositionen genau (bei 1. wurde sie zu M3;3 bzw. M1;14 gerechnet); wegen der in Abschnitt 4.3.2 erwähnten Möglichkeit, eigene Akzente zu setzen (in diesem Exzerpt wird Wert darauf gelegt, die Position der Politik als wichtigen Bereich neben "Forschung und Justiz" zu nennen), zählt sie jedoch nicht als zu detailliert.

"[...] ungeachtet der Frage, ab wann der Embryo als Mensch gilt [...]." (Z. 6f.) - Diese Äußerung ist zwar vom Sinn her richtig, sie ist aber hier völlig überflüssig und zählt (da sie nicht sehr lang ist) als leichter (= halber) Fehler.

"[...] Frage, ob die Zelle mehr als erwachsenes Leben geschützt werden müsse[...]." (Z. 11) Diese Formulierung ist zwar direkt dem Text entnommen und in keiner der Makropropositionen enthalten; da sie aber in der Art und Weise, wie sie hier im Text verwendet wird, der Makroproposition M2;23 entspricht, wird sie nicht als zu detailliert berechnet.

"Bei der Formulierung der genannten Frage, ob die Zelle mehr wert sei, muss man das ethische Hauptproblem der Stammzellendebatte betrachten: Ab wann gilt der Embryo nicht nur als 'Zellhaufen' zu Forschungszwecken, sondern als Mensch mit dem Recht auf Leben?" (Z. 14ff.) - Zwar wird auch hier teilweise nochmals die Frage wiederholt, wann der Embryo ein Mensch sei, aber in einem anderen Zusammenhang als zu Beginn des Exzerpts. Hier wird ja vor allem die Bedeutung der Frage betont, wann der Embryo das Recht auf Leben habe. Die Wiederholung ist hier so 'leicht', dass sie vernachlässigbar ist bzw. als mit inbegriffen im Fehler Z. 6f. (wo es ja um dasselbe Problem geht) angesehen werden kann.

"[...] während für die einen das Leben mit der Befruchtung der Eizelle beginnt, setzen andere diesen Zeitpunkt erst bei der Nidation oder noch später an." (Z. 17f.) - Zwar sind die Informationen, die hier gegeben werden, neu - da diese aber weiter unten (Z.19ff. bzw. Z. 22ff.), wo die "Meinungen" ausführlich wiedergegeben werden, genau so wiederholt werden, wird nun schon hier - im Voraus sozusagen - ein Fehler (wegen Wiederholung) berechnet. (Stattdessen hätte natürlich weiter unten jeweils ein leichter Fehler vergeben werden können.)

"[...] da ohne die Hilfe und Steuerung der Mutter kein menschliches Leben entstehen könne [...]" (Z. 22f.); "[...] wenn die Entwicklung der Embryonen mit Unterstützung des Körpers der Mutter beginnt." (Z. 24.) - Hierfür wird ein leichter Fehler (Wiederholung) berechnet, da in beiden Aussagen dieselbe Information gegeben wird, dass nämlich für die Entwicklung des Embryos die "Hilfe" bzw. "Unterstützung" des Körpers der Mutter nötig sei.

"Lösungs- und Kompromissvorschläge sind auf Grund von vielen verschiedenen, oft gegensätzlichen Meinungen, schwer zu finden." (Z. 25f.) - Die Aussage, dass es schwer sei, "Kompromissvorschläge" zu finden, wird nicht als zu detailliert bewertet, denn diese Information ist nicht automatisch darin impliziert, dass es "oft gegensätzliche Meinungen" gebe. Die Aussage ist sogar durchaus positiv zu bewerten, denn so wird die (große) Bedeutung des dann beschriebenen "theoretischen Lösungsansatz[es]" erst klar (auch die Makroproposition M2;5

${ }^{17}$ Zwar ist das "bewusst" hier eine etwas unglückliche Formulierung - dies kann jedoch als sprachlicher Fauxpas bewertet und somit vernachlässigt werden. 
wird dadurch teilweise impliziert). Der zweite Teil des Satzes ist jedoch überflüssig, da bereits gesagt wurde, dass "die Meinungen [...] vielfältig [sind]" (Z. 17). Dies wird, da es nur ein kurzer Einschub ist, als ein leichter Fehler (Wiederholung) berechnet.

$\rightarrow$ In die Bewertung gehen ein: 3 leichte und 1 ganzer Fehler, d. h. 2,5 Fehler (davon 2 Wiederholungsfehler).

\section{Sinnfehler:}

Um im Folgenden die vorhandenen Sinnfehler darzustellen, wird der jeweilige Ausschnitt des Exzerpts zitiert und anschließend wird besprochen, inwiefern dieser die entsprechende Aussage des ursprünglichen Textes sinnentstellt wiedergibt; außerdem wird dargelegt, wie zweifelhafte Fälle in die Bewertung mit eingingen.

"[...] ab wann das zelluläre Gebilde Embryo als Mensch bezeichnet werden kann [...]." (Z. 2f.) Wahrscheinlich würden die Gegner der embryonalen Stammzellforschung, die in dem Zeitungsartikel zitiert werden, diese nicht ganz neutrale Formulierung ("zelluläre[s] Gebilde Embryo") so nicht durchgehen lassen. Schließlich beanspruchen sie für den Embryo in jedem Stadium seiner Entwicklung die Bezeichnung "Mensch", und der Ausdruck "zelluläres Gebilde" ist zumindest leicht (ab)wertend. Allerdings weicht diese geringfügige Wertung nur so leicht vom ursprünglichen Text ab, dass hierfür kein Fehler berechnet wird. (Abgesehen davon wird die Formulierung "zelluläres Gebilde wie du und ich" auch in der Überschrift benutzt, was vielleicht auch einen Einfluss auf die Wortwahl des Exzerpierenden hatte - dort ist sie freilich provozierend gemeint.)

"Auch in der Politik können die großen Parteien wegen ihrer Zerrissenheit keine einheitliche Position zu diesem Thema beziehen." (Z.4f.) - Zwar wird im Primärtext nie ausdrücklich gesagt, dass die Politiker "zu diesem Thema" - also zu den im Exzerpt zuvor genannten Fragen - keine einheitliche Meinung besitzen; dort heißt es, "die Politiker sind im Für und Wider [...] zerrissen" (Z. 26), dies aber nur in Bezug auf die bevorstehende Entscheidung zum Stammzellimport. Da im restlichen Text jedoch klar wird, dass auch Politiker (z. B. R. Herzog) an der Diskussion beteiligt sind, kann man - obwohl hier niemals explizit gesagt wird, dass auch, was die Stammzellforschung ganz allgemein betrifft, die Politiker "zerrissen" seien - diese Aussage des Exzerpts als sinngemäß richtig durchgehen lassen. ${ }^{18}$

"Während die Befürworter der Stammzellenforschung, ungeachtet der Frage, ab wann der Embryo als Mensch gilt, die These aufstellen, dass der Staat das Leben nicht mehr schützen solle als die Natur [...]." (Z. 6ff.) - Als Sinnfehler wird an dieser Stelle nicht berechnet, dass die vorgestellte 'These' so vom Leser überhaupt nicht verstanden werden kann; wäre die unter 1. als fehlend berechnete Einheit M2;13 nämlich vorhanden, wäre dieser Satz vom Sinn her richtig. D. h., dass dieser 'Fehler' bereits bei Berechnung der fehlenden Einheit in die Bewertung mit einging. ${ }^{19}$

${ }^{18}$ Deshalb auch wurde sie ja unter 1. als Teil von M3;3 gewertet, obwohl es in M3;3 auch ganz allgemein heißt, dass sich "Politiker, Juristen und Forscher" nicht einig seien - es bleibt hier aber offen, ob die jeweiligen Gruppen auch untereinander uneinig sind.

${ }^{19}$ Fraglich ist hier deshalb lediglich, ob es richtig ist, dass diese "These" der Befürworter tatsächlich als isoliert von der Frage, "ab wann der Embryo als Mensch gilt", angesehen werden kann, wie es ja explizit in dem Exzerpt behauptet wird. Nun, im Primärtext wird die Auffassung der Befürworter, dass der Embryo erst mit der Einnistung in die Gebärmutter ein Mensch sei, direkt anschließend damit begründet, dass "die Entwicklung des Embryos von der Mutter mitgesteuert wird [...]" (Z. 33ff.) (diese Begründung wird mit "da" (Z. 34) eingeleitet). Der nun folgende Satz ("Zwei Dritter aller Embryonen [...]", Z. 35f.) schließt ohne irgendeine Überleitung an, d. h. dass eigentlich vom Rezipienten davon ausgegangen werden könnte, dass auch dieser sich noch mit der eben genannten 'Hauptfrage' beschäftigt bzw. eine weitere Begründung dafür ist schließlich wird nichts Gegenteiliges angezeigt. Allerdings ist nicht eindeutig klar, in welcher Beziehung dieser Satz zu der zuvor genannten 'Hauptfrage' steht. Zusammen mit dem nächsten Satz ("Herzog fragte, ob der Staat [...]", Z. 36f.) wird zwar geklärt, dass Herzog die Tatsache, dass Embryonen auf natürliche Weise verloren gehen, als 'Pro-Argument' für die Forschung heranzieht; es wird allerdings nicht eindeutig gesagt, dass er damit auch begründen will, dass der Embryo erst bei der Nidation als Mensch angesehen werden 
"Bei der Formulierung der genannten Frage, ob die Zelle mehr wert sei [...]." (Z. 14) - Gemeint ist hier wohl, dass es fraglich sei, ob auch schon der "Zellhaufen" vor der Nidation als Mensch betrachtet werden sollte (also "mehr wert" sei als irgendeine beliebige "Zelle"); da dies von einem Leser/einer Leserin im Normalfall allerdings so nicht erschlossen werden kann, wird hierfür ein leichter Fehler berechnet (da die Aussage des ursprünglichen Textes zwar vom Sinn her nicht ganz richtig wiedergegeben wurde, dieser Fehler jedoch nicht sehr schwerwiegend ist und das Verständnis des restlichen Exzerpts nicht beeinträchtigt).

"Ab wann gilt der Embryo nicht nur als Zellhaufen zu Forschungszwecken [...]." (Z. 15f.) Hier taucht ein ähnliches Problem wie beim ersten oben besprochenen Punkt auf: die gestellte Frage ist so nicht neutral; das "Hauptproblem der Stammzellendebatte" (Z. 15) kann so nicht formuliert werden. Für die Gegner gilt der Embryo ja eben nie "nur als 'Zellhaufen'", sondern immer, während seiner ganzen Entwicklung, "als Mensch mit dem Recht auf Leben". Deshalb ist die Formulierung "ab wann" hier sinnentstellend; auch hierfür wird ein leichter Fehler berechnet.

"[...] dass ein individuelles Genom entstehe, in dessen weiterer Entwicklung keine Unterbrechung mehr stattfinde [...]." (Z. 20f.) - Zwar ist hier wohl das Richtige gemeint (dass nämlich ein Genom entstehe, das die weitere Entwicklung des Embryos bestimmt, bei der es "keine Unterbrechung" mehr gibt), d. h. dies könnte theoretisch als ein 'Leichtsinns-Grammatik' oder '-Kohäsionsfehler' vernachlässigt werden; da die Grenze zwischen eindeutig als 'Leichtsinnsfehler' erkennbaren und anderen Fehlern allerdings fließend ist, wird in solchen Fällen jeder Fehler berechnet, d. h. auch hierfür wird ein leichter Fehler berechnet.

$\rightarrow$ In die Bewertung fließen ein: 3 leichte, d. h. 1,5 Sinnfehler.

\section{Gesamtbewertung:}

Für das Kriterium der Fehleinheiten werden 8 Notenpunkte (1 Fehleinheit) vergeben, für das der Sinnfehler 8,5 Notenpunkte (1,5 Fehler), und die Detailliertheit betreffend erhält das Exzerpt 6,875 Punkte (2,5 Fehler). Die daraus entstehende Gesamtnote - berechnet nach dem Maßstab aus Abschnitt 4.3.4 - ist 7,78.

\subsection{Darstellung der Ergebnisse der textlinguistischen Analyse}

Zwei der 33 abgegebenen Exzerpte (E 41 und 49) konnten nicht ausgewertet werden, weil jeweils nur zwei bis drei Sätze geschrieben wurden und deshalb nicht genügend Material für eine sinnvolle Auswertung vorhanden war (zu eventuellen Ursachen vgl. Abschnitt 4.6.2, Fragebogen 2, besonders Fragen 7 und 12). Die verbleibenden 31 Exzerpte wurden so ausgewertet, wie das anhand des Beispiels in Abschnitt 4.4 vorgeführt wurde.

In der folgenden Auswertungstabelle wird nicht ersichtlich, dass es in den unterschiedlichen Exzerpten teilweise ähnliche Fehler gab oder dass es unterschiedliche Fehler gab, die jeweils auf eine Stelle des Primärtextes zurückgeführt werden konnten. Besonders deutlich war dies an der Primärtextstelle Z. 59-61 zu beobachten ("Der Lebensschutz kann unter bestimmten Bedingungen eingeschränkt werden, etwa in Notwehrsituationen, für Herzog ein Grund für die Zulässigkeit der Forschung an Embryonen, der Suche nach Heilung von schweren Krankheiten."). Auffällig viele SchülerInnen hatten Schwierigkeiten, diese Stelle sinnrichtig wiederzugeben: Nur 14 SchülerInnen gaben sie richtig wieder, 13 waren dazu nicht in der Lage, und 4 SchülerInnen erwähnten sie gar nicht. Dieses Ergebnis deutet darauf hin, dass das

müsse. Deshalb gilt die Aussage des Exzerpts, dass Herzogs "These" (Z. 7) "ungeachtet" (Z. 6) dieser Frage aufgestellt werde, nicht als falsch. - Aus demselben Grund wurden auch bei der Erstellung der Tabelle 1 die entsprechenden Makropropositionen nicht etwa folgendermaßen gebildet: M2;11: "Befürworter haben die Auffassung, dass die Entwicklung des Embryos zum Menschen erst mit der Einnistung in die Gebärmutter beginnt"; M2;12: "M2;11, da die Entwicklung des Embryos von der Mutter abhängig ist" und M2;13: "M2;11, da viele frühe Embryonen auf natürliche Weise verloren gehen". 
Problem mit größter Wahrscheinlichkeit in der Schwierigkeit der Textstelle selbst begründet liegt. Das wiederum wirft die Frage auf, ob bei der Auswertung die - offensichtliche Komplexität einzelner Textstellen berücksichtigt werden sollte. Unseres Erachtens ist das nicht erforderlich, denn das Verstehen komplexer Aussagen soll ja ein Faktor für das Gelingen eines Exzerptes sein, und dass der Text als ganzer für die Aufgabe durchaus angemessen war, haben wir dargelegt. Nicht zuletzt ist die Frage, ob eine Textstelle nun als besonders schwierig einzuordnen ist oder nicht, selbstverständlich nicht objektiv zu beantworten. ${ }^{20}$

Die folgende Tabelle 2 stellt die Ergebnisse der Auswertung von 31 Exzerpten dar, davon 12 aus dem Deutsch-Leistungskurs (LK), 19 aus dem Grundkurs (GK). Für jedes Exzerpt werden im Folgenden die jeweiligen Fehlerzahlen und die entsprechenden Notenpunkte für jede der drei Kategorien sowie die Gesamtbewertung angegeben.

${ }^{20}$ Als wie schwierig nach dem subjektiven Empfinden der SchülerInnen einzelne Stellen des Primärtextes beurteilt wurden, stellen wir bei der Auswertung von Fragebogen 2 in Abschnitt 4.6.2 (Fragen 4-6) dar. 


\begin{tabular}{|c|c|c|c|c|c|c|c|c|c|}
\hline $\begin{array}{l}\text { Exz.- } \\
\text { Nr. }\end{array}$ & $\begin{array}{l}\text { Fehlende } \\
\text { Einheiten }\end{array}$ & Note & $\begin{array}{l}\text { Sinn- } \\
\text { Fehler }\end{array}$ & $\begin{array}{c}\underline{\text { davon }} \\
\text { Kohärenzfehler }\end{array}$ & $\underline{\text { Note }}$ & $\begin{array}{l}\text { 'Detailliert- } \\
\text { heitsfehler' }\end{array}$ & $\begin{array}{c}\text { davon } \\
\text { Wdh.-fehler } \\
\end{array}$ & Note & $\frac{\text { GESAMT- }}{\text { NOTE }}$ \\
\hline \multicolumn{10}{|l|}{ LK: } \\
\hline 4 & 0 & 10 & 2 & 0,5 & 8 & 2 & 0 & 7,5 & 8,4 \\
\hline 11 & 4 & 2 & 5,5 & 0 & 4,5 & 0 & 0 & 10 & 5,7 \\
\hline 23 & 2 & 6 & 3 & 2 & 7 & 5 & 0,5 & 3,75 & 5,6 \\
\hline 24 & 1 & 8 & 1,5 & 0 & 8,5 & 2,5 & 2 & 6,875 & 7,8 \\
\hline 35 & 3 & 4 & 9,5 & 4,5 & 0,5 & 3,5 & 0 & 5,625 & 3,3 \\
\hline 36 & 4 & 2 & 5 & 3 & 5 & 4,5 & 1,5 & 4,375 & 3,9 \\
\hline 40 & 3 & 4 & 2,5 & 0 & 7,5 & 0 & 0 & 10 & 7,3 \\
\hline 45 & 0 & 10 & 2 & 0,5 & 8 & 1,5 & 0 & 8,125 & 8,6 \\
\hline 67 & 1 & 8 & 8,5 & 3 & 1,5 & 3 & 0 & 6,25 & 5,1 \\
\hline 70 & 1 & 8 & 6,5 & 0,5 & 3,5 & 5,5 & 0,5 & 3,125 & 4,7 \\
\hline 71 & 1 & 8 & 2,5 & 1 & 7,5 & 4,5 & 0 & 4,375 & 6,6 \\
\hline 89 & 2 & 6 & 1,5 & 1 & 8,5 & 0,5 & 0 & 9,375 & 8,1 \\
\hline \multicolumn{10}{|l|}{ GK: } \\
\hline 31 & 0 & 10 & 3 & 0,5 & 7 & 4,5 & 0 & 4,375 & $\mathbf{7 , 0}$ \\
\hline 32 & 3 & 4 & 8 & 0,5 & 2 & 5 & 0 & 3,75 & 3,2 \\
\hline 33 & 3 & 4 & 2,5 & 0 & 7,5 & 1,5 & & 8,125 & 6,7 \\
\hline 34 & 1 & 8 & 3 & 0,5 & 7 & 1,5 & 0 & 8,125 & 7,7 \\
\hline 39 & 1 & 8 & 4,5 & 0,5 & 5,5 & 3 & 0 & 6,25 & 6,5 \\
\hline 42 & 0 & 10 & 1 & 0 & 9 & 5 & 0,5 & 3,75 & 7,5 \\
\hline 43 & 2 & 6 & 3 & 1,5 & 7 & 5 & 0 & 3,75 & 5,6 \\
\hline 44 & 1 & 8 & 2,5 & 0,5 & 7,5 & 7 & 0 & 1,25 & 5,5 \\
\hline 46 & 0 & 10 & 2 & 0 & 8 & 3,5 & 0 & 5,625 & 7,8 \\
\hline 47 & 0 & 10 & 2,5 & 0 & 7,5 & 4 & 0 & 5 & 7,4 \\
\hline 48 & 1 & 8 & 3 & 1,5 & 7 & 6 & 0,5 & 2,5 & 5,7 \\
\hline 50 & 2 & 6 & 4,5 & 0,5 & 5,5 & 3 & 0,5 & 6,25 & 5,9 \\
\hline 51 & 1 & 8 & 3,5 & 1 & 6,5 & 4 & 0 & 5 & 6,4 \\
\hline 52 & 2 & 6 & 7 & 0,5 & 3 & 4,5 & 0,5 & 4,375 & 4,4 \\
\hline 53 & 1 & 8 & 5,5 & 1 & 4,5 & 7,5 & 0 & 0,625 & 4,2 \\
\hline 55 & 4 & 2 & 2,5 & 0,5 & 7,5 & 0,5 & 0 & 9,375 & 6,5 \\
\hline 63 & 1 & 8 & 2,5 & 0,5 & 7,5 & 6 & 0 & 2,5 & 5,9 \\
\hline 64 & 1 & 8 & 4 & 0,5 & 6 & 3 & 0 & 6,25 & 6,7 \\
\hline 68 & 3 & 4 & 3 & 0 & 7 & 5,5 & 0 & 3,125 & 4,7 \\
\hline
\end{tabular}

Tabelle 2: Gesamtauswertung der Exzerpte

\begin{tabular}{|c|c|}
\hline Notendurchschnitt aller 31 Exzerpte & 6,14 \\
\hline Notendurchschnitt LK & 6,26 \\
\hline Notendurchschnitt GK & 6,06 \\
\hline Notendurchschnitt Schülerinnen & 6,13 \\
\hline Notendurchschnitt Schüler & 6,14 \\
\hline
\end{tabular}

\section{Tabelle 3: Notendurchschnitte}

Man sieht in Tabelle 3, dass der Notendurchschnitt im LK etwas höher ist als der im GK, was angesichts der höheren Wochenstundenzahl des Faches (fünf vs. drei) nicht überrascht. Im Gegenteil: die Durchschnittsnote der LK-SchülerInnen hätte nach unserer Erwartung deutlicher über der der GK-SchülerInnen liegen sollen. 
Mit einer Bewertung von 3,2 wurde das schlechteste Exzerpt von einer Schülerin des GKs geschrieben, das beste Exzerpt (Note: 8,6) schrieb eine Schülerin des LKs. Des weiteren erhielt niemand im GK eine Bewertung, die besser als 8,0 war, drei SchülerInnen des LKs aber hatten eine solch gute Bewertung. Wenn man die beiden Kurse nach dem (sehr guten oder sehr schlechten) Abschneiden einzelner SchülerInnen vergleicht, zeigt sich also, dass die besten Exzerpierergebnisse im LK erreicht wurden, das schlechteste im GK. Allerdings ist im Ganzen betrachtet in keiner der beiden Gruppen ein einheitliches Bild festzustellen. So finden sich auch bei den 12 Exzerpten der LK-SchülerInnen immerhin zwei Bewertungen, die schlechter als 4,0 (nämlich 3,3 und 3,9) sind; die restlichen Bewertungen wurden zwischen 4,0-4,9 (einmal), zwischen 5,0-5,9 (dreimal), zwischen 6,0-6,9 (einmal), zwischen 7,0-7,9 (zweimal) und zwischen 8,0-8,9 (dreimal) vergeben. Was die Noten der GK-Gruppe betrifft, so sieht die Verteilung hier folgendermaßen aus: Bewertungen wurden zwischen 3,0-3,9 (einmal), 4,0-4,9 (dreimal), 5,0-5,9 (fünfmal), 6,0-6,9 (fünfmal) und 7,0-7,9 (fünfmal) vergeben.

Es gibt in der GK-Gruppe also mehr "mittelgute" Bewertungen: 10 von 19 SchülerInnen des GKs haben eine Bewertung, die zwischen 5,0 und 6,9 liegt; dieses Noten-Mittelfeld ist beim LK mit nur vier von zwölf SchülerInnen deutlich schwächer besetzt. Um ein detaillierteres und aussagekräftigeres Bild der Exzerpierkenntnisse zu erhalten, werden sie im Folgenden außerdem nach Fehlerarten aufgesplittet betrachtet:

Was die Sinnfehler betrifft, so ergibt sich ein allgemeiner Fehlerdurchschnitt von 3,79 Fehlern bzw. 6,21 Notenpunkten; der LK hat im Durchschnitt 4,17 Fehler $(5,83$ Notenpunkte), im GK sind es 3,55 Fehler (6,45 Notenpunkte). Hier schneiden die SchülerInnen des Grundkurses also ein wenig besser ab als ihre KollegInnen aus dem Leistungskurs.

Die durchschnittliche Anzahl von Fehleinheiten ist 1,58, was 6,84 Notenpunkten entspricht; bei der Aufteilung nach Kursen ist auch hier wieder der LK ein wenig besser: bei 1,42 Fehleinheiten ist die durchschnittliche Note hier 7,16. Die SchülerInnen des GKs haben durchschnittlich 1,83 Fehleinheiten, was der Note 6,33 entspricht.

Was die Detailliertheit der Exzerpte anbelangt, so haben die SchülerInnen im Durchschnitt 3,63 Fehler, was 5,46 Notenpunkten entspricht; der LK hat im Schnitt 2,71 Fehler (Note: 6,62), der GK 4,21 Fehler (Note: 4,74). Was die Detailliertheit ihrer Exzerpte anbelangt, sind also die SchülerInnen des Leistungskurses deutlich besser als die des Grundkurses.

Zusammenfassend zu diesen drei Fehlerarten lässt sich feststellen, dass die Exzerpte der SchülerInnen des LKs weniger Detailliertheitsfehler enthalten als die der SchülerInnen des GKs. Wenn man davon ausgeht, dass die LK-SchülerInnen die erfahreneren Schreiber/innen bzw. "Zusammenfasser/innen" sind, dann passt dieses Ergebnis sehr gut zu den Ergebnissen der von Keseling (1993) vorgestellten empirischen Untersuchungen (TEIL A, 1.2), dass nämlich erfahrenere SchreiberInnen besser zwischen wichtigen und unwichtigen Informationen unterscheiden können sowie besser kurze Zusammenfassungen produzieren können (13). Zwar bedeuten weniger Detailliertheitsfehler nicht unbedingt, dass das Exzerpt wirklich kürzer ist. Die Auswertungsmethode lässt selbstverständlich auch zu, dass längere, detaillierte Exzerpte geschrieben werden, die keine Detailliertheitsfehler enthalten (dies war ja schließlich Grundvoraussetzung für die Methode). Dennoch gilt für die Schülerexzerpte tendenziell, dass die eher kürzeren Exzerpte weniger Detailliertheitsfehler aufweisen als die eher längeren. Dass die SchülerInnen des GKs mehr Detailliertheitsfehler haben, passt ebenfalls gut zu dem bei Keseling (1993) präsentierten Ergebnis, dass unerfahrene SchreiberInnen oft eine einfache "copy-delete-strategy" anwenden (14) - solch ein Übernehmen wörtlicher Textpassagen führte ja ganz besonders zu Detailliertheitsfehlern. Zu der geringeren Anzahl von "Detailliertheitsfehlern" beim LK passt auch der - im Vergleich zum GK - etwas höhere Wert bei den fehlenden Einheiten (1,83 zu 1,42 Fehleinheiten). Es ist 
durchaus vorstellbar, dass bei knapperen Exzerpten eher einmal etwas 'Wichtiges' weggelassen wird als bei sehr detaillierten Exzerpten. Selbstverständlich müssen wirklich gute und erfahrene Exzerpt-SchreiberInnen knappe Exzerpte schreiben können, die dennoch alle wichtigen Details enthalten - aber die SchülerInnen des LKs scheinen denen des GKs in dieser Hinsicht nicht so sehr überlegen zu sein - dies könnte man aus diesem Ergebnis schließen -, dass sie knappere Exzerpte, die von der Detailliertheit her besser sind, schreiben können, ohne dass dabei auch teilweise etwas mehr Information verloren geht (aber beide Kurse sind hinsichtlich der fehlenden Informationen ja allgemein durchaus nicht schlecht).

Insgesamt betrachtet sowie was die Detailliertheit der Exzerpte anbelangt, sind also die SchülerInnen des LKs besser als ihre KollegInnen aus dem GK, die aber wiederum weniger Fehler bei den Fehleinheiten sowie bei den Sinnfehlern haben. Allgemein lassen sich in beiden Kursen bessere und schlechtere Exzerpierkenntnisse feststellen - dass es aber insgesamt immerhin sieben SchülerInnen gibt, deren Note unter 5,0 liegt und dass es nur drei SchülerInnen gibt, die mit einer Note über 8,0 relativ gut abschneiden bzw. überhaupt keine SchülerInnen, die besser als 8,6 sind, zeigt deutlich, dass man hier auf keinen Fall von allgemein guten Exzerpierfähigkeiten sprechen kann. ${ }^{21}$ Bei so wenig wirklich guten Ergebnissen (wenn man einmal davon ausgeht, dass die drei Ergebnisse über 8 "wirklich gut" sind) könnte man vielleicht sogar vermuten, dass die Fähigkeit der drei Besten eher auf eine besondere Begabung auf Seiten der SchülerInnen zurückgeht als auf tatsächlich hierauf ausgerichteten Unterricht. Doch es war ja nicht das Ziel dieser Studie, Überlegungen darüber anzustellen, inwiefern die Schule die Methode des Exzerpierens genügend in den Unterricht integriert (solche Überlegungen wären allerdings auf Grund der gefundenen Ergebnisse nicht fehl am Platze!). Es sollte vielmehr gezeigt werden, wie die Exzerpierfähigkeiten der SchülerInnen tatsächlich aussehen - und da diese sehr gemischt, im Großen und Ganzen aber nur bei den allerwenigsten "gut" zu nennen sind, lässt sich folgende Aussage treffen:

Die Ergebnisse, die die Auswertung von 31 Exzerpten lieferte, zeigen, dass es falsch wäre, wenn die Hochschulen davon ausgingen, dass StudienanfängerInnen sämtliche für das wissenschaftliche Arbeiten nötigen Exzerpierkenntnisse von der Schule mitbringen.

Für das Gros der an der Studie beteiligten SchülerInnen gilt, dass ihre Fähigkeiten nicht ausreichen würden, um an der Hochschule ohne weiteren Unterricht gute Exzerpierergebnisse zu erzielen.

\subsection{Zur Selbsteinschätzung der ProbandInnen: ein Fragebogenerhebung}

\subsubsection{Fragebogen 1}

Fragebogen 1 (vgl. Anhang 2), der den SchülerInnen einige Tage vor der Durchführung der Exzerpieraufgabe vorgelegt wurde, sollte unter anderem Aufschluss darüber geben, inwieweit sich die SchülerInnen der Wichtigkeit des Exzerpierens bewusst sind bzw. inwiefern sie es in ihrem Schulalltag nutzen (vgl. Abschnitt 4.1). Im Folgenden stellen wir einige der Ergebnisse überwiegend in Tabellenform dar und geben kurze Interpretationshinweise. Die Tabellen nennen jeweils die Anzahl der SchülerInnen, die die jeweilige Frage beantwortet haben, sowie die Zahlen (absolut und in \%) der jeweiligen Antworten. In Klammern werden die Zahlen der Leistungskurs-SchülerInnen separat angegeben, weil diese Gruppe aufgrund des intensiveren Deutschunterrichts besonders interessiert.

${ }^{21}$ Dass außerdem zwei Schüler den Exzerpiervorgang sogar ganz abgebrochen haben, lässt bei ihnen ebenfalls eher auf "schlechtere" als auf "bessere" Fähigkeiten schließen. 
Ausnahmslos alle SchülerInnen gaben an, das Exzerpieren von Texten schon einmal geübt zu haben. Auf Befragen der LehrerInnen ergab sich, dass die Befragten unter "Üben" jede Art von Praxis verstanden hatten, also nicht etwa nur die explizite methodische Anleitung zum Exzerpieren. Unter den Fächern, in denen das Exzerpieren geübt worden war, liegen Deutsch (29 Nennungen, davon 10 LK), Geschichte (17/9) und Englisch (10/2) mit Abstand vorn.

Die Frage "Hast du schon einmal - unabhängig davon, ob es im Schulunterricht schon geübt wurde oder nicht - einen Text exzerpiert (z. B. zu Hause)?" wurde wie folgt geantwortet:

\begin{tabular}{|c|c|c|c|}
\hline & Ja & Nein & Insgesamt \\
\hline Anzahl - absolut & $29(12)$ & $4(0)$ & $33(12)$ \\
\hline Anteil (in \%) & $87,9(100)$ & $12,1(0)$ & $100(100)$ \\
\hline
\end{tabular}

Tabelle 4: Schon einmal exzerpiert? (in Klammern: LK)

Auch unabhängig von Schulunterricht schein das Exzerpieren also gängige Praxis zu sein, allerdings sagen diese Zahlen weder etwas über die Häufigkeit aus, mit der das Exzerpieren praktiziert wird, noch darüber, wie die SchülerInnen "Exzerpieren" genau auffassen (z. B. könnte das reine Herausschreiben von Informationen aus einem Text hier durchaus mitgemeint sein).

Die dritte Frage, ob sich die SchülerInnen schon einmal auf eine Klassenarbeit vorbereitet hatten, indem sie wichtige Texte exzerpiert hatten, ergab folgende Zahlen:

\begin{tabular}{|c|c|c|c|c|}
\hline & Ja & Nein & Weiß nicht & Insgesamt \\
\hline Anzahl - absolut & $29(12)$ & $2(0)$ & $2(0)$ & $33(12)$ \\
\hline Anteil (in \%) & $87,9(100)$ & $6,1(0)$ & $6,1(0)$ & $100(100)$ \\
\hline
\end{tabular}

Tabelle 5: Exzerpieren für Klassenarbeit I (in Klammern: LK)

Alle SchülerInnen, die überhaupt schon einmal einen Text exzerpiert hatten, haben dies auch schon einmal für eine Klassenarbeit getan. Die funktionale Relevanz des Exzerpierens steht also für die SchülerInnen außer Frage, wobei, wie gesagt, hier vermutlich ein weiter Begriff von "Exzerpt" zugrunde liegt.

Dieses Einschätzung kommt auch direkt in den Antworten auf die Frage zum Ausdruck, ob die SchülerInnen fänden, dass das Exzerpieren von Texten ihnen dabei helfe, diese besser zu verstehen:

\begin{tabular}{|c|c|c|c|c|}
\hline & Ja & Nein & Weiß nicht & Insgesamt \\
\hline Anzahl - absolut & $31(11)$ & $2(1)$ & $0(0)$ & $33(12)$ \\
\hline Anteil (in \%) & $93,9(91,7)$ & $6,1(8,3)$ & $0(0)$ & $100(100)$ \\
\hline
\end{tabular}

Tabelle 6: Exzerpieren und Textverständnis (in Klammern: LK)

Die folgenden drei Fragen zielen auf die Probleme, die die SchülerInnen mit dem Exzerpieren haben; die erste lautete: "Hattest du schon einmal Probleme, einen Text zu exzerpieren (z. B. weil dieser zu kompliziert war)?"

\begin{tabular}{|c|c|c|c|c|}
\hline & Ja & Nein & Weiß nicht & Insgesamt \\
\hline Anzahl - absolut & $28(11)$ & $3(1)$ & $2(0)$ & $33(12)$ \\
\hline Anteil (in \%) & $84,8(91,7)$ & $9,1(8,3)$ & $6,1(0)$ & $100(100)$ \\
\hline
\end{tabular}

Tabelle 7: Probleme beim Exzerpieren? (in Klammern: LK) 
Der Anteil der $J a$-Antworten auf diese Frage spricht für sich. Die folgende Frage diente der genaueren Erhebung der Häufigkeit, mit der Probleme beim Exzerpieren auftreten: "Wie oft hast du Probleme beim Exzerpieren von Texten?":

\begin{tabular}{|c|c|c|c|c|c|}
\hline & Sehr oft & Oft & Manchmal & Selten & Insgesamt \\
\hline Anzahl - absolut & $1(0)$ & $3(0)$ & $20(9)$ & $6(2)$ & $30(11)$ \\
\hline Anteil (in \%) & $3,3(0)$ & $10(0)$ & $66,7(81,8)$ & $20(18,2)$ & $100(100)$ \\
\hline
\end{tabular}

Tabelle 8: Häufigkeit der Probleme (in Klammern: LK)

24 von 30 SchülerInnen (80\%) haben manchmal oder öfter Probleme beim Exzerpieren, wobei die SchülerInnen des LK etwas weniger häufig von Problemen berichten als die des GK. Schon diese Zahl allein würde - ganz abgesehen von den Ergebnissen der Hauptuntersuchung - Grund genug sein, sich darüber Gedanken zu machen, ob es gerechtfertigt ist, dass man an der Hochschule in der Regel davon ausgeht, die StudienanfängerInnen würden bereits über eine hinreichende Exzerpierfähigkeit verfügen. Da die Anforderungen an das Exzerpieren mit Sicherheit im Studium anwachsen, kann davon ausgegangen werden, dass sich auch die dadurch entstehenden Probleme - falls ihnen nicht konsequent begegnet wird - eher vergrößern.

Die nächste Frage zielte auf die Qualität der Probleme: "Als wie schlimm empfindest du diese Probleme?" Die Antwort war auf einer fünfstufigen Skala von 1 (= "überhaupt nicht schlimm") bis 5 (= "sehr schlimm") durch Ankreuzen zu geben:

\begin{tabular}{|c|c|c|c|c|c|c|}
\hline & 1 & 2 & 3 & 4 & 5 & Insgesamt \\
\hline Anzahl - absolut & $2(2)$ & $12(4)$ & $9(2)$ & $6(3)$ & $1(0)$ & $30(11)$ \\
\hline Anteil (in \%) & $6,7(18,2)$ & $40(36,4)$ & $30(18,2)$ & $20(27,3)$ & $3,3(0)$ & $100(100)$ \\
\hline
\end{tabular}

Tabelle 9: Qualität der Probleme (in Klammern: LK)

Mehr als die Hälfte aller Befragten (53,3\%) empfindet die Probleme beim Exzerpieren als "mittelschlimm" bis "sehr schlimm" (= '3'-'5'). Die drei SchülerInnen, die zuvor angegeben hatten, sie hätten 'oft' Probleme, kreuzten bei dieser Frage '3', '3' und '4' an (Mittelwert: 3,3). Der Schüler, der angab, "sehr oft" Probleme zu haben, empfindet diese Probleme sogar als sehr schlimm $(=' 5 ')$. Die SchülerInnen, die bei Frage 6 angaben, "manchmal" Probleme zu haben, gaben bei dieser Frage im Schnitt 2,7 an, der Mittelwert der sechs SchülerInnen, die "selten" Probleme haben, ist hier 2,17. Es besteht also eine Tendenz, dass SchülerInnen, die öfter Probleme haben, diese auch als schlimmer zu empfinden scheinen als solchen, die weniger oft Probleme haben.

Im Weiteren war noch einmal die Vorbereitung von Klassenarbeiten Thema: "Ich halte das Exzerpieren von Fachtexten (z. B. Biologie, Geschichte etc.) für eine gute Möglichkeit, sich auf Klassenarbeiten vorzubereiten." Vorgegeben war eine Skala von eins ('stimme überhaupt nicht zu') bis fünf ('stimme voll zu'):

\begin{tabular}{|l|c|c|c|c|c|c|c|}
\hline & 1 & 2 & 3 & 4 & 5 & Weiß nicht & Insgesamt \\
\hline Anzahl - absolut & $0(0)$ & $2(1)$ & $8(2)$ & $9(2)$ & $13(7)$ & $1(0)$ & $33(12)$ \\
\hline Anteil (in \%) & $0(0)$ & $6,1(8,3)$ & $24,2(16,7)$ & $27,3(16,7)$ & $39,4(58,3)$ & $3,0(0)$ & $100(100)$ \\
\hline
\end{tabular}

Tabelle 10: Exzerpieren für Klassenarbeit II (in Klammern: LK)

Bemerkenswerte 66,7\%, also zwei Drittel aller Befragten, bewerteten die oben gemachte Aussage mit '4' oder '5', sind also ("ziemlich" oder "ganz") der Ansicht, dass man sich durch Exzerpieren von Fachtexten gut auf Klassenarbeiten vorbereiten kann. Betrachtet man den Deutsch-Leistungskurs getrennt, erhöht sich dieser Anteil sogar auf 75\%. Nur zwei von 33 SchülerInnen (LK: einer von 12) bewerteten die Aussage mit '2' - kein/e SchülerIn wies sie völlig ab. Ein Großteil aller SchülerInnen schätzt das Exzerpieren also als durchaus 
bedeutsame Arbeitsmethode zur Vorbereitung von Klassenarbeiten - und dies trotz der Probleme, die sie damit haben (vgl. Fragen 5-7). Es deutet sich hier also ein Ungleichgewicht an zwischen der Bedeutung, die selbst die SchülerInnen dem Exzerpieren schon zumessen, einerseits (vgl. Fragen 4 und 8), und den Problemen, die sie damit haben und denen wohl kaum oder gar nicht entgegen gewirkt wird, andererseits. Dies erinnert an das von Ehlich/Steets $\left(2000^{b}\right)$ an der Hochschule gefundene Ergebnis, dass Einschätzung der Bedeutsamkeit der Technik und die Lehrpraxis stark auseinander klaffen. Dass sich dieser Zustand an der Schule schon andeutet, spricht nun besonders dafür, ihm (spätestens) an der Hochschule konsequent entgegenzuwirken - nur so kann verhindert werden, dass die Studierenden in solch alten Mustern verbleiben und sich die Probleme verfestigen.

Im Rahmen einer Studie, die herausfinden will, mit welchen Exzerpierkenntnissen und -fähigkeiten Studierende das erste Semester beginnen, ist es besonders interessant zu erfahren, wie viele und wer von den SchülerInnen die Absicht haben, zu studieren. Zunächst wurde deshalb gefragt: "Möchtest du später einmal studieren?":

\begin{tabular}{|c|c|c|c|c|}
\hline & Ja & Nein & Weiß ich jetzt noch nicht & Insgesamt \\
\hline Anzahl - absolut & $19(6)$ & $5(1)$ & $9(5)$ & $33(12)$ \\
\hline Anteil (in \%) & $57,6(50,0)$ & $15,2(8,3)$ & $27,3(41,7)$ & $100(100)$ \\
\hline
\end{tabular}

Tabelle 11: Studieren? (in Klammern: LK)

Wer diese Frage mit "ja" oder "weiß ich jetzt noch nicht" beantwortet hatte, sollte in der folgenden Frage ein Wunschstudienfach oder eine Studienrichtung angeben, die dann von uns in 4 Gruppen zusammengefasst wurden. In der folgenden Tabelle sind den Gruppen die jeweiligen Exzerptnummern zugeordnet:

\begin{tabular}{|c|c|c|c|c|c|c|}
\hline & $\begin{array}{c}\text { Geistesw.- } \\
\text { schaftlicher } \\
\text { Bereich }\end{array}$ & $\begin{array}{c}\text { Sozialw.- } \\
\text { schaftlicher } \\
\text { Bereich }\end{array}$ & $\begin{array}{c}\text { Naturw.- } \\
\text { schaftlicher } \\
\text { Bereich }^{22}\end{array}$ & $\begin{array}{c}\text { Internationales } \\
\text { Medien }\end{array}$ & $\begin{array}{c}\text { Weiß noch } \\
\text { nicht }\end{array}$ & Insgesamt \\
\hline Anzahl - abs. & $7(2)$ & $3(3)$ & $3(0)$ & $4(1)$ & $11(5)$ & $28(12)$ \\
\hline Anteil (in \%) & $25(16,7)$ & $10,7(25)$ & $10,7(0)$ & $14,3(8,3)$ & $39,3(41,7)$ & $100(100)$ \\
\hline Exzerpt-Nr. & $\begin{array}{c}89,70,64, \\
33,46,39,\end{array}$ & $11,36,40$ & $68,34,32$ & $4,50,31,47$ & $71,23,24$, & $28(12)$ \\
& 52 & & & $35,55,43$, & \\
\hline
\end{tabular}

Tabelle 12: Wunschfachrichtung (in Klammern: LK) und Exzerpt-Nummer

Das Verhältnis von Wunschstudium und Exzerptqualität lässt sich auf der Grundlage dieser Zuordnung sehr differenziert betrachten. Wir fassen hier nur die wichtigsten Ergebnisse zusammen: Die beste durchschnittliche Exzerptqualität erreichte die Gruppe derer, die ihren Studienwunsch schon kennen (und auch studieren möchten), aber sicher keine Naturwissenschaften studieren wollen ( $\mathrm{N}=12$; Durchschnitt: 6,51), dicht gefolgt von einer Gruppe, die sich von der vorigen Gruppe nur dadurch unterscheidet, dass hier auch solche SchülerInnen dabei sind, die noch nicht sicher wissen, ob sie tatsächlich studieren wollen $(\mathrm{N}=14$; Durchschnitt: 6,45). Ebenfalls wieder nur geringfügig schlechter waren die SchülerInnen, die sicher studieren wollen, allerdings keine Naturwissenschaften (bzw. teilweise noch keinen Studienwunsch angegeben konnten), $(\mathrm{N}=16$; Schnitt: 6,43). Den vierten Platz erreichte die Gruppe all derer, die sicher studieren wollen ( $\mathrm{N}=19$; Schnitt: 6,24).

\footnotetext{
22 Einschl. Ingenieursstudiengänge, z. B. Maschinenbau.

23 In diese Rubrik wurden Studiengänge wie "Internationales Kulturmanagement", "Internationale BWL" o. Ä. eingeordnet.
} 


\subsubsection{Fragebogen 2}

Wir kommen nun zur Auswertung des Fragebogens, der sich auf die Exzerpieraufgabe bezieht. Die erste Frage lautete: "Wie schwierig empfandest du den Text, den du exzerpiert hast? Ordne ihn in einer Schwierigkeitsskala von 1 (= überhaupt nicht schwierig) bis 5 (= sehr schwierig) ein." (Frage 1)

\begin{tabular}{|c|c|c|c|c|c|c|}
\hline & 1 & 2 & 3 & 4 & 5 & Insgesamt \\
\hline Anzahl - abs. & $0(0)$ & $10(6)$ & $15(6)$ & $8(0)$ & $0(0)$ & $33(12)$ \\
\hline Anteil (in \%) & $0(0)$ & $30,3(50,0)$ & $45,5(50,0)$ & $24,2(0)$ & $0(0)$ & $100(100)$ \\
\hline
\end{tabular}

Tabelle 13: Schwierigkeit des Primärtextes (in Klammern: LK)

Angesichts der teilweise relativ schlechten Exzerpierleistungen ist auffallend, dass keine/r der SchülerInnen den Primärtext als "sehr schwierig" beurteilte. Schwierigkeitsgrad '4' wies ihm ein knappes Viertel der SchülerInnen zu, darunter kein/e LK-SchülerIn. Die meisten ProbandInnen $(75,8 \%)$ empfanden ihn als "nicht sehr schwierig" bis "mittelschwierig". Wenn wir unterstellen, dass die ProbandInnen in ihrer Selbsteinschätzung bei dieser Frage nicht durchweg irren, müssen wir schließen, dass weniger der Text selbst Probleme bereitete als vielmehr die mangelnde Kenntnis, wie ein gutes Exzerpte anzufertigen sei.

Gibt es einen Zusammenhang zwischen Exzerpierleistung und Einschätzung der Schwierigkeit des Primärtextes? Unter den 8 SchülerInnen, die den Wert '4' vergaben, ist auch einer der Schüler, deren Exzerpt nicht gewertet werden konnte (E 41). ${ }^{24}$ Die verbleibenden 7 Exzerpte erhielten eine durchschnittliche Bewertung von 6,02, liegen also nur knapp unter dem Mittelwert aller Exzerpte $(6,14)$. Auch sind von diesen 7 SchülerInnen, die den Wert '4' vergaben, den Primärtext also als schwieriger empfanden als die übrigen Schülerinnen, 6 nicht identisch mit den SchülerInnen, die die schlechtesten Noten erhielten. Umgekehrt formuliert: Nur ein Exzerpt (E 68), das zu den sieben schlechtesten gehört, wurde von einem Schüler geschrieben, der den Text mit Schwierigkeitsgrad '4' einschätzte. D. h., es lässt sich keine Tendenz dahingehend feststellen, dass diejenigen, die den Primärtext als am schwierigsten empfanden, schlechtere Noten hatten als diejenigen, die den Text als nicht so schwierig empfanden.

Mit den folgenden Fragen (2-10) sollte herausgefunden werden, worin genau die Schwierigkeiten des Primärtextes bzw. die Schwierigkeiten beim Exzerpieren bestanden. Die entsprechenden Aussagen des Fragebogens sollten auf einer Skala von 1 ("stimme überhaupt nicht zu") bis 5 ("stimme voll zu") bewertet werden. Die erste Aussage lautet: "Einmaliges Lesen reicht aus, um den Text vollständig zu verstehen." (Frage 2)

\begin{tabular}{|c|c|c|c|c|c|c|}
\hline & 1 & 2 & 3 & 4 & 5 & Insgesamt \\
\hline Anzahl - abs. & $11(5)$ & $9(2)$ & $8(3)$ & $5(2)$ & $0(0)$ & $33(12)$ \\
\hline Anteil (in \%) & $33,3(41,7)$ & $27,3(16,7)$ & $24,2(25)$ & $15,2(16,7)$ & $0(0)$ & $100(100)$ \\
\hline
\end{tabular}

Tabelle 14: Einmaliges Lesen? (in Klammern: LK)

Wie bei einem relativ komplexen Text zu erwarten, stimmte niemand der Aussage völlig zu. Gut $60 \%$ der SchülerInnen bewerteten sie entweder mit '1' oder '2', waren also der Ansicht, dass man den Text mehr als einmal lesen musste, um ihn vollständig zu verstehen. Immerhin gut $15 \%$ bzw. fünf SchülerInnen vergaben allerdings den Wert '4', stimmten also der Aussage, wenn auch nicht voll, zu. Von diesen 5 SchülerInnen hatten zuvor drei die Schwierigkeit des Textes mit '2' bewertet, fanden ihn also nicht schwierig. Dieses Antwortverhalten ist also konsistent. Nicht so bei zwei anderen SchülerInnen, die hier den Wert '4' vergaben: Sie hatten

${ }^{24}$ Man könnte daraus schließen, dass der Schüler das Exzerpt nicht aus Unlust abgebrochen hat, sondern weil er die Aufgabe als zu schwierig empfand. Ein solcher Schluss ist selbstverständlich spekulativ. 
den Primärtext zuvor mit '4' bewertet, also als schwierig eingestuft. Man könnte spekulieren, dass sich diese beiden SchülerInnen als besonders kompetente Textrezipienten betrachteten.

Die nächste Aussage lautete: "Ich fand es schwierig, diesen Text zu exzerpieren." (Frage 3)

\begin{tabular}{|c|c|c|c|c|c|c|}
\hline & 1 & 2 & 3 & 4 & 5 & Insgesamt \\
\hline Anzahl - abs. & $1(0)$ & $13(8)$ & $15(4)$ & $3(0)$ & $1(0)$ & $33(12)$ \\
\hline Anteil (in \%) & $3,0(0)$ & $39,4(66,7)$ & $45,5(33,3)$ & $9,1(0)$ & $3,0(0)$ & $100(100)$ \\
\hline
\end{tabular}

Tabelle 15: Exzerpieren schwierig? (in Klammern: LK)

Gut $42 \%$ der SchülerInnen fanden es überhaupt nicht ('1') oder nicht sehr ('2') schwierig, den Primärtext zu exzerpieren - ein erstaunlich hoher Prozentsatz, wenn man auch hier wieder die teilweise schlechte Bewertung der Exzerpte bedenkt. Es hat hier den Anschein, als würden die SchülerInnen ihre eigene Exzerpierfähigkeit höher einschätzen als sie tatsächlich ist.

Interessant ist die Verknüpfung mit Frage 1, nämlich: Fanden diejenigen, die den Primärtext insgesamt als schwierig empfanden, auch das Exzerpieren des Textes als schwierig bzw. fanden diejenigen, die den Primärtext weniger schwierig fanden, auch das Exzerpieren leichter? Eine ausführliche Auswertung würde den Rahmen dieses Artikels sprengen, zusammenfassend aber lässt sich sagen: Fast alle SchülerInnen (30 von 33) fanden den Primärtext entweder gleich schwer oder schwieriger als das Exzerpieren, nur 3 SchülerInnen ordneten das Exzerpieren des Textes als schwieriger ein. Die Aufgaben des Textverständnisses und des Exzerpierens wurden also im Vergleich für diesen Text von der Mehrheit nicht etwa so gewichtet, dass die Exzerpieraufgabe das größere Problem dargestellt hätte. Möglicherweise spiegelt auch dieser Befund eine gewisse Unterschätzung der Exzerpieraufgabe wieder.

Die folgenden Aussagen zielten auf eine Präzisierung dessen, was am Primärtext als schwierig empfunden wurde:

"Die Sprache/die Ausdrucksweise des Textes war schwer verständlich." (Frage 4)

\begin{tabular}{|l|c|c|c|c|c|c|}
\hline & 1 & 2 & 3 & 4 & 5 & Insgesamt \\
\hline Anzahl - abs. & $7(4)$ & $18(6)$ & $5(2)$ & $2(0)$ & $1(0)$ & $33(12)$ \\
\hline Anteil (in \%) & $21,2(33,3)$ & $54,5(50,0)$ & $15,2(16,7)$ & $6,1(0)$ & 3,0 & $100(100)$ \\
\hline
\end{tabular}

Tabelle 16: Sprache schwierig? (in Klammern: LK)

Mit der sprachlichen Form des Textes hatten die meisten SchülerInnen keine größeren Probleme: über 75\% hatten keine ('1') bis wenig ('2') Probleme. (Die beiden Fremdwörter, die auch unserer Erwartung nach eventuell Probleme hätten bereiten können - "Nidation" und "Zäsuren" - wurden in beiden Kursen ja erläutert; vgl. Abschnitt 4.1).

"Die Argumentation der Autorin war schwer zu verstehen." (Frage 5)

\begin{tabular}{|c|c|c|c|c|c|c|}
\hline & 1 & 2 & 3 & 4 & 5 & Insgesamt \\
\hline Anzahl - abs. & $8(3)$ & $17(7)$ & $8(2)$ & $0(0)$ & $0(0)$ & $33(12)$ \\
\hline Anteil (in \%) & $24,2(25,0)$ & $51,5(58,3)$ & $24,2(16,7)$ & $0(0)$ & $0(0)$ & $100(100)$ \\
\hline
\end{tabular}

Tabelle 17: Argumentation schwierig? (in Klammern: LK)

Auch was die Argumente betraf, gab der Großteil (gut 75\%) aller SchülerInnen an, diese seien leicht ('1') bzw. ziemlich leicht ('2') zu verstehen gewesen. Knapp ein Viertel bewertete die Verständlichkeit der Argumente als 'mittelschwer' ('3'). Die Argumente an sich scheinen also von den meisten verstanden worden zu sein (bzw. die Selbsteinschätzung war in dieser Hinsicht wieder positiv). 
"Der Textaufbau war schwer nachvollziehbar (= die Art und Weise, wie die Argumente aufeinander folgten oder auseinander entwickelt wurden)." (Frage 6)

\begin{tabular}{|c|c|c|c|c|c|c|}
\hline & 1 & 2 & 3 & 4 & 5 & Insgesamt \\
\hline Anzahl - abs. & $4(0)$ & $8(5)$ & $10(3)$ & $10(3)$ & $1(1)$ & $33(12)$ \\
\hline Anteil (in \%) & $12,1(0)$ & $24,2(41,7)$ & $30,3(25,0)$ & $30,3(25,0)$ & $3,0(8,3)$ & $100(100)$ \\
\hline
\end{tabular}

Tabelle 18: Textaufbau schwierig? (in Klammern: LK)

Es war zu erwarten, dass der Textaufbau durchaus Probleme bereiten konnte. Der Artikel folgt ja keinem einfachen dialektischen Muster, indem beispielsweise jedes Pro- mit dem entsprechenden Contra-Argument präsentiert wird oder einer Präsentation aller ProArgumente die Darstellung aller Contra-Argumente folgt. Tatsächlich wurde von der SchülerInnen der Aufbau als der problematische Aspekt des Primärtextes beurteilt. Immerhin 11 von 33 Schüler/innen, also ein Drittel gab an, der obigen Aussage ziemlich ('4') oder völlig ('5') zuzustimmen. Ein knappes Drittel (30,3\%) gab an, mittelschwere Probleme mit dem Textaufbau gehabt zu haben, ein gutes Drittel (36,3\%) bewertete die Aussage mit '1' oder '2'. Die SchülerInnen hatten also nicht so sehr damit Probleme, einzelne Argumente der Autorin zu verstehen, sondern mehr mit dem Aufbau des Textes bzw. damit, wie die Argumentation entfaltet wurden.

Die nächsten Aussagen bezogen sich auf Teilaspekte des Exzerpierens:

"Es fiel mir schwer, den Text zu kürzen." (Frage 7)

\begin{tabular}{|c|c|c|c|c|c|c|}
\hline & 1 & 2 & 3 & 4 & 5 & Insgesamt \\
\hline Anzahl - abs. & $4(1)$ & $15(7)$ & $7(2)$ & $5(2)$ & $2(0)$ & $33(12)$ \\
\hline Anteil (in \%) & $12,1(8,3)$ & $45,5(58,3)$ & $21,2(16,7)$ & $15,2(16,7)$ & $6,1(0)$ & $100(100)$ \\
\hline
\end{tabular}

Tabelle 19: Text kürzen schwierig? (in Klammern: LK)

Einer der entscheidenden Zwecke des Exzerpierens ist es ja, einen Text zu kürzen. Gut ein Fünftel aller SchülerInnen (21,2\%) stimmte der Aussage, dies sei ihnen beim vorliegenden Text schwer gefallen, mit '4' bzw. '5' zu. Auffällig ist hierbei, dass die beiden Schüler, die der Aussage (als einzige!) voll zustimmten, es also sehr schwer fanden, den Text zu kürzen, auch diejenigen waren, deren Exzerpt nicht gewertet werden konnte, da es vorzeitig abgebrochen wurde. Man könnte hier durchaus einen Zusammenhang vermuten: Vielleicht fiel es ihnen so schwer, den Text zu kürzen, dass sie - zumindest unter anderem - deswegen gar die ganze Aufgabe als unlösbar empfanden? Gut ein weiteres Fünftel der SchülerInnen fand diese Aufgabe "mittelschwer" (bewertete sie mit '3'), und knapp 58\% fiel das Kürzen gar nicht ('1') oder nicht sehr ('2') schwer. Insgesamt gaben also über $42 \%$ an, "mittelschwere" bis "schwere" Probleme ('3'-'5') beim Kürzen gehabt zu haben.

Ob und wie sehr sich diese Probleme, die die SchülerInnen beim Kürzen des Textes hatten, in ihren Exzerpten niederschlugen (also ob sie es trotz Problemen geschafft haben, ihr Exzerpt knapp zu halten oder ob sie wegen dieser Probleme viele 'Detailliertheitsfehler' hatten, und umgekehrt), soll im Folgenden kurz betrachtet werden: Die fünf SchülerInnen, die der Aussage mit '4' zugestimmt hatten (die Exzerpte der beiden, die hier '5' angaben, konnten ja nicht gewertet werden), hatten - was die Bewertung der Detailliertheit ihrer Exzerpte betraf einen Durchschnitt von 4,5 (Gesamtdurchschnitt des Kriteriums Detailliertheit 5,46). Der Schnitt der sieben SchülerInnen, die '3' angaben, beträgt 4,8, ist also erwartungsgemäß besser als der derjenigen SchülerInnen, die die größten Probleme empfanden. Der Schnitt der 15 SchülerInnen, die mit ' 2 ' bewerteten, beträgt 5,83. Tendenziell scheinen sich also die empfundenen Probleme mit dem Kürzen des Textes auch tatsächlich dementsprechend als 'Detailliertheitsfehler' in den Exzerpten niedergeschlagen zu haben. 
"Es fiel mir schwer, einzelne Argumente des Textes herauszuarbeiten." (Frage 8)

\begin{tabular}{|c|c|c|c|c|c|c|}
\hline & 1 & 2 & 3 & 4 & 5 & Insgesamt \\
\hline Anzahl - abs. & $4(1)$ & $9(4)$ & $18(7)$ & $1(0)$ & $1(0)$ & $33(12)$ \\
\hline Anteil (in \%) & $12,1(8,3)$ & $27,3(33,3)$ & $54,5(58,3)$ & $3,0(0)$ & $3,0(0)$ & $100(100)$ \\
\hline
\end{tabular}

Tabelle 20: Argumente herausarbeiten schwierig? (in Klammern: LK)

Während sich Frage 5 auf den Primärtext bezog (ob nämlich in diesem Text einzelne Argumente gut verstanden werden konnten), liegt der Fokus dieser Frage gleichermaßen auf dem Primärtext und auf dem Exzerpieren bzw. dem zu erstellenden Exzerpt. Herausgefunden werden sollte, inwiefern es (ausgelöst durch eventuelle Schwierigkeiten mit dem Text/Textaufbau) den SchülerInnen schwer fiel, für ihre Exzerpte passende, einzelne Argumente herauszuarbeiten.

Erschwerend konnte sich hier beispielsweise auswirken, dass die Argumente teilweise so ineinander verstrickt waren, dass es nicht einfach war, hieraus eine globale Struktur zu abstrahieren und einzelne, für das Exzerpt wichtige Argumente herauszuisolieren. Immerhin gut $60 \%$ gaben an, dass dieses Herausarbeiten einzelner Argumente ihnen "mittelschwer" bis "sehr schwer" ('3'-'5') gefallen sei. Weniger Probleme damit ('1'-'2') hatten 39,4\%.

Beides - die Probleme den Textaufbau betreffend (Frage 6) sowie die Schwierigkeit, einzelne Argumente herauszuarbeiten (Frage 8) - spiegelt sich auch in den Exzerpten wider: So übernahmen viele (teilweise wörtlich) einfach nur einzelne Äußerungen oder Argumente aus dem ursprünglichen Text, die isoliert vielleicht durchaus verstanden wurden (vgl. Frage 5); diese wurden aber teilweise in einer solchen Weise miteinander bzw. mit dem restlichen Exzerpt verknüpft (vgl. Kohärenzfehler), dass sich erstens zeigt, dass keine für das Exzerpt sinnvollen Argumente (auf einer globaleren Ebene als die des ursprünglichen Textes) herausgearbeitet wurden, und dass man zweitens daraus schließen kann, dass zumindest teilweise der Zusammenhang der Argumente des ursprünglichen Textes nicht wirklich verstanden wurde (Textaufbau).

"Das Exzerpieren hat mir zu einem besseren Textverständnis verholfen als zuvor reines Lesen des Textes." (Frage 9)

\begin{tabular}{|c|c|c|c|c|c|c|}
\hline & 1 & 2 & 3 & 4 & 5 & Insgesamt \\
\hline Anzahl - abs. & $2(1)$ & $1(0)$ & $8(5)$ & $13(3)$ & $9(3)$ & $33(12)$ \\
\hline Anteil (in \%) & $6,1(8,3)$ & $3,0(0)$ & $24,2(41,7)$ & $39,4(25,0)$ & $27,3(25,0)$ & $100(100)$ \\
\hline
\end{tabular}

Tabelle 21: Exzerpieren und Textverständnis (in Klammern: LK)

Was das bessere Textverständnis durch Exzerpieren ganz allgemein anbelangt, so waren 93,9\% aller Schüler/innen (bzw. 31 von 33) der Meinung, dies treffe zu (vgl. Fragebogen 1, Frage 4). Zwei Drittel der SchülerInnen stimmten auch hier der entsprechenden Aussage mit '4' oder '5' zu. Knapp ein Viertel bewertete die Aussage mit '3', und nicht einmal 10\% bewerteten sie mit '1' oder '2'. Dies bedeutet, dass die große Mehrheit der SchülerInnen sich hier (i. e. beim bzw. nach dem tatsächlichen Verfassen von Exzerpten) einer für sie als SchülerInnen sehr großen Bedeutung des Exzerpierens durchaus bewusst war: dass nämlich dadurch ein besseres Textverständnis erlangt werden kann.

"Durch meinen (Deutsch-)Unterricht habe ich mich für diese Aufgabe ausreichend vorbereitet gefühlt." (Frage 10)

\begin{tabular}{|c|c|c|c|c|c|c|}
\hline & 1 & 2 & 3 & 4 & 5 & Insgesamt \\
\hline Anzahl - abs. & $2(0)$ & $10(2)$ & $13(6)$ & $7(3)$ & $1(1)$ & $33(12)$ \\
\hline Anteil (in \%) & $6,1(0)$ & $30,3(16,7)$ & $39,4(50)$ & $21,2(25)$ & $3,0(8,3)$ & $100(100)$ \\
\hline
\end{tabular}

Tabelle 22: Auf das Exzerpieren vorbereitet? (in Klammern: LK) 
Hier wurde (analog zur ersten Frage des ersten Fragebogens) absichtlich offen gelassen, ob die Vorbereitung für "diese Aufgabe" durch den Deutschunterricht oder durch andere Unterrichtsfächer erfolgte, da ja auch bei Frage 1 des ersten Fragebogens mehrere Unterrichtsfächer genannt wurden, in denen mit den SchülerInnen das Exzerpieren geübt wurde. Knapp $40 \%$ aller Schüler/innen bzw. 50\% des Deutsch-Leistungskurses bewerteten diese Aussage mit '3', fanden sich also durch den Unterricht "mittelgut" vorbereitet. Immerhin knapp ein Viertel fand sich ziemlich gut ('4') oder sehr gut ('5') vorbereitet, gut $36 \%$ fanden sich überhaupt nicht ('1') oder nicht sehr vorbereitet ('2'). Es ergibt sich hier also kein einheitliches Bild. Die Verteilung ist ziemlich gestreut, wobei der größte Teil sich "mittelgut" vorbereitet fühlte.

"Hattest du Vorkenntnisse im Themengebiet des Textes?" (Frage 11)

\begin{tabular}{|c|c|c|c|c|}
\hline & Ja & Nein & Ein bisschen & Insgesamt \\
\hline Anzahl - absolut & $4(2)$ & $7(3)$ & $21(7)$ & $32(12)$ \\
\hline Anteil (in \%) & $12,5(16,7)$ & $21,9(25,0)$ & $65,6(58,3)$ & $100(100)$ \\
\hline
\end{tabular}

Tabelle 23: Vorkenntnisse vom Thema? (in Klammern: LK)

Schließlich war es wichtig zu erfahren, wie viel Vorkenntnisse die SchülerInnen im Themengebiet hatten, da größere Vorkenntnisse normalerweise ein schnelleres Einordnen von neuen Informationen in vorhandenes Wissen ermöglichen bzw. diese sinnvoll(er) gespeichert und wohl auch wiedergegeben werden können (vgl. auch Keseling 1993: 6). Dies könnte sich also durchaus in den Ergebnissen der Exzerpte wiederspiegeln.

Fast zwei Drittel $(65,6 \%)$ aller SchülerInnen gab an, teilweise Vorkenntnisse gehabt zu haben. Unter diesen 21 SchülerInnen waren auch die beiden, deren Exzerpt nicht bewertet werden konnte - der Durchschnitt der verbleibenden 19 SchülerInnen bei den Bewertungen der Exzerpte war 6,22. Der Schnitt derjenigen, die angaben, keine Vorkenntnisse gehabt zu haben $(\mathrm{N}=7)$ war 5,65, was deutlich schlechter ist. Diejenigen, die angaben, gute Vorkenntnisse gehabt zu haben $(\mathrm{N}=4)$, hatten einen Gesamtdurchschnitt von 7,02. Tatsächlich erreichten also, was die Durchschnitte betrifft, diejenigen die besten Exzerptbewertungen, die die meisten Vorkenntnisse hatten $(\mathrm{N}=4)$; "mittelgute" Ergebnisse wurden von denjenigen erzielt, die mittlere Vorkenntnisse hatten $(\mathrm{N}=19)$ und diejenigen ohne jegliche Vorkenntnisse hatten im Schnitt die schlechtesten Exzerpte $(\mathrm{N}=7)$.

"Falls du Probleme beim Erstellen des Exzerpts hattest (z. B. Zeitdruck, Text zu schwierig etc.), könntest du diese bitte kurz erläutern?" (Frage 12)

Mit Absicht wurde diese Frage offen gestellt, um den SchülerInnen die Möglichkeit zu geben, die Probleme aus ihrer Sicht zu benennen. In der Auswertung wurden dann ähnlich formulierte Probleme zusammengefasst (absolute Zahlen der Nennung in Klammern).

Was die äußeren Bedingungen der Schreibaufgabe betrifft, so wurde "Zeitmangel" am häufigsten als Problem empfunden; auch fehlende Motivation (1) und Konzentrationsprobleme (2) wurden beklagt.

Der Primärtext wurde als "zu kompliziert, zu wissenschaftlich" beschrieben (2); andere empfanden ihn vor allem deshalb als sehr komplex, weil die Argumente "über den ganzen Text verteilt" gewesen seien (4). Auch fehlendes Hintergrundwissen auf Grund des "sehr speziellen Themas" wurde beklagt (4); deswegen sei es beispielsweise schwer gefallen, die Argumente unterschiedlich zu gewichten.

Was nun das Exzerpieren selbst betraf, so war das am häufigsten genannte Problem das, eigene Worte zu finden (6). In eine ähnliche Richtung geht die Klage anderer SchülerInnen, sie hätten den Text lieber in Stichworten oder in Tabellenform zusammengefasst (2). Außerdem gab es Probleme damit, Informationen herauszustreichen bzw. die im Text gege- 
benen Informationen zu selektieren - das Kürzen des Textes fiel also schwer (4). Eng damit verbunden ist die Unsicherheit, auch wirklich alles Wichtige erkannt zu haben (2). Auch formale Unsicherheiten wurden deutlich (2): genannt wurden u.a. Schwierigkeiten mit der Struktur des Exzerpts, mit dem zu verwendenden Tempus und mit der Zitierweise. Erwähnt wurde auch das Problem, "die eigene Meinung zu ignorieren" (1); andere allerdings nannten als Problem die Schwierigkeit, eine eigene Meinung zu finden bzw. Stellung zu nehmen (2).

Bei den von den SchülerInnen genannten Problemen fällt auf, dass viele der Schwierigkeiten nicht bestehen müssten bzw. beseitigt werden könnten, wenn grundlegende Vorgehensweisen beim Exzerpieren bekannt wären bzw. wenn die SchülerInnen durch Üben eine gewisse Sicherheit erlangt hätten. Selbstverständlich trifft dies nicht auf alle Probleme zu. So kann fehlendes Hintergrundwissen beispielsweise auch bei geübteren SchreiberInnen ein Problem beim Exzerpieren darstellen; allerdings würde auch hier entsprechende Übung zu größerer Sicherheit und einem souveräneren Umgang mit dem Problem verhelfen. Die meisten der genannten Probleme können aber tatsächlich durch gezieltes 'Lehren' einzelner Aspekte des Exzerpierens verringert oder gar beseitigt werden. So können formale Anforderungen klar dargelegt, Formulierungen geübt werden (Problem der eigenen 'Wortfindung'); auch der Umgang mit wissenschaftlichen bzw. allgemein komplexen Texten kann geübt werden. Übung und Routine machen es einfacher, auch bei unterschiedlichen Texten die wichtigsten Informationen herauszuselektieren (Problem des Kürzens/Selektierens), außerdem können so die Arbeitsschritte immer schneller ausgeführt werden (Problem des Zeitmangels). Die genannten Probleme sind somit vorwiegend von solcher Art, dass sie durch effektives Lehren beseitigt werden könnten. Zugleich wird deutlich, dass es notwendig ist, die Arbeitsmethode des Exzerpierens dort zu lehren, wo sie gebraucht wird: Selbstverständlich zum einen in der Schule, zum anderen müssen aber auch die Hochschulen genau bei diesen Problemen ansetzen, wenn sie wollen, dass ihre Studierenden diese Arbeitsmethode beherrschen.

Zum Abschluss wurden zwei allgemeine Fragen gestellt, die nicht direkt mit dem zuvor geschriebenen Exzerpt in Verbindung standen:

"Hältst du das Exzerpieren allgemein für eine bedeutsame Arbeitsmethode für dich als Schüler/Schülerin?" (Frage 13)

Auch hier sollten die SchülerInnen wieder nach einer Skala zwischen 1 (= 'überhaupt nicht bedeutsam') und 5 (= 'sehr bedeutsam') entscheiden. Die Verteilung sah folgendermaßen aus:

\begin{tabular}{|l|c|c|c|c|c|c|}
\hline & 1 & 2 & 3 & 4 & 5 & Insgesamt \\
\hline Anzahl - abs. & $0(0)$ & $2(0)$ & $7(3)$ & $9(5)$ & $14(4)$ & $32(12)$ \\
\hline Anteil (in \%) & $0(0)$ & $6,3(0)$ & $21,9(25,0)$ & $28,1(41,7)$ & $43,8(33,3)$ & $100(100)$ \\
\hline
\end{tabular}

Tabelle 24: Exzerpieren - bedeutsam? (in Klammern: LK)

Während nur 2 SchülerInnen das Exzerpieren für nicht sehr bedeutsam ('2') hielten, waren beachtliche 23 (9) oder 71,9\% der SchülerInnen der Ansicht, das Exzerpieren sei eine ziemlich ('4') bis sehr bedeutsame ('5') Arbeitsmethode für sie. Hier zeigt sich also ein weiteres Mal (vgl. auch F. 9 sowie F. 4 und 8 von Fragebogen 1), dass die SchülerInnen sich der Bedeutung der Methode durchaus bewusst sind; hält man sich aber die Probleme vor Augen, die einerseits bei den Exzerptbewertungen deutlich wurden, andererseits von den SchülerInnen selbst genannt wurden (F. 12 bzw. F. 7 und 8), dann wird deutlich, dass sie die Methode wohl nicht so nutzen können, wie es anbetracht ihrer Bedeutung eigentlich angebracht wäre. Auch hier wird wieder deutlich, dass ein Bedarf herrscht für ein (intensiveres) Lehren dieser Methode (an der Schule bzw. an der Hochschule). Dass der Großteil der SchülerInnen die Methode für so wichtig hält, lässt eventuell außerdem darauf schließen, dass die nötige Motivation zum Üben etc. durchaus vorhanden wäre. 
Nach dieser allgemeinen Einschätzung durch die SchülerInnen sollte die letzte Frage Aufschluss darüber geben, wie sehr das Exzerpieren vom Einzelnen/von der Einzelnen als Arbeitsmethode tatsächlich genutzt wurde:

"Falls überhaupt, inwiefern ist das Exzerpieren für dich von Bedeutung (z. B. Vorbereitung auf das Abitur)?" (Frage 14)

Bei der Auswertung der Antworten auf diese Frage wurde deutlich, dass sie sehr unterschiedlich aufgefasst wurde. Einige nannten als Antworten nämlich Gründe dafür, warum das Exzerpieren ganz allgemein wichtig sei. So waren gängige Antworten, man könne dadurch Texte im Allgemeinen besser verstehen, das Exzerpieren sei eine wichtige Arbeitsmethode für das spätere Studium, es sei "überhaupt im Leben" wichtig, weil man "immer fähig dazu sein [müsse], einen Text zu analysieren und zu verstehen"; man erhalte durch das Zusammenfassen von Texten mit eigenen Worten außerdem ein gutes Sprachgefühl und mehr Sprachsicherheit etc.

Insofern Antworten tatsächlich darauf eingingen, wie das Exzerpieren im persönlichen Schulalltag genutzt wird, sollen diese im Folgenden einfach wiedergegeben werden (ohne dass sie beispielsweise kategorisiert werden). Quantitativ ausgewertete Antworten zu der Frage, ob (und wie sehr) die SchülerInnen das Exzerpieren allgemein als wichtig empfanden, wurden ja bereits in Frage 13 dargelegt. (Zahlen in Klammern geben auch hier wieder die Anzahl der SchülerInnen an, die eine jeweilige Antwort gegeben haben.)

Drei SchülerInnen antworteten, sie würden das Exzerpieren überhaupt nicht nutzen; einer fand es zur Vorbereitung von Klassenarbeiten zwar "eigentlich schon bedeutsam", aber eben zu zeitaufwändig, ein anderer hielt es nur dann für sinnvoll, wenn man sein Exzerpt mit dem vergleichen könne, "was andere aus demselben Text herausgearbeitet haben". Dieser letzte Punkt mag zu Übungszwecken ja durchaus sinnvoll sein, verkennt abgesehen davon allerdings die wichtigsten Bedeutungen des Exzerpierens. Ein weiterer Schüler verwies auf die "vielen Exzerpte", die in "Abitrainingsbüchern oder anderen Schülerhilfen" schon vorhanden seien; deshalb würde er es nicht für nötig halten, selbst Exzerpte anzufertigen. Solche 'Hilfsliteratur' ist zweifellos eine wichtige Lernhilfe, sie aber als Ersatz für selbst angefertigte Exzerpte zu sehen, verkennt natürlich wieder wichtige Aspekte des Exzerpierens, z. B. den textverarbeitenden Charakter. Obwohl sich sehr viele SchülerInnen, wie deutlich wurde, der großen Bedeutung des Exzerpierens durchaus bewusst sind, zeigen diese Antworten teilweise, dass noch mehr darauf aufmerksam gemacht werden muss, wie wichtig das Exzerpieren tatsächlich ist.

Gut die Hälfte der SchülerInnen (17) gab an, die Methode des Exzerpierens persönlich zu nutzen, einige von ihnen (5) erwähnten allerdings ergänzend, dass sie 'Exzerpieren' hierbei nicht so verstünden wie bei der zuvor durchgeführten Aufgabe, sondern dass sie eher nur Stichworte aus dem Text herausschreiben bzw. teilweise einfach die wichtigsten Textsätze abschreiben würden. Was die persönliche Nutzung (unabhängig von der jeweiligen Definitionsweise) betraf, so wurden die Vorbereitung auf das Abitur (6), die Vorbereitung auf Klassenarbeiten (explizit genannt wurden hier die Fächer Geschichte, Deutsch, Latein und Biologie) (6), die Arbeit im Unterricht (3), die Vorbereitung für Referate (1) oder mehrere dieser Punkte genannt. Fünf SchülerInnen (von den 17) gaben an, das Exzerpieren zu nutzen, erläuterten aber nicht näher, wie.

\section{$5 \quad$ Fazit}

Wir haben in dieser Studie zunächst plausibel zu machen versucht, dass das Exzerpieren eine große Bedeutung für Studierende aller Fachrichtungen hat. Diese Einschätzung steht in deutlichem Kontrast zur geringen Beachtung, die die Textsorte Exzerpt im Lehrangebot der 
Hochschulen im Allgemeinen erfährt. Da das Exzerpieren aber, so wurde ebenfalls deutlich, ein äußerst komplexer Textreproduktionsprozess ist, ist es nicht verwunderlich, dass Studierende Probleme damit haben, "gute" Exzerpte zu erstellen (vgl. Dittmann et al. 2003, Ehlich/Steets $2000^{b}$ ). Sie sind dabei folglich oft auf ihre Intuition und ihre Kenntnisse, die sie aus der Schule mitbringen, angewiesen. Wie die Exzerpierkenntnisse von SchülerInnen am Ende ihrer Schullaufbahn tatsächlich aussehen, war die Fragestellung der durchgeführten empirischen Untersuchung, die in Abschnitt 4 beschrieben wurde.

Anhand der Analyse und Bewertung von 31 Exzerpten (12 von SchülerInnen eines DeutschLeistungskurses, 19 von solchen eines Deutsch-Grundkurses) sowie der Auswertung von Fragebögen (von 33 SchülerInnen) lassen sich als wichtigste Ergebnisse festhalten:

1. Bei einer 'Notenskala' von 0 bis 10 erhielten nur drei SchülerInnen eine Bewertung über 8,0 für ihre Exzerpte. Immerhin sieben SchülerInnen erhielten eine Bewertung zwischen 7,0 und 7,9. Knapp die Hälfte aller SchülerInnen (14) erhielt eine 'Note' im 'Mittelfeld' (zwischen 5,0 und 7,0). Vier Exzerpte wurden zwischen 4,0 und 4,9 bewertet, drei Exzerpte schlechter als 4,0. Zwei SchülerInnen hatten ihr Exzerpt nicht fertig gestellt.

Es ergab sich also ein differenziertes Bild, was die Qualität der einzelnen Exzerpte und damit die Exzerpierfähigkeit der SchülerInnen betrifft. Die wenigsten von ihnen erbrachten allerdings wirklich gute Leistungen. Aber selbst eine relativ gute Note (z. B. besser als 7,0) bedeutet nicht zwangsläufig, dass tatsächlich den Anforderungen des Studiums angemessene Exzerpierkenntnisse vorliegen; immerhin konnten selbst bei einer solchen "besseren" Bewertung noch einige Fehler gemacht werden. Es lässt sich anhand dieser Ergebnisse deshalb sagen, dass die meisten der an der Untersuchung beteiligten SchülerInnen ohne eine Vermittlung weiterer Kenntnisse vermutlich (große) Schwierigkeiten mit dem Exzerpieren von Texten im Studium hätten, z. B. von einschlägigen Artikeln für das Schreiben der ersten Hausarbeit. Sie wären also mit den hier festgestellten Exzerpierkenntnissen den entsprechenden Anforderungen im Studium nicht oder nicht in vollem Maße gewachsen.

2. Was die Ursachen für die Schwierigkeiten betrifft, so scheinen manche Fehler in den Exzerpten nahe zu legen, dass die SchülerInnen gelegentlich Probleme mit dem Textinhalt hatten (so ergab die Analyse der Exzerpte, dass eine bestimmte Primärtextstelle bei besonders vielen SchülerInnen zu Fehlern führte). Auch die Auswertung der Fragebögen zeigt, dass in gewissem Ausmaß Schwierigkeiten mit dem Text an sich vorhanden waren: dabei wurde der Textaufbau - im Gegensatz zur "Sprache" und "einzelnen Argumenten" des Textes - als am schwierigsten empfunden.

Der Großteil der Fehler kann jedoch nicht auf den Primärtext zurückgeführt werden. Dieser war so gewählt, dass ein Verständnis des Inhalts (zumindest größtenteils) gesichert war - dass dies tatsächlich der Fall war, legen die Schülerantworten auch nahe. So stufte beispielsweise keine/r von ihnen den Text als ganzen als "sehr schwierig" ein. Die meisten $(75,8 \%)$ empfanden ihn als "nicht sehr schwierig" bis "mittelschwierig". Generell scheinen die Ergebnisse vor allem der Fragebogenauswertung darauf hinzuweisen, dass weniger der Text selbst Probleme bereitete als vielmehr die unzureichenden Kenntnisse davon, wie gute Exzerpte anzufertigen sind.

Die Ergebnisse der Untersuchung zeigen also zum einen, dass die Exzerpierkenntnisse der SchülerInnen für die Anforderungen an der Universität nicht ausreichend sind (Punkt 1). Zwar ist das Exzerpieren von Texten eine Fähigkeit, die auch in der Schule (schon) durchaus nützlich wäre - insofern trifft Ehlichs (2000: 7) allgemeine Aussage auch für das Exzerpieren $\mathrm{zu}$, wenn er sagt, dass eine "Orientierung auf die Universität als ein schulisches Ausbildungsziel sinnvoll" sei. Doch hat die Untersuchung deutlich gezeigt, dass die Universitäten nicht 
davon ausgehen dürfen, dass eine solche Orientierung - was das Exzerpieren betrifft - auch tatsächlich in ausreichendem Maße erfolgt. Vielmehr ist davon auszugehen, "dass die spezifischen Fertigkeiten, die zum akademischen Schreiben befähigen, bei den StudienanfängerInnen nicht vorhanden sein können" (vgl. Dittmann et al. 2003: 163). Zu diesen Fertigkeiten gehört zweifellos das Anfertigen von Exzerpten.

Zum anderen ist das Ergebnis, wonach die Hauptursache der Schwierigkeiten nicht im Primärtext begründet lag (Punkt 2), ein starkes Argument dafür, das Exzerpieren an sich zu lehren, um den Problemen beizukommen. Auch Keselings (1993) Ergebnisse seiner Analyse von Zusammenfassungen legen diesen Schluss nahe: Er führt "Fehler" oder Verstöße beim Zusammenfassen auf eine unzureichende Kenntnis der abzulaufenden Prozeduren zurück (vgl. u. a. Keseling 1993: 137) - eben diese Prozeduren zum 'richtigen' Zusammenfassen von Texten aber müssten gelehrt werden. Derselbe Schluss ergibt sich aus dem von Dittmann et al. (2003) berichteten Ergebnis der Umfrage unter Studierenden, für die das Zusammenfassen der Fachliteratur ein viel größeres Problem darstellte als das Verstehen des Inhalts.

Die zwingende Konsequenz aus diesen Überlegungen ist aber, dass die Universitäten selbst ihre Studierenden mit den grundlegenden Techniken des Exzerpierens vertraut machen müssen. Da die Probleme der SchülerInnen (wie auch die der StudentInnen in der Umfrage von Dittmann et al. 2003) relativ unabhängig vom spezifischen Textinhalt zu bestehen scheinen, könnte das Lehren von Exzerpiertechniken durchaus fächerübergreifend erfolgen. Und auch wenn sich an der Lehrpraxis in den letzten 20 Jahren insofern nur wenig geändert hat als die Textart Exzerpt immer noch zu wenig Beachtung im Lehrangebot der Universitäten findet, so hat sich hier in anderer Hinsicht erfreulicherweise etwas verbessert.

Nachdem einige Erfahrungen in der Praxis (z. B. in Schreibberatungen) gesammelt sowie neue Erkenntnisse in der Textproduktionsforschung allgemein gewonnen wurden, kann man heute wohl sagen, dass Ehlichs Schluss von $1981 \mathrm{zu}$ pessimistisch ist, wonach eine Vermittlung der Textart Exzerpt an diejenigen SchülerInnen und Studierenden, die das Exzerpieren nicht sowieso schon beherrschen, nur schwer oder gar nicht möglich sei (vgl. Ehlich 1981: 382). So gibt es eine Reihe unterschiedlicher Methoden, den Lernenden das Exzerpieren zu vermitteln. Das von Kruse/Ruhmann (1999) vorgestellte Verfahren - "Aus Alt mach Neu: Vom Lesen zum Schreiben wissenschaftlicher Texte" - schildert beispielsweise eine ganz konkrete Vorgehensweise, mit der Studierenden (bzw. auch SchülerInnen) Schritt für Schritt die Technik des Exzerpierens beigebracht werden kann (weitere Beiträge hierzu finden sich im Sammelband Kruse/Jakobs/Ruhmann 1999). Eine "strenge schulische Übungsform" (Fritzsche 1994: 143), bei der ein Text auf ein Drittel seines ursprünglichen Umfangs verkürzt werden muss, ist beispielsweise der "Précis", den Fritzsche (1994) vorstellt. Dieser entspricht zwar nicht in allen Punkten einem Exzerpt, ist aber eine sinnvolle Technik, um SchülerInnen oder StudienanfängerInnen auf einfach zu vermittelnde Weise an das Zusammenfassen von Texten heranzuführen.

In erster Linie ist nun wichtig, dass überhaupt eine Vermittlung von Exzerpierkenntnissen an die Studierenden stattfindet. Bei Beratungsangeboten außerhalb der eigentlichen Lehrveranstaltungen (z. B. Schreibberatungen) ist allerdings zu beachten, dass eventuell nicht alle, die Rat nötig hätten, dort auch wirklich Hilfe suchen würden. Es muss deshalb ergänzend gefordert werden, die entsprechenden Kenntnisse innerhalb der regulären Lehre zu vermitteln. Dort nämlich, und diesen Aspekt darf man nicht vernachlässigen, kann auch der Relevanz der "Vorkenntnisse" Rechnung getragen werden, die sich bei der Erstellung von Exzerpten als wichtiger, die Qualität beeinflussender Faktor erwiesen haben (vgl. Abschnitt 4.6.2, Frage 11). Beide Lösungen erfordern als Grundvoraussetzung jedoch ein (größeres) Problembewusstsein bei den Lehrenden: Einerseits muss sinnvollen Einrichtungen wie Schreibberatungen größere Beachtung zugemessen werden, andererseits muss das Vermitteln von 
Exzerpierkenntnissen auch (und sogar wohl in erster Linie) innerhalb der Lehrveranstaltungen (z. B. in Einführungsveranstaltungen oder Tutoraten) erfolgen: Dem Exzerpieren sollte an der Universität endlich die Bedeutung zugemessen werden, die ihm tatsächlich zukommt.

\section{Literaturangaben}

Ballstaedt, Steffen-Peter (1999): "Textoptimierung. Von der Stilfibel zum Textdesign". Fachsprache 21: 98-124.

Becker-Mrotzek, Michael/Böttcher, Ingrid (2006): Schreibkompetenz. Entwickeln und beurteilen. Praxishandbuch für die Sekundarstufe I und II. Berlin.

Beste, Gisela (2003): "Schreibaufgaben im Deutschunterricht der Oberstufe. Vorbereitung auf die Hochschule?" In: Ehlich, Konrad/Steets, Angelika (eds.) (2003): Wissenschaftlich schreiben - lehren und lernen. Berlin/New York: 273-285.

Bünting, Karl-Dieter/Bitterlich, Axel/Pospiech, Ulrike (1996/2000): Schreiben im Studium. Ein Trainingsprogramm. 5. Aufl. Mit einem Beitrag von G. Ruhmann. Berlin.

Dijk, Teun A. van (1980): Textwissenschaft. Eine interdisziplinäre Einführung. Tübingen.

Dittmann, Jürgen et al. (2003): "Schreibprobleme im Studium. Eine empirische Untersuchung". In: Ehlich, Konrad/Steets, Angelika (eds.) (2003): Wissenschaftliche schreiben - lehren und lernen. Berlin/New York: 155-185.

Ehlich, Konrad (1981): "Zur Analyse der Textart 'Exzerpt'". In: Frier, Wolfgang (ed.) Pragmatik, Theorie und Praxis. Amsterdam: 379-401.

Ehlich, Konrad (2000): "Schreiben für die Hochschule". In: Ehlich, Konrad/Steets, Angelika/Traunspurger, Inka (eds.): Bibliographie "Schreiben für die Hochschule". Frankfurt am Main etc.: 1-17. (= Texproduktion und Medium 4).

Ehlich, Konrad/Steets, Angelika $\left(2000^{\mathrm{a}}\right)$ : "Schreiben im Studium". Einsichten. Forschung an der LMU 2/2000: 47-50.

Ehlich, Konrad/Steets, Angelika $\left(2000^{\mathrm{b}}\right)$ : Welche Rolle spielt das Schreiben im Rahmen der wissenschaftlichen Ausbildung? Ergebnisse einer fakultätsübergreifenden Umfrage an der LMU im Sommersemester 1999.

http://www.daf.uni-muenchen.de/DAF/FORSCHUNG/WISS_SCH/UMFRAGE.HTM, Stand 11.08.2001.

Endres-Niggemeyer, Brigitte/Schott, Hannelore (1992): "Ein individuelles prozedurales Modell des Abstracting". In: Krings, Hans P./Antos, Gerd (eds.): Textproduktion. Neue Wege der Forschung. Trier: 281-310.

Esselborn-Krumbiegel, Helga (2006): Leichter lernen. Strategien für Prüfung und Examen. Paderborn.

Fritzsche, Joachim (1994): Zur Didaktik und Methodik des Deutschunterrichts. Stuttgart:.

Furchner, Ingrid/Großmaß, Ruth/Ruhmann, Gabriela (1999): "Schreibberatung oder Studienberatung? Zwei Einrichtungen, zwei Zugangsweisen". In: Kruse, Otto/ Jakobs, Eva-Maria/Ruhmann, Gabriela (eds.): Schlüsselkompetenz Schreiben. Konzepte, Methoden, Projekte für Schreibberatung und Schreibdidaktik an der Hochschule. Neuwied/Kriftel: 37-60.

Furchner, Ingrid/Ruhmann, Gabriela/Tente, Christina $\left(1999^{\mathrm{b}}\right)$ : "Von der Schreibberatung für Studierende zur Lehrberatung für Dozenten". In: Kruse, Otto/Jakobs, Eva-Maria/Ruhmann, Gabriela (eds.): Schlüsselkompetenz Schreiben. Konzepte, Methoden, Projekte für Schreibberatung und Schreibdidaktik an der Hochschule. Neuwied/Kriftel: 61-72.

Gruber, Helmut et al. (2006): Genre, Habitus und wissenschaftliches Schreiben. Eine empirische Untersuchung studentischer Texte. Wien.

Jakobs, Eva-Maria (1997): "Lesen und Textproduzieren. Source reading als typisches Merkmal wissenschaftlicher Textproduktion". In: Jakobs, Eva-Maria/Knorr, Dagmar (eds.): Schreiben in den Wissenschaften. Frankfurt etc.: 75-90. 
Jakobs, Eva-Maria (1999): "Normen der Textgestaltung". In: Kruse, Otto/Jakobs, EvaMaria/Ruhmann, Gabriela (eds.): Schlüsselkompetenz Schreiben. Konzepte, Methoden, Projekte für Schreibberatung und Schreibdidaktik an der Hochschule. Neuwied/Kriftel: 171-190.

Johnson-Laird, Philip N. (1983): Mental Models. Towards a Cognitive Science of Language, Inference, and Consciousness. Cambridge.

Karmasin, Matthias/Ribing, Rainer (2006): Die Gestaltung wissenschaftlicher Arbeiten. Ein Leitfaden für Haus- und Seminararbeiten, Magisterarbeiten, Diplomarbeiten und Dissertationen. Wien.

Keseling, Gisbert (1993): Schreibprozeß und Textstruktur. Empirische Untersuchungen zur Produktion von Zusammenfassungen. Tübingen.

Kintsch, Walter/Dijk, Teun A. van (1983): Strategies of Discourse Comprehension. Orlando etc.

Kruse, Otto (ed.) (1998): Handbuch Studieren. Von der Einschreibung bis zum Examen. Frankfurt a. M./New York.

Kruse, Otto/Jakobs, Eva-Maria (1999): "Schreiben lehren an der Hochschule. Ein Überblick". In: Kruse, Otto/Jakobs, Eva-Maria/Ruhmann, Gabriela (eds.): Schlüsselkompetenz Schreiben. Konzepte, Methoden, Projekte für Schreibberatung und Schreibdidaktik an der Hochschule. Neuwied/Kriftel: 19-34.

Kruse, Otto/Jakobs, Eva-Maria/Ruhmann, Gabriela (eds.) (1999): Schlüsselkompetenz Schreiben. Konzepte, Methoden, Projekte für Schreibberatung und Schreibdidaktik an der Hochschule. Neuwied/Kriftel.

Kruse, Otto/Ruhmann, Gabriela (1999): "Aus Alt mach Neu. Vom Lesen zum Schreiben wissenschaftlicher Texte". In: Kruse, Otto/Jakobs, Eva-Maria/Ruhmann, Gabriela (eds.): Schlüsselkompetenz. Schreiben. Konzepte, Methoden, Projekte für Schreibberatung und Schreibdidaktik an der Hochschule. Neuwied/Kriftel: 109-121.

Ludwig, Otto (2003): "Entwicklungen der schulischen Schreibdidaktik und ihr Bezug zum akademischen Schreiben". In: Ehlich, Konrad/Steets, Angelika (eds.): Wissenschaftlich schreiben - lehren und lernen. Berlin/New York: 235-250.

Mann, William C./Thompson, Sandra A. (1988): "Rhetorical Structure Theory. Towards a functional theory of text organization". Text 8: 243-281.

Moll, Melanie (2002): "'Exzerpieren statt fotokopieren'. Das Exzerpt als zusammenfassende Verschriftlichung eines wissenschaftlichen Textes". In: Redder, Angelika (ed.): Effektiver studieren. Duisburg: 104-126. (= OBST-Beiheft 12).

Nussbaumer, Markus/Sieber, Peter (1994): "Texte analysieren mit dem Zürcher Textanalyseraster". In: Sieber, Peter (ed.): Sprachfähigkeit. Besser als ihr Ruf und nötiger denn je! Aarau etc.: 141-186.

Oldenburg, Hermann (1992): Angewandte Fachtextlinguistik. 'Conclusions' und Zusammenfassungen. Tübingen.

Rickheit, Gert/Strohner, Hans (1989): "Textreproduktion". In: Antos, Gerd/Krings, Hans P. (eds.): Textproduktion. Ein interdisziplinärer Forschungsüberblick. Tübingen: 220-256.

Rickheit, Gert/Strohner, Hans (1993): Grundlagen der kognitiven Sprachverarbeitung. Modelle, Methoden, Ergebnisse. Tübingen.

Rienecker, Lotte (1999): "Research questions and academic argumentation. Teaching students how to do it, using formats and model-examples". In: Kruse, Otto/Jakobs, EvaMaria/Ruhmann, Gabriela (eds.): Schlïsselkompetenz Schreiben. Konzepte, Methoden, Projekte für Schreibberatung und Schreibdidaktik an der Hochschule. Neuwied/Kriftel: 95-107.

Schnotz, Wolfgang (1988): "Textverstehen als Aufbau mentaler Modelle". In: Mandl, Heinz/Spada, Hans (eds.): Wissenspsychologie. Weinheim/Basel: 299-330. 
Stary, Joachim/Kretschmer, Horst (1999): Umgang mit wissenschaftlicher Literatur. Eine Arbeitshilfe für das sozial- und geisteswissenschaftliche Studium. Darmstadt.

Steets, Angelika (1999): "Schreiben in der Oberstufe. Überlegungen zu einer wissenschaftspropädeutischen Schreibausbildung". Mitteilungen des Deutschen Germanistenverbandes 46, 3: 398-421.

\section{Anhang 1: Primärtext und Aufgabenstellung}

1 10 eine Vertagung der Debatte notwendig.

Was war geschehen: Der Kölner Neurophysiologe Jürgen Hescheler hatte, fast nebenbei, von der Möglichkeit gesprochen, aus einem vier bis sieben Tage alten Embryo, der Blastozyste, Stammzellen zu gewinnen, ohne ihn dabei zu töten oder ihm zu schaden. „Wir werden dann keine Embryonen verbrauchen“, sagte Hescheler. Theoretisch gebe es dieses Verfahren, getestet aber sei es bisher nicht. Eine Stammzellgewinnung, die vergleichbar mit einer Knochenmarksspende ist, wäre die Lösung des großen ethischen Dilemmas von Forschung, Politik und Justiz. Gerhard Robbers vom Trierer Institut für Rechtspolitik hielt es für ein Gebot der Ethik, der Möglichkeit nachzugehen, auch für die DFG.

Solche Versuche aber sind Forschung an Embryonen und damit wie die Stammzell-Herstellung in Deutschland nach dem Embryonenschutzgesetz verboten. Dies wird sich auch mit der Entscheidung des Bundestags am 30.Januar nicht ändern, denn die Parlamentarier werden nur über den rechtlich zulässigen, aber ethisch umstrittenen Import embryonaler Stammzellen beschließen nach ihrem Gewissen. Vor allem SPD und CDU aber konnten keine Position ihrer Partei festlegen, die Politiker sind im Für und Wider ähnlich zerrissen wie die Juristen bei den Bitburger Gesprächen.

Die für die Forschung entscheidende Frage hat auch das Bundesverfassungsgericht bisher nicht 


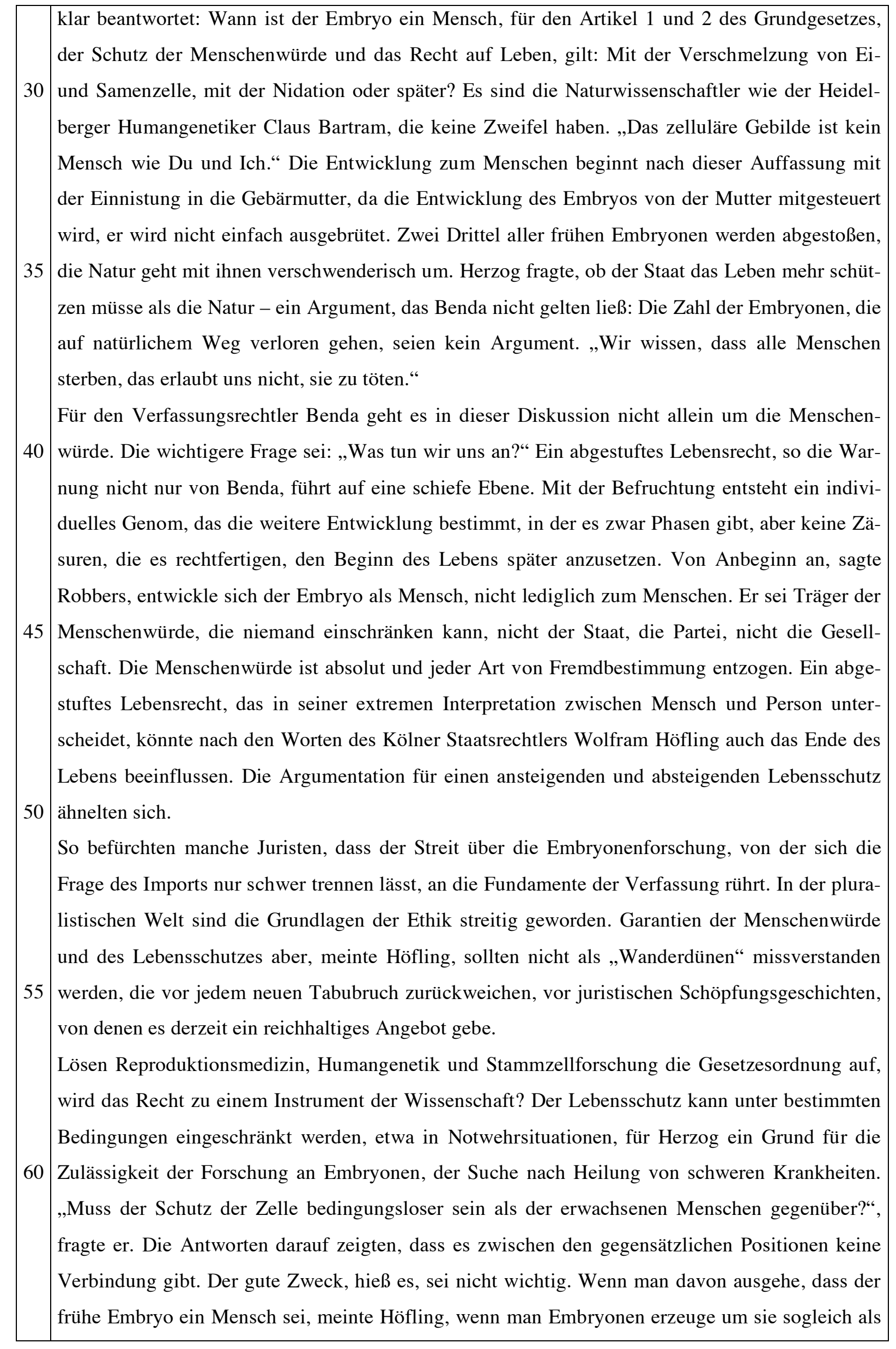


65 Forschungsmaterial zu verbrauchen und zu töten, „dann ist das monströs“. Und es mache keinen Unterschied, wenn es sich um „,̈̈berzählige Embryonen“ in der Petrischale handele, über deren Zahl gerätselt wird. Es gibt keine Statistik. Diese Embryonen würden, wie Höfling sagte, als Leichname qualifiziert, mit dem Argument, sie stürben ja ohnehin. „Todesnähe macht aus den Betroffenen keine Leichname.“

70 Der Vorwurf, unzählige Embryonen würden mit Verhütungsmitteln vernichtet, und bei der Abtreibung versage der Staat mit seiner Schutzpflicht, ist so alt wie die ganze Debatte. In die Privatsphäre aber kann der Staat nicht regelnd eingreifen, einen Vergleich mit der Abtreibung kann es nicht geben, die Konfliktsituation zwischen Mutter und Kind gilt als einzigartig.

$[\ldots]$

\section{Aufgabe:}

Verfasse ein Exzerpt des vorliegenden Textes, indem du die wichtigsten Aussagen und Argumente mit eigenen Worten zusammenfasst. (Stelle dir vor, das Exzerpt würde der Vorbereitung für ein Referat dienen, das du im Ethik-/Gemeinschaftskundeunterricht über dieses Thema halten müsstest.) 


\section{Anhang 2: Fragebogen 1}

Bei dieser Umfrage geht es darum herauszufinden, welche Rolle Exzerpte für euch als Schülerinnen und Schüler spielen. Ein Exzerpt ist die Zusammenfassung eines Textes und soll die wichtigsten Aussagen dieses ursprünglichen Textes beinhalten. Dabei ist der ursprüngliche Text länger und oft auch komplizierter als das daraus entstehende Exzerpt. Wenn jemand das Exzerpt liest, sollte er/sie die Gedanken und Argumente des Autors/der Autorin des ursprünglichen Textes nachvollziehen können auch wenn er/sie den ursprünglichen Text nicht gelesen hat.

1. Wurde mit dir im Schulunterricht schon einmal das Exzerpieren von Texten (z. B. Biologie- oder Deutschtexten) geübt?

ja In welchen Unterrichtsfächern (Mehrfachnennungen möglich)?

nein

weiß nicht

2. Hast du schon einmal - unabhängig davon, ob es im Schulunterricht schon geübt wurde oder nicht - einen Text exzerpiert?

ja nein

$\rightarrow$ Falls du die ersten beiden Fragen (Frage 1 und Frage 2) mit 'nein' beantwortet hast, kannst du direkt zu Frage 8 weiter.

3. Hast du dich schon einmal auf eine Klassenarbeit vorbereitet, indem Du einen wichtigen Text exzerpiert hast?

ja nein weiß nicht

4. Findest du, dass das Exzerpieren von Texten Dir hilft, diese besser zu verstehen?

ja nein weiß nicht

5. Hattest du schon einmal Probleme, einen Text zu exzerpieren (z. B. weil dieser zu kompliziert war)?

ja nein weiß nicht

$\rightarrow$ Falls 'ja', zu 6. - falls 'nein', weiter zu 8.

6. Wie oft hast Du Probleme beim Exzerpieren von Texten?

sehr oft oft manchmal selten

7. Als wie schlimm empfindest du diese Probleme? Bitte kreuze in der folgenden Skala an:

$\begin{array}{lllllll}\text { überhaupt nicht schlimm }(=1) & 1 & 2 & 3 & 4 & 5 & \operatorname{sehr} \operatorname{schlimm}(=5)\end{array}$

8. Im Folgenden ist eine Aussage gegeben. Bitte bewerte diese in einer Skala von 1 (= 'stimme überhaupt nicht zu') bis 5 (= 'stimme voll zu'); (Alternative: 'weiß nicht'). 
Ich halte das Exzerpieren von Fachtexten (z. B. Biologie, Geschichte etc.) für eine gute Möglichkeit, sich auf Klassenarbeiten vorzubereiten.

stimme überhaupt nicht zu (=1) $\quad \begin{array}{lllllll} & 1 & 2 & 3 & 4 & 5 & \text { stimme voll zu (=5) }\end{array}$ weiß nicht

9. Möchtest du später einmal studieren?

ja nein weiß ich jetzt noch nicht

$\rightarrow$ Falls 'ja' oder 'weiß noch nicht', zu 10. - falls 'nein', weiter zu 11.

10. Weißt du schon, was bzw. welche Fachrichtung Du einmal studieren möchtest?

ja: (Fach bzw. Fachrichtung)

nein, weiß ich noch nicht

Persönliche Angaben:

11. Alter: Jahre

12. Geschlecht: m

W

13. Leistungskurse: und

\section{Vielen Dank für deine Mitarbeit.}




\section{Anhang 3: Fragebogen 2}

1. Wie schwierig fandest du den Text, den du exzerpiert hast? Bitte ordne inn in einer Schwierigkeitsskala von 1 bis 5 ein.

$\begin{array}{lllllll}\text { überhaupt nicht schwierig (=1) } & 1 & 2 & 3 & 4 & 5 & \text { sehr schwierig (=5) }\end{array}$

In den Fragen 2 bis 10 sind (kursiv) Aussagen gegeben. Bitte bewerte diese auf einer Skala von 1 (= stimme überhaupt nicht zu) bis 5 (= stimme voll zu).

2. Einmaliges Lesen reichte aus, um den Text vollständig zu verstehen.

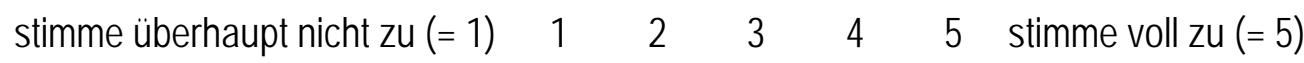

3. Ich fand es schwierig, diesen Text zu exzerpieren.

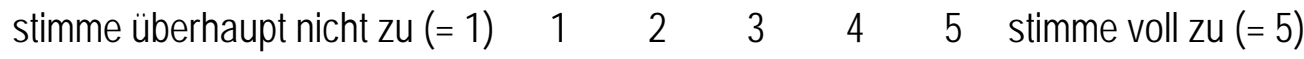

4. Die Sprache/Ausdrucksweise des Texts war schwer verständlich.

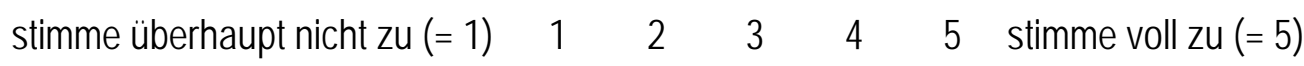

5. Die Argumente der Autorin waren schwer zu verstehen.

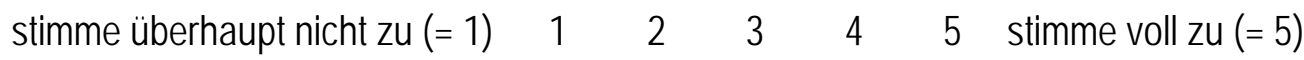

6. Der Textaufbau war schwer nachvollziehbar (= die Art und Weise, wie die Argumente aufeinander folgten oder auseinander heraus entwickelt wurden).

stimme überhaupt nicht zu (= 1) $\quad \begin{array}{lllllll}1 & 2 & 3 & 4 & 5 & \text { stimme voll zu (= 5) }\end{array}$

7. Es fiel mir schwer, den Text zu kürzen.

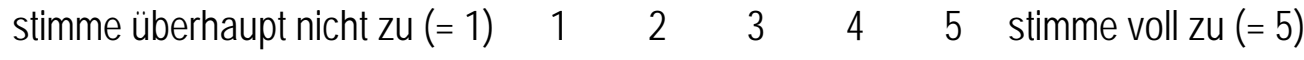

8. Es fiel mir schwer, einzelne Argumente des Textes herauszuarbeiten.

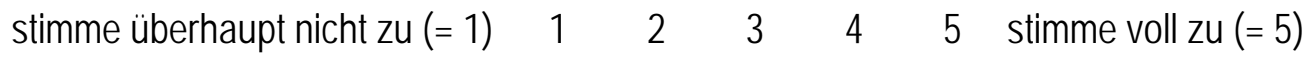

9. Das Exzerpieren hat mir zu einem besseren Textverständnis verholfen als zuvor reines Lesen des Textes.

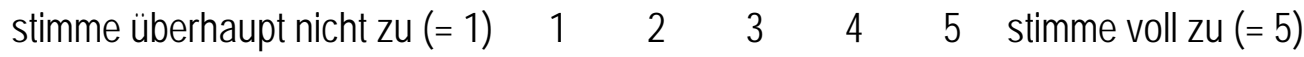

10. Durch meinen (Deutsch-) Unterricht habe ich mich für diese Aufgabe ausreichend vorbereitet gefühlt.

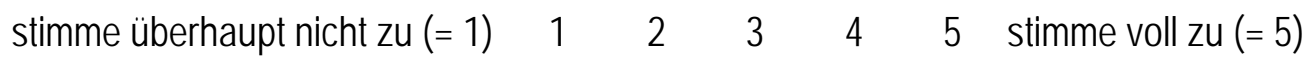

11. Hattest du Vorkenntnisse im Themengebiet des Textes (z. B. Import embryonaler Stammzellen)? ja nein ein bisschen 
12. Falls du Probleme beim Erstellen des Exzerpts hattest (z. B. Zeitdruck, Text zu schwierig etc.), könntest du diese bitte kurz erläutern?

$\rightarrow$ Antwort bitte auf die Rückseite!

Allgemeine Fragen:

13. Hältst du das Exzerpieren allgemein für eine bedeutsame Arbeitsmethode für dich als Schüler/Schülerin? Bitte kreuze in der folgenden Skala an:

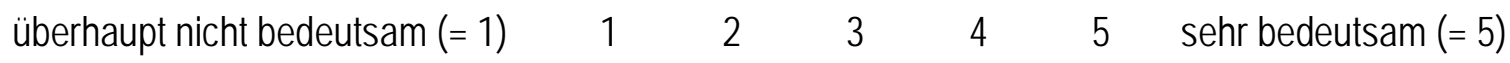

14. Falls überhaupt, inwiefern ist das Exzerpieren für dich von Bedeutung (z. B. Vorbereitung auf das Abitur)?

Vielen Dank für deine Mitarbeit. 
Anhang 4: Makropropositionen des Primärtextes

\begin{tabular}{|c|c|c|}
\hline 1. Makroebene & 2. Makroebene & 3. Makroebene \\
\hline $\begin{array}{l}\text { M1;1: 40. Bitburger Gespräche } \\
\text { zum Thema "Der entschlüsselte } \\
\text { Mensch" fanden statt }\end{array}$ & $\begin{array}{l}\text { M2;1: 40. Bitburger Gespräche } \\
\text { zum Thema "Der entschlüsselte } \\
\text { Mensch" fanden statt }\end{array}$ & \\
\hline $\begin{array}{l}\text { M1;2: Es herrschte allgemeine } \\
\text { Aufregung wegen M1;7 }\end{array}$ & $\begin{array}{l}\text { M2;2: M2;3 sorgte bei den } \\
\text { Beteiligten für Aufregung }\end{array}$ & \\
\hline $\begin{array}{l}\text { M1;3: H. von Kalm erachtete } \\
\text { M1;7 als wichtig }\end{array}$ & M2;2 & \\
\hline $\begin{array}{l}\text { M1;4: R. Herzog erachtete M1;7 } \\
\text { als wichtig }\end{array}$ & $\mathrm{M} 2 ; 2$ & \\
\hline $\begin{array}{l}\text { M1;5: E. Benda erachtete M1;7 als } \\
\text { wichtig }\end{array}$ & $\mathrm{M} 2 ; 2$ & \\
\hline $\begin{array}{l}\text { M1;6: Der Kölner Neurophysio- } \\
\text { loge J. Hescheler gab M1;7 } \\
\text { bekannt }\end{array}$ & M2;3 & \\
\hline $\begin{array}{l}\text { M1;7: Es gibt theoretisch die } \\
\text { Möglichkeit, aus einem vier bis } \\
\text { sieben Tage alten Embryo } \\
\text { Stammzellen zu gewinnen, ohne } \\
\text { ihm dabei zu schaden }\end{array}$ & $\begin{array}{l}\text { M2;3: Es gibt theoretisch die } \\
\text { Möglichkeit, aus einem vier bis } \\
\text { sieben Tage alten Embryo } \\
\text { Stammzellen zu gewinnen, ohne } \\
\text { ihm dabei zu schaden }\end{array}$ & $\begin{array}{l}\text { M3;1: Es gibt einen theoretischen } \\
\text { Ausweg für das Dilemma (M3;3) }\end{array}$ \\
\hline $\begin{array}{l}\text { M1;8: Verfahren }(\mathrm{M} 1 ; 7) \text { ist noch } \\
\text { nicht getestet }\end{array}$ & $\begin{array}{l}\text { M2;4: Verfahren }(\mathrm{M} 2 ; 3) \text { ist noch } \\
\text { nicht getestet }\end{array}$ & $\begin{array}{l}\text { M3;2: Allerdings kann M3;3 nur } \\
\text { erprobt werden, falls das } \\
\text { Embryonenschutzgesetz in } \\
\text { Deutschland geändert wird } \\
\end{array}$ \\
\hline $\begin{array}{l}\text { M1;9: Falls M1;7 möglich ist, } \\
\text { wäre dies (nach Ansicht der } \\
\text { Autorin) die Lösung des } \\
\text { "ethischen Dilemmas von } \\
\text { Forschung, Politik und Justiz" }\end{array}$ & $\begin{array}{l}\text { M2;5: Falls M2;3 möglich ist, } \\
\text { wäre dies die Lösung des } \\
\text { "ethischen Dilemmas von } \\
\text { Forschung, Politik und Justiz" }\end{array}$ & \\
\hline \multicolumn{3}{|l|}{$\begin{array}{l}\text { M1;10: G. Robbers hielt es für } \\
\text { wichtig, M1;7 zu testen }\end{array}$} \\
\hline $\begin{array}{l}\text { M1;11: Auf Grund des } \\
\text { Embryonenschutzgesetzes ist es in } \\
\text { Deutschland verboten, } \mathrm{M} 1 ; 7 \mathrm{zu} \\
\text { testen }\end{array}$ & $\begin{array}{l}\text { M2;6: Auf Grund des } \\
\text { Embryonenschutzgesetzes ist es in } \\
\text { Deutschland verboten, M2;3 zu } \\
\text { testen }\end{array}$ & \\
\hline \multicolumn{3}{|l|}{$\begin{array}{l}\text { M1;12: Auch M1;13 wird M1;11 } \\
\text { nicht ändern }\end{array}$} \\
\hline \multicolumn{3}{|l|}{$\begin{array}{l}\text { M1;13: Entscheidung des } \\
\text { Bundestages am 30. Januar über } \\
\text { den ethisch umstrittenen Import } \\
\text { embryonaler Stammzellen }\end{array}$} \\
\hline $\begin{array}{l}\text { M1;14: Die Politiker sind sich } \\
\text { nicht einig, was M1;13 betrifft } \\
\text { (selbst innerparteilich nicht) }\end{array}$ & $\begin{array}{l}\text { M2;7: Sowoh1 Politiker als auch } \\
\text { Juristen sind zerrissen, was die } \\
\text { Forschung mit embryonalen } \\
\text { Stammzellen betrifft }\end{array}$ & \\
\hline $\begin{array}{l}\text { M1;15: Die Juristen sind sich nicht } \\
\text { einig, was die Forschung mit } \\
\text { embryonalen Stammzellen betrifft }\end{array}$ & M2;7 & \\
\hline $\begin{array}{l}\text { M1;16: Unabhängig von M1;1- } \\
\text { 1;15 ist die Beantwortung von } \\
\text { M1;17 für die Forschung } \\
\text { entscheidend }\end{array}$ & $\begin{array}{l}\text { M2;8: Unabhängig von M2;1- } \\
\text { M2;6 ist die Beantwortung von } \\
\text { M2;9 für die Forschung } \\
\text { entscheidend }\end{array}$ & $\begin{array}{l}\text { M3;3: Politiker, Juristen und } \\
\text { Forscher (bei den Bitburgern } \\
\text { Gesprächen) sind sich nicht einig } \\
\text { darüber, ob man Embryonen für } \\
\text { Forschungszwecke töten dürfe } \\
\end{array}$ \\
\hline $\begin{array}{l}\text { M1;17: Es ist fraglich, wann der } \\
\text { Embryo ein Mensch ist }\end{array}$ & $\begin{array}{l}\text { M2;9: Es ist fraglich, wann der } \\
\text { Embryo ein Mensch ist }\end{array}$ & \\
\hline
\end{tabular}




\begin{tabular}{|c|c|c|}
\hline $\begin{array}{l}\text { M1;18: Ein Mensch hat den } \\
\text { Schutz der Menschenwürde und } \\
\text { das Recht auf Leben }\end{array}$ & $\begin{array}{l}\text { M2;10: Ein Mensch hat den } \\
\text { Schutz der Menschenwürde und } \\
\text { das Recht auf Leben }\end{array}$ & \\
\hline $\begin{array}{l}\text { M1;19: Es ist fraglich, ob für die } \\
\text { Beantwortung von M1;17 die } \\
\text { Verschmelzung von Ei- und } \\
\text { Samenzelle, die Nidation oder ein } \\
\text { späterer Zeitpunkt in der } \\
\text { Entwicklung gilt }\end{array}$ & M2;9 & \\
\hline $\begin{array}{l}\text { M1;20: Naturwissenschaftler wie } \\
\text { der Humangenetiker C. Bartram } \\
\text { haben Auffassung M1;21 }\end{array}$ & M2;11 & \\
\hline $\begin{array}{l}\text { M1;21: Auffassung, nach der die } \\
\text { Entwicklung des Embryos zum } \\
\text { Menschen mit der Einnistung in } \\
\text { die Gebärmutter beginnt }\end{array}$ & $\begin{array}{l}\text { M2;11: Befürworter der ES- } \\
\text { Forschung haben die Auffassung, } \\
\text { dass die Entwicklung des Embryos } \\
\text { zum Menschen erst mit der } \\
\text { Einnistung in die Gebärmutter } \\
\text { beginnt }\end{array}$ & $\begin{array}{l}\text { M3;4: Befürworter der ES- } \\
\text { Forschung finden, dass man 'frühe' } \\
\text { Embryonen (vor der Einnistung in } \\
\text { die Gebärmutter) für } \\
\text { Forschungszwecke verwenden } \\
\text { dürfe } \\
\end{array}$ \\
\hline $\begin{array}{l}\text { M1;22: M1;21, da die } \\
\text { Entwicklung des Embryos von der } \\
\text { Mutter abhängig ist }\end{array}$ & $\begin{array}{l}\text { M2;12: M2;11, da die } \\
\text { Entwicklung des Embryos von der } \\
\text { Mutter abhängig ist }\end{array}$ & $\begin{array}{l}\text { M3;5: M3;4, da der Embryo erst } \\
\text { mit der Einnistung in die } \\
\text { Gebärmutter ein Mensch sei }\end{array}$ \\
\hline $\begin{array}{l}\text { M1;23: Zwei Drittel aller frühen } \\
\text { Embryonen gehen auf natürliche } \\
\text { Weise verloren }\end{array}$ & $\begin{array}{l}\text { M2;13: Viele frühe Embryonen } \\
\text { gehen auf natürliche Weise } \\
\text { verloren }\end{array}$ & \\
\hline $\begin{array}{l}\text { M1;24: R. Herzog war der } \\
\text { Ansicht, dass der Staat das Leben } \\
\text { nicht mehr schützen müsse als die } \\
\text { Natur }\end{array}$ & $\begin{array}{l}\text { M2;14: Befürworter nehmen } \\
\text { M2;13 als Argument für die } \\
\text { Embryonenforschung }\end{array}$ & \\
\hline $\begin{array}{l}\text { M1;25: E. Benda war der Ansicht, } \\
\text { M1;23 gelte nicht als Argument }\end{array}$ & $\begin{array}{l}\text { M2;15: Gegner der ES-Forschung } \\
\text { lassen M2;13 als Argument nicht } \\
\text { gelten }\end{array}$ & \\
\hline \multicolumn{3}{|l|}{$\begin{array}{l}\text { M1;26: M1;25, da das Wissen um } \\
\text { die Sterblichkeit der Menschen } \\
\text { keine Tötungserlaubnis liefere }\end{array}$} \\
\hline $\begin{array}{l}\text { M1;27: E. Benda und andere } \\
\text { waren der Ansicht, dass ein } \\
\text { abgestuftes Lebensrecht nicht } \\
\text { rechtfertigt sei }\end{array}$ & $\begin{array}{l}\text { M2;16: Gegner der ES-Forschung } \\
\text { sind der Ansicht, dass der Embryo } \\
\text { von Anfang an das Recht auf } \\
\text { Leben habe }\end{array}$ & $\begin{array}{l}\text { M3;6: Gegner sind der Ansicht, } \\
\text { dass auch frühe Embryonen nicht } \\
\text { für die Forschung verwendet } \\
\text { werden dürfen }\end{array}$ \\
\hline $\begin{array}{l}\text { M1;28: M1;27, da bereits bei der } \\
\text { Befruchtung das Genom entsteht, } \\
\text { das die weitere Entwicklung } \\
\text { bestimmt }\end{array}$ & $\begin{array}{l}\text { M2;17: M2;16, da ein abgestuftes } \\
\text { Lebensrecht durch die } \\
\text { Entwicklung des Embryos nicht } \\
\text { gerechtfertigt sei }\end{array}$ & $\begin{array}{l}\text { M3;7: M6, da die Entwicklung des } \\
\text { Embryos kein abgestuftes } \\
\text { Lebensrecht rechtfertige }\end{array}$ \\
\hline $\begin{array}{l}\text { M1;29: M1;27, da es in der } \\
\text { Entwicklung des Embryos zwar } \\
\text { Phasen gibt, aber keine Zäsuren }\end{array}$ & M2;17 & \\
\hline $\begin{array}{l}\text { M1;30: Auch G. Robbers vertrat } \\
\text { Auffassung M1;27 }\end{array}$ & M2;16 & \\
\hline $\begin{array}{l}\text { M1;31: G. Robbers war der } \\
\text { Ansicht, dass niemand die } \\
\text { Menschenwürde einschränken } \\
\text { kann }\end{array}$ & M2;20 & \\
\hline $\begin{array}{l}\text { M1;32: Ein abgestuftes } \\
\text { Lebensrecht könne sich - so W. } \\
\text { Höfling - auch auf den } \\
\text { Lebensschutz am Ende des Lebens } \\
\text { auswirken }\end{array}$ & $\begin{array}{l}\text { M2;18: Gegner warnen, dass ein } \\
\text { abgestuftes Lebensrecht sich auch } \\
\text { auf den Lebensschutz am Ende des } \\
\text { Lebens auswirken kann }\end{array}$ & \\
\hline $\begin{array}{l}\text { M1;33: Manche Juristen } \\
\text { befürchten, der Streit über die } \\
\text { Embryonenforschung rühre an die } \\
\text { Fundamente der Verfassung }\end{array}$ & $\begin{array}{l}\text { M2;19: Manche Juristen } \\
\text { befürchten, der Streit über die } \\
\text { Embryonenforschung rühre an die } \\
\text { Fundamente der Verfassung }\end{array}$ & \\
\hline
\end{tabular}




\begin{tabular}{|c|c|c|}
\hline $\begin{array}{l}\text { M1;34: W. Höfling war der } \\
\text { Ansicht, dass die Menschenwürde } \\
\text { und der Lebensschutz absolut } \\
\text { garantiert sein müssen }\end{array}$ & $\begin{array}{l}\text { M2;20: Befürworter sind der } \\
\text { Ansicht, dass die Menschenwürde } \\
\text { und der Lebensschutz absolut } \\
\text { garantiert sein müssen }\end{array}$ & \\
\hline $\begin{array}{l}\text { M1;35: Unter bestimmten } \\
\text { Bedingungen kann der } \\
\text { Lebensschutz (auch für } \\
\text { Erwachsene) eingeschränkt } \\
\text { werden }\end{array}$ & $\begin{array}{l}\text { M2;21: Unter bestimmten } \\
\text { Bedingungen kann der } \\
\text { Lebensschutz (auch für } \\
\text { Erwachsene) eingeschränkt } \\
\text { werden }\end{array}$ & \\
\hline $\begin{array}{l}\text { M1;36: Durch } \\
\text { Embryonenforschung kann man } \\
\text { Heilung für schwere Krankheiten } \\
\text { finden }\end{array}$ & $\begin{array}{l}\text { M2;22: Durch } \\
\text { Embryonenforschung kann man } \\
\text { Heilung für schwere Krankheiten } \\
\text { finden }\end{array}$ & $\begin{array}{l}\text { M3;8: M3;4, da man durch die } \\
\text { Forschung Heilung für schwere } \\
\text { Krankheiten finden könne }\end{array}$ \\
\hline $\begin{array}{l}\text { M1;37: R. Herzog war der } \\
\text { Ansicht, dass wegen M1;35 und } \\
\text { M1;36 die Forschung an } \\
\text { Embryonen gerechtfertigt ist }\end{array}$ & $\begin{array}{l}\text { M2;23: Befürworter (z. B. R. } \\
\text { Herzog) ziehen M2;21 und M2;22 } \\
\text { als Argumente für die } \\
\text { Embryonenforschung heran }\end{array}$ & \\
\hline $\begin{array}{l}\text { M1;38: Gegner der } \\
\text { Embryonenforschung sind der } \\
\text { Ansicht, M1;36 (der gute Zweck) } \\
\text { sei nicht wichtig }\end{array}$ & M2;24 & \\
\hline $\begin{array}{l}\text { M1;39: Es sei falsch, so die } \\
\text { Gegner, Embryonen nur zu } \\
\text { Forschungszwecken zu erzeugen } \\
\text { und dann zu töten }\end{array}$ & $\begin{array}{l}\text { M2;24: Gegner sagen, es sei } \\
\text { grundsätzlich falsch, Embryonen } \\
\text { (zu Forschungszwecken) zu töten }\end{array}$ & $\begin{array}{l}\text { M3;9: M3;6, da der gute Zweck } \\
\text { nicht wichtig sei, das Töten von } \\
\text { Embryonen also grundsätzlich } \\
\text { falsch sei }\end{array}$ \\
\hline $\begin{array}{l}\text { M1;40: Es sei auch falsch, so die } \\
\text { Gegner, schon vorhandene } \\
\text { Embryonen für Forschungszwecke } \\
\text { zu töten }\end{array}$ & M2;24 & \\
\hline $\begin{array}{l}\text { M1;41: M1;40, da man die Tötung } \\
\text { von Embryonen nicht damit } \\
\text { rechtfertigen darf, dass sie ohnehin } \\
\text { stürben }\end{array}$ & M2;24 & \\
\hline $\begin{array}{l}\text { M1;42: Viele Embryonen werden } \\
\text { mit Verhütungsmitteln vernichtet }\end{array}$ & M2;25 & \\
\hline $\begin{array}{l}\text { M1;43: Viele Embryonen werden } \\
\text { durch Abtreibung vernichtet }\end{array}$ & M2;25 & \\
\hline $\begin{array}{l}\text { M1;44: M1;42 und M1;43 werden } \\
\text { als Argumente für die } \\
\text { Embryonenforschung } \\
\text { herangezogen }\end{array}$ & M2;25 & \\
\hline $\begin{array}{l}\text { M1;45: Der Staat kann in die } \\
\text { Privatsphäre nicht eingreifen }\end{array}$ & M2;25 & \\
\hline $\begin{array}{l}\text { M1;46: Die Konfliktsituation bei } \\
\text { der Abtreibung gilt als einzigartig }\end{array}$ & M2;25 & \\
\hline $\begin{array}{l}\text { M1;47: Wegen M1;45 und M1;46 } \\
\text { ist M1;44 nicht sinnvoll }\end{array}$ & $\begin{array}{l}\text { M2;25: Vergleiche mit Verhütung } \\
\text { oder Abtreibung (wie sie oft } \\
\text { gemacht wurden) sind in diesem } \\
\text { Zusammenhang nicht sinnvoll }\end{array}$ & \\
\hline
\end{tabular}




\section{Anhang 5: Ein "ideales Exzerpt"}

Bei den 40. Bitburger Gesprächen zum Thema "Der entschlüsselte Mensch" hatte der Kölner Neurophysiologe J. Hescheler erstmals von der Möglichkeit gesprochen, aus einem vier bis sieben Tage alten Embryo Stammzellen zu gewinnen, ohne ihm dabei zu schaden. Dies sorgte bei den Beteiligten für große Aufregung, da solch eine Art der Stammzellgewinnung die Lösung "des großen ethischen Dilemmas von Forschung, Politik und Justiz" wäre.

Dieses theoretisch mögliche Verfahren zu testen ist in Deutschland aber auf Grund des Embryonenschutzgesetzes verboten.

Unabhängig von dieser neuen Methode sind sich Politiker, Juristen und Forscher nicht einig, was die für die Forschung entscheidende Frage betrifft, ab wann in seiner Entwicklung der Embryo nämlich ein Mensch sei und damit das Recht auf Leben habe.

Befürworter der ES-Forschung (z. B. der Humangenetiker C. Bartram) vertreten die Auffassung, die Entwicklung des Embryos zum Menschen beginne erst mit der Einnistung in die Gebärmutter, da seine Entwicklung von der Mutter mitgesteuert werde. Auch die Tatsache, dass viele frühe Embryonen auf natürliche Weise abgestoßen werden, wird als Argument für die ES-Forschung benutzt (z. B. von R. Herzog).

Die Gegner lassen solch ein Argument nicht gelten und betonen, dass der Embryo von Anfang an das Recht auf Leben habe; ein abgestuftes Lebensrecht sei durch die Entwicklung des Embryos nämlich nicht rechtfertigt.

Wenn sich - was den Beginn der embryonalen Entwicklung betrifft - ein abgestuftes Lebensrecht durchsetzt, könne dies nach Ansicht des Kölner Staatsrechtlers W. Höfling auch das Ende des Lebens beeinflussen. Manche Juristen befürchten sogar, dass der Streit über die Embryonenforschung "an die Fundamente der Verfassung" rühre. Befürworter (z. B. W. Höfling) betonen deshalb, dass die Menschenwürde und der Lebensschutz absolut garantiert sein müssen.

Dass nun selbst beim erwachsenen Menschen der Lebensschutz in bestimmten Situationen eingeschränkt werden kann (z. B. Notwehr), rechtfertigt für Befürworter wie R. Herzog die Embryonenforschung. Zudem sei sie bedeutend bei der Suche nach Heilung von schweren Krankheiten.

Für die Gegner wiederum (z. B. W. Höfling) ist das Töten von Embryonen für die Forschung grundsätzlich falsch.

Oft gemachte Vergleiche mit der Verhütung oder Abtreibung (bei denen der Staat mit seiner Schutzpflicht versage) erscheinen in diesem Zusammenhang nicht sinnvoll. 